Pure and Applied Mathematics Quarterly

Volume 2, Number 1

(Special Issue: In honor of

John H. Coates, Part 1 of 2$)$

$29-110,2006$

\title{
Modular Curves and Rigid-Analytic Spaces
}

\author{
Brian Conrad
}

\section{Contents}

1. Introduction 30

1.1. Motivation 30

1.2. Overview of results 30

1.3. Notation and terminology 32

2. Analytic generalized elliptic curves 33

2.1. Basic definitions over a rigid-analytic space 33

2.2. Global structure of generalized elliptic curves over rigid spaces 41

3. Universal structures 52

3.1. Relative rigid Tate curves and a variant on Berthelot's functor $\quad 52$

3.2. Degenerate fibers and modular curves 58

4. Canonical subgroups 69

4.1. The $p$-torsion case $\quad 69$

4.2. Higher torsion-level canonical subgroups $\quad 75$

4.3. Frobenius lifts 81

Appendix A. Some properties of morphisms $\quad 86$

Received July 23, 2005.

1991 Mathematics Subject Classification. Primary 14G22; Secondary 14H52.

This research was partially supported by NSF grant DMS-0093542, the Alfred P. Sloan Foundation, and the Clay Mathematics Institute. I would like to thank L. Lipshitz and M. Temkin for helpful discussions, and K. Buzzard for sustained encouragement while this circle of ideas evolved. 
A.1. Properties of finite morphisms $\quad 86$

A.2. Change of base field 90

Appendix B. Algebraic theory of generalized elliptic curves 101

B.1. Deligne-Rapoport semistable curves of genus 1

$\begin{array}{lll}\text { B.2. } & \text { Generalized elliptic curves over schemes } & 103\end{array}$

$\begin{array}{ll}\text { B.3. Reduction-type and formal canonical subgroups } & 106\end{array}$

$\begin{array}{ll}\text { References } & 109\end{array}$

\section{INTRODUCTION}

1.1. Motivation. In the original work of Katz on $p$-adic modular forms $[\mathrm{Kz}]$, a key insight is the use of Lubin's work on canonical subgroups in 1-parameter formal groups to define a relative theory of a "canonical subgroup" in $p$-adic families of elliptic curves whose reduction types are good but not too supersingular. The theory initiated by Katz has been refined in various directions (as in [AG], $[\mathrm{AM}],[\mathrm{Bu}],[\mathrm{GK}],[\mathrm{G}],[\mathrm{KL}])$. The philosophy emphasized in this paper and its sequel [C4] in the higher-dimensional case is that by making fuller use of techniques in rigid geometry, the definitions and results in the theory can be made applicable to families over rather general rigid-analytic spaces over arbitrary analytic extensions $k / \mathbf{Q}_{p}$ (including base fields such as $\mathbf{C}_{p}$, for which Galois-theoretic techniques as in $[\mathrm{AM}]$ are not applicable).

The aim of this paper is to give a purely rigid-analytic development of the theory of canonical subgroups with arbitrary torsion-level in generalized elliptic curves over rigid spaces over $k$ (using Lubin's $p$-torsion theory only over valuation rings, which is to say on fibers), with an eye toward the development of a general theory of canonical subgroups in $p$-adic analytic families of abelian varieties that we shall discuss in [C4]. An essential feature is to work with rigid spaces and not with formal models in the fundamental definitions and theorems, and to avoid unnecessary reliance on the fine structure of integral models of modular curves. The 1-dimensional case exhibits special features (such as moduli-theoretic compactification and formal groups in one rather than several parameters) and it is technically simpler, so the results in this case are more precise than seems possible in the higher-dimensional case. It is therefore worthwhile to give a separate treatement in the case of relative dimension 1 as we do in the present paper.

1.2. Overview of results. The starting point for our work is [C3], where we develop a rigid-analytic theory of relative ampleness for line bundles and a rigidanalytic theory of fpqc descent for both morphisms and proper geometric objects 
equipped with a relatively ample line bundle. (For coherent sheaves, the theory of fpqc descent was worked out by Bosch and Görtz [BG].) In $§ 2.1-\S 2.2$ we use [C3] to construct a global theory of generalized elliptic curves over arbitrary rigidanalytic spaces. Without a doubt, the central difficulty is to establish finiteness properties for torsion, such as finiteness of the morphism $[d]: E^{\mathrm{sm}} \rightarrow E^{\mathrm{sm}}$ when $E \rightarrow S$ is a rigid-analytic generalized elliptic curve (with relative smooth locus $\left.E^{\mathrm{sm}}\right)$ such that the non-smooth fibers are geometrically $d$-gons. Such finiteness properties are used as input in the proof of "algebraicity" locally on the base for any rigid-analytic generalized elliptic curve $E \rightarrow S$, as well as algebraicity for the action map on $E$ by the $S$-group $E^{\mathrm{sm}}$ that is generally not quasi-compact over $S$. The main ingredient in these arguments is the compatibility of representing objects for algebraic and rigid-analytic Hilbert and Hom functors.

To illustrate the general theory, in $\S 3.1$ we discuss relative Tate curves from a viewpoint that applies to higher-dimensional moduli spaces for which reasonable proper integral models are available. In $\S 3.2$ we prove rigid-analytic universality of analytified algebraic modular curves (the point being to include the cusps on an equal footing). Our arguments are applied in the higher-dimensional setting in [C4], and so we use methods that may appear to be more abstract than necessary for the case of relative dimension 1 .

In $\S 4.1$ these results are applied over general analytic extension fields $k / \mathbf{Q}_{p}$ to develop a relative rigid-analytic theory of the canonical subgroup of Katz and Lubin. The fine structure of modular curves does not play as dominant a role as in other treatments of the theory, and the key to our method is a fibral criterion (Theorem A.1.2) for a quasi-compact, flat, and separated map of rigid spaces to be finite. Canonical subgroups with higher torsion level are treated in $\S 4.2$, where we show that our non-inductive definition for canonical subgroups is equivalent to the inductive one used in [Bu]; in particular, an ad hoc hypothesis on Hasse invariants in the inductive definition in $[\mathrm{Bu}]$ is proved to be necessary and sufficient for existence under the non-inductive definition. We conclude in $\S 4.3$ by relating level- $n$ canonical subgroups to characteristic-0 lifts of the kernel of the $n$-fold relative Frobenius map modulo $p^{1-\varepsilon}$ for arbitrarily small $\varepsilon>0$.

In Appendix A we review some background results in rigid geometry for which we do not know references. In Appendix B we review results of Deligne-Rapoport and Lubin that are used in our work with generalized elliptic curves in the rigidanalytic case.

We suggest that the reader look at the definitions in $\S 2.1$ and then immediately skip ahead to $\S 4$, only going back to the foundational $\S 2-\S 3$ as the need arises (in proofs, examples, and so on). 
1.3. Notation and terminology. If $S$ is a scheme, we sometimes write $X_{/ S}$ to denote an $S$-scheme and $X_{/ S^{\prime}}$ to denote the base change $X \times_{S} S^{\prime}$ over an $S$ scheme $S^{\prime}$. If $X \rightarrow S$ is a flat morphism locally of finite presentation, we denote by $X^{\mathrm{sm}}$ the (Zariski-open) relative smooth locus on $X$. We use the same notation in the rigid-analytic category.

A non-archimedean field is a field $k$ equipped with a non-trivial non-archimedean absolute value with respect to which $k$ is complete. An analytic extension field $k^{\prime} / k$ is an extension $k^{\prime}$ of $k$ endowed with a structure of non-archimedean field such that its absolute value extends the one on $k$. All rigid spaces will tacitly be assumed to be over a fixed non-archimedean ground field $k$ unless we say otherwise. We write $\sqrt{ }\left|k^{\times}\right| \subseteq(0, \infty)$ to denote the divisible subgroup of $(0, \infty)$ "generated" by the value group of the absolute value on $k^{\times}$.

When we say GAGA over fields we mean that the analytification functor from proper $k$-schemes to proper rigid-analytic spaces over $k$ is fully faithful, together with the compatibility of analytification with fiber products and the fact that various properties of a map between finite type $k$-schemes (such as being finite, flat, smooth, an isomorphism, or a closed immersion) hold on the algebraic side if and only if they hold on the analytic side. As a special case, if $A$ is a finite $k$-algebra then a proper $A$-scheme (resp. proper rigid space over $\operatorname{Sp}(A)$ ) can be viewed as a proper $k$-scheme (resp. proper rigid space over $k$ ), so when we are restricting attention to the subcategories of objects and morphisms over such an $A$, we shall say GAGA over artin rings. The two other aspects of the GAGA principle that we shall frequently invoke are (i) the elementary fact that $X^{\operatorname{an}}(\operatorname{Sp}(A))=X(\operatorname{Spec} A)$ for any finite $k$-algebra $A$ and any locally finite type $k$-scheme $X$, and (ii) the less trivial fact that 1-dimensional proper rigid-analytic spaces over $k$ are algebraic (this applies to a proper flat rigid-analytic curve over $\operatorname{Sp}(A)$ for a finite $k$-algebra $A)$, the proof of which goes exactly as in the complex-analytic case.

The analytification of a map between locally finite type $k$-schemes is quasiseparated if and only if the scheme map is separated, so to avoid unnecessary separatedness restrictions on the algebraic side when we consider change of the base field on the rigid-analytic side it is convenient to introduce a variant on quasiseparatedness, as follows. A map of rigid spaces $f: X \rightarrow Y$ is pseudo-separated if the diagonal $\Delta_{f}: X \rightarrow X \times_{Y} X$ factors as $X \rightarrow Z \rightarrow X \times_{Y} X$ where the first step is a Zariski-open immersion and the second is a closed immersion. A rigid space $X$ is pseudo-separated if its structure map to $\operatorname{Sp}(k)$ is pseudo-separated. If $X$ and $Y$ are pseudo-separated over $Z$ then any $Z$-map $X \rightarrow Y$ is pseudo-separated, and a map of rigid spaces is pseudo-separated and quasi-separated if and only if it is separated. The reasons for introducing this notion are twofold: (i) analytifications of maps of locally finite type $k$-schemes are always pseudo-separated, and (ii) on the full subcategory of rigid spaces $X$ whose structure morphism to $\operatorname{Sp}(k)$ is a composite of finitely many pseudo-separated maps there is a good "change of 
analytic base field" functor. Pseudo-separatedness is discussed in detail in [C3, $\S A .2]$.

The closed unit ball $\operatorname{Sp}\left(k\left\langle\left\langle t_{1}, \ldots, t_{n}\right\rangle\right\rangle\right)$ over $k$ is denoted $\mathbf{B}_{k}^{n}$, and rigid-analytic affine and projective $n$-spaces over $k$ are denoted $\mathbf{A}_{k}^{n}$ and $\mathbf{P}_{k}^{n}$ respectively; the scheme-theoretic counterparts are denoted $\mathbf{A}_{\operatorname{Spec}(k)}^{n}, \mathbf{P}_{\operatorname{Spec}(k)}^{n}$ on the rare occasions when they arise. For a coherent sheaf $\mathscr{E}$ on a rigid space $S$, we use the Grothendieck convention $\mathbf{P}(\mathscr{E})=\operatorname{Proj}^{\text {an }}(\operatorname{Sym} \mathscr{E})\left(\right.$ with $\operatorname{Proj}^{\text {an }}$ as in $\left.[\mathrm{C} 3,2.3]\right)$, and likewise for quasi-coherent sheaves on schemes.

We freely use the rigid-analytic notions of global irreducible component, geometric irreducibility, geometric reducedness, and geometric connectedness. The reader is referred to $[\mathrm{C} 1]$ for a systematic development of these notions and proofs of more precise results (e.g., compatibility of the notions of irreducibility and connectedness with respect to analytification of locally finite type $k$-schemes). A rigid space $X$ is equidimensional of dimension $d$ if $\operatorname{dim} X_{i}=d$ for each irreducible component $X_{i}$ of $X$, and it is equivalent to say $\operatorname{dim} U=d$ for every non-empty admissible open $U$ in $X$ (or for only $U$ that are irreducible and affinoid); for quasi-separated or pseudo-separated $X$, and an analytic extension field $k^{\prime} / k, X$ is equidimensional with dimension $d$ if and only if $k^{\prime} \widehat{\otimes}_{k} X$ is equidimensional with dimension $d$.

\section{Analytic generalized elliptic CuRves}

The rigid-analytic theory of ampleness [C3] shall be used to develop the theory of generalized elliptic curves over rigid spaces. The basic algebro-geometric theory of generalized elliptic curves is worked out in [DR], and some aspects are briefly reviewed in Appendix B; the reader may wish to skim this appendix before reading this section.

2.1. Basic definitions over a rigid-analytic space. We refer the reader to $\S \mathrm{B} .1$ for a discussion of the algebraic theory of standard Néron polygons over a base scheme. For $n \geq 1$, the standard (Néron) $n$-gon over a rigid space $S$ is $S \times C_{n / k}^{\text {an }}$ where $C_{n / k}$ is the standard Néron $n$-gon over Spec $k$.

Definition 2.1.1. A Deligne-Rapoport $(D R)$ semistable genus-1 curve over a rigid space $S$ is a proper flat morphism $f: C \rightarrow S$ such that for all $s \in S$ the fiber $C_{s}$ is either a smooth and geometrically-connected curve of genus 1 or becomes isomorphic to standard polygon over a finite extension of $k(s)$.

Remark 2.1.2. Since proper 1-dimensional rigid-analytic spaces are algebraic, it follows from GAGA over fields and standard considerations with direct limits of base rings on the algebraic side that if $C$ and $C^{\prime}$ are two proper 1-dimensional rigid-analytic spaces over $k$ that become isomorphic over some analytic extension 
$K / k$ then they become isomorphic over a finite extension of $k$. Hence, using a finite extension of $k(s)$ in Definition 2.1.1 is no different than using an arbitrary algebraically closed analytic extension field of $k(s)$.

Example 2.1.3. Let $\mathscr{C} \rightarrow \mathscr{S}$ be a DR semistable genus-1 curve over a locally finite type $k$-scheme $\mathscr{S}$. This map is projective Zariski-locally over $\mathscr{S}$, so by admissibility of Zariski-open set-theoretic covers of $\mathscr{S}^{\text {an }}$ it follows by elementary arguments that the analytification $\mathscr{C}^{\text {an }} \rightarrow \mathscr{S}^{\text {an }}$ is proper. This map is a DR semistable genus-1 curve over the rigid space $\mathscr{S}^{\text {an }}$.

Definition 2.1.1 is obviously preserved by base change, and it is trivial to check that Theorem B.1.2 carries over to the rigid-analytic case. Somewhat less trivial is the fact that Definition 2.1.1 is preserved under arbitrary change of the base field (which may introduce points that are transcendental with respect to the initial base field). To settle this (see Theorem 2.1.5), we first require the fact that any proper flat rigid-analytic curve over a rigid-analytic space admits a relatively ample line bundle étale-locally on the base:

Theorem 2.1.4. Let $f: C \rightarrow S$ be a proper flat morphism of rigid spaces, and assume that the fibers of $f$ have dimension at most 1 . There exists an admissible covering $\left\{S_{i}\right\}$ of $S$ such that $f_{i}: C_{/ S_{i}} \rightarrow S_{i}$ becomes projective after a quasicompact étale surjective base change on $S_{i}$.

Proof. Without loss of generality $S$ is affinoid, so $C$ and $S$ are quasi-compact and separated. By [BL2, 5.10], there is a formal flat model $\mathfrak{f}: \mathfrak{C} \rightarrow \mathfrak{S}$ of $f$ with $\mathfrak{f}$ having all fibers of dimension at most 1 , and by $[\mathrm{L}, 2.5,2.6] \mathfrak{f}$ is automatically proper since $f=\mathfrak{f}^{\text {rig }}$ is proper. For each $n \geq 0$, let $\mathfrak{f}_{n}: \mathfrak{C}_{n} \rightarrow \mathfrak{S}_{n}$ denote the induced proper, flat, finitely presented morphism over $\operatorname{Spec}\left(R / \mathfrak{I}^{n+1}\right)$, where $R$ is the valuation ring of $k$ and $\mathfrak{I}$ is an ideal of definition of $R$. Note that we may work Zariski-locally on $\mathfrak{S}$, since Zariski-covers of $\mathfrak{S}$ induce admissible open covers of $S$.

Quite generally, if $F: X \rightarrow Y$ is a proper and finitely presented morphism of schemes and all fibers of $F$ have dimension at most 1 , then we claim that there exists a Zariski-open covering $\left\{Y_{i}\right\}$ of $Y$ and quasi-compact étale surjections $Y_{i}^{\prime} \rightarrow Y_{i}$ such that $X \times_{Y} Y_{i}^{\prime}$ admits a $Y_{i}^{\prime}$-ample invertible sheaf for all $i$. In other words, proper curves are projective étale-locally on the base. By standard limit arguments, it suffices to prove that if $Y$ is henselian local then $F$ is projective. Let us grant this for a moment and use it to complete the proof.

Taking $F: X \rightarrow Y$ to be $\mathfrak{f}_{0}: \mathfrak{C}_{0} \rightarrow \mathfrak{S}_{0}$, by passing to small opens in $\mathfrak{S}_{0}$ (or $\mathfrak{S})$ we may suppose that there exists a quasi-compact étale surjection $\mathfrak{S}_{0}^{\prime} \rightarrow \mathfrak{S}_{0}$ and an $\mathfrak{f}_{0}^{\prime}$-ample invertible sheaf $\mathscr{L}_{0}$ on $\mathfrak{C}_{0}^{\prime}$, with $\mathfrak{S}_{0}$ affine. Let $\mathfrak{S}_{n}^{\prime} \rightarrow \mathfrak{S}_{n}$ be the unique étale lifting of $\mathfrak{S}_{0}^{\prime} \rightarrow \mathfrak{S}_{0}$, so the $\mathfrak{S}_{n}^{\prime}$ 's define a flat formal scheme morphism $\mathfrak{S}^{\prime} \rightarrow \mathfrak{S}$ with $\mathfrak{S}^{\prime}$ topologically finitely presented over $R$. Let $S^{\prime} \rightarrow S$ be 
the corresponding flat morphism of quasi-compact rigid spaces. The surjectivity and flatness of the formal scheme morphism $\mathfrak{S}^{\prime} \rightarrow \mathfrak{S}$ forces the flat rigid-analytic morphism $S^{\prime} \rightarrow S$ to be surjective (by Lemma A.1.5).

The coherent $\mathscr{O}_{\mathfrak{S}^{\prime} \text {-module }} \widehat{\Omega}_{\mathfrak{S}^{\prime} / \mathfrak{S}}^{1}$ vanishes and induces $\Omega_{S^{\prime} / S}^{1}$ on $S^{\prime}$, so the quasi-compact flat surjection $S^{\prime} \rightarrow S$ is étale. Replacing $\mathfrak{S}$ with $\mathfrak{S}^{\prime}$, we may assume that an $\mathfrak{f}_{0}$-ample $\mathscr{L}_{0}$ exists on $\mathfrak{C}_{0}$. Since $\mathfrak{f}_{0}$ is proper, flat, and finitely presented, by cohomology and base change we can replace $\mathscr{L}_{0}$ with a large tensor power so that $\mathscr{L}_{0}$ has vanishing degree- 1 cohomology on fibers and $\mathscr{L}_{0}$ is very ample. Working locally on $\mathfrak{S}$, we can assume that $\mathfrak{S}$ is affine. Since the $\mathfrak{f}_{n}$ 's are proper of finite presentation with fibers of dimension at most 1 , and the $\mathfrak{S}_{n}$ 's are affine, $\mathrm{H}^{2}\left(\mathfrak{C}_{n}, \mathscr{F}\right)=0$ for any quasi-coherent sheaf $\mathscr{F}$ on $\mathfrak{C}_{n}$ for any $n \geq 0$ (use direct limits and the theorem on formal functions). Thus, there exist compatible invertible sheaves $\mathscr{L}_{n}$ on all $\mathfrak{C}_{n}$ 's. Since each $\mathfrak{f}_{n}$ is proper, flat, and finitely presented, by cohomology and base change (and the cohomological vanishing hypothesis for $\mathscr{L}_{0}$ ) it follows that the $\mathscr{L}_{n}$ 's define a closed immersion of the proper $\mathfrak{C}$ into a formal projective space over $\mathfrak{S}$. Now apply $(\cdot)^{\text {rig }}$ to get a closed immersion of $C$ into a projective space over $S$.

It remains to prove the above general assertion concerning projectivity of proper finitely presented morphisms $F: X \rightarrow \operatorname{Spec} A$ with fibers of dimension at most 1 over a henselian local base Spec $A$. Using standard direct limit arguments [EGA, $\mathrm{IV}_{3}, \S 8 f f$.], we reduce to the case when $A$ is also noetherian. We need to construct an ample invertible sheaf $\mathscr{L}$ on $X$. By [EGA, $\left.\mathrm{IV}_{3}, 9.6 .4\right]$, it is necessary and sufficient to construct an invertible sheaf on $X$ that is ample on the closed fiber.

There is a quick construction of such an $\mathscr{L}$ using the Artin approximation theorem and the Grothendieck Existence Theorem, but here is a more elementary construction suggested by J. Starr. We can assume that $X$ is connected, so by the henselian property of $A$ it follows that the closed fiber $X_{0}$ is connected. If the closed fiber is 0 -dimensional then the open quasi-finite locus [EGA, $\mathrm{IV}_{3}, 13.1 .4$ ] must fill up all of the proper $X$ and hence $X$ is $A$-finite, so $\mathscr{O}_{X}$ is relatively ample. Thus, we may suppose that $X_{0}$ has pure dimension 1 . For each irreducible component of the fiber $X_{0}$ over the reduced closed point, choose a closed point $x_{i}$ on this component such that $x_{i}$ is not on any other irreducible component of $X_{0}$. Also make sure that each $x_{i}$ is not one of the finitely many associated points of $X$ or of $X_{0}$. Finally, we may suppose that if $x_{i}$ lies in the closure of an associated point $\eta_{j}$ of $X$ then the closure of $\eta_{j}$ contains the entire irreducible component of $X_{0}$ on which $x_{i}$ lives. In the local ring $B_{i}=\mathscr{O}_{X, x_{i}}$ at $x_{i}$, let $I_{i}$ denote the stalk of the ideal sheaf of $X_{0}$, so the associated primes $\mathfrak{p}_{j}$ of $B_{i}$ all contain $I_{i}$ but lie strictly inside of the maximal ideal $\mathfrak{m}_{i}$. Thus, the prime ideals $\mathfrak{p}_{j} / I_{i}$ in $B_{i} / I_{i}$ and the associated primes of $B_{i} / I_{i}$ are all strictly contained inside of the maximal ideal. By prime avoidance, we may select a non-unit $g_{i}$ in $B_{i}$ whose image in 
$B_{i} / I_{i}$ lies outside of all of these primes. Thus, on both $X$ and $X_{0}$ the germ $g_{i}$ is not a zero divisor at $x_{i}$.

Smearing out $g_{i}$ over a neighborhood of $x_{i}$, its zero-scheme defines a subscheme $Z_{i}$ of $X$ passing through $x_{i}$ that is quasi-finite (over the henselian local base) at $x_{i}$. Since $Z_{i}$ is separated over a henselian local ring, it follows from Zariski's Main Theorem that the connected component $Z_{i}^{\prime}$ of $x_{i}$ in $Z_{i}$ is finite over the base. The scheme $Z_{i}^{\prime}$ is then clearly a local closed subscheme of $X$ whose ideal sheaf is invertible, so the ideal sheaf on $X$ of the disjoint union $D$ of the $Z_{i}^{\prime}$ 's is invertible and (by the way we selected the $g_{i}$ 's) restricts to the invertible ideal sheaf of $D_{0}=D \cap X_{0}$ in $X_{0}$. Thus, the inverse $\mathscr{L}$ of the ideal sheaf of $D$ is ample.

Theorem 2.1.5. Let $f: C \rightarrow S$ be a DR semistable genus-1 curve over a quasiseparated or pseudo-separated rigid space $S$. Let $k^{\prime} / k$ be an analytic extension field, and let $f^{\prime}: C^{\prime} \rightarrow S^{\prime}$ be the relative rigid-analytic curve obtained by extension of the base field from $k$ to $k^{\prime}$. The map $C^{\prime} \rightarrow S^{\prime}$ is a DR semistable genus-1 curve over the rigid space $S^{\prime}$ over $k^{\prime}$.

See $\S 1.3$ for the definition of pseudo-separatedness.

Proof. The problem is local on $S$, and clearly a proper rigid space over $S^{\prime}$ is a DR semistable genus-1 curve over $S^{\prime}$ if and only if it is one after a quasi-compact étale surjective base change on $S^{\prime}$. Moreover, by Theorem A.2.4(d),(k),(n), for any quasi-compact étale surjection $T \rightarrow S$ the map $T^{\prime} \rightarrow S^{\prime}$ of rigid spaces over $k^{\prime}$ is also quasi-compact, étale, and surjective. Thus, by Theorem 2.1.4 we may assume that there exists a closed $S$-immersion $C \hookrightarrow \mathbf{P}_{k}^{n} \times S$. By the rigid-analytic representability properties of the Hilbert functor [C3, Thm. 4.1.3] (especially compatibility with analytification), there exists a proper flat curve $\mathscr{C} \rightarrow \mathscr{S}$ over a locally finite type $k$-scheme $\mathscr{S}$ and a map $\alpha: S \rightarrow \mathscr{S}$ an that pulls $\mathscr{C}^{\text {an }}$ back to $C$. The composite map $S \rightarrow \mathscr{S}$ must land inside of the locus over which the fibers of $\mathscr{C} \rightarrow \mathscr{S}$ are DR semistable genus-1 curves. By Lemma B.1.3, this locus is an open subscheme $\mathscr{U} \subseteq \mathscr{S}$.

Let $\mathscr{Z}$ be a closed subscheme structure on the complement of $\mathscr{U}$ in $\mathscr{S}$, and let $\mathscr{S}^{\prime}=k^{\prime} \otimes_{k} \mathscr{S}$. The rigid space $\mathscr{S}^{\text {an }}$ is pseudo-separated and there is a natural isomorphism $k^{\prime} \widehat{\otimes}_{k}\left(\mathscr{S}^{\text {an }}\right) \simeq \mathscr{S}^{\text {an }}$. Under the map

$$
\alpha^{\prime}: S^{\prime} \rightarrow k^{\prime} \widehat{\otimes}_{k}\left(\mathscr{S}^{\mathrm{an}}\right) \simeq \mathscr{S}^{\text {an }}
$$

the pullback of $k^{\prime} \widehat{\otimes}_{k}\left(\mathscr{Z}^{\text {an }}\right) \simeq \mathscr{Z}^{\text {an }}$ is empty. Zariski-openness in rigid geometry is compatible with change of the base field [C1, 3.1.1], so the map $\alpha^{\prime}$ factors through the Zariski-open locus $k^{\prime} \widehat{\otimes}_{k}\left(\mathscr{U}^{\text {an }}\right) \simeq \mathscr{U}^{\text {an }}$. Since $\mathscr{C}^{\prime} \rightarrow \mathscr{U}^{\prime}$ is a DM semistable genus-1 curve, the pullback of $\mathscr{C}^{\prime \text { an }} \rightarrow \mathscr{U}^{\text {an }}$ along $\alpha^{\prime}: S^{\prime} \rightarrow \mathscr{U}^{\prime \text { an }}$ is a DR semistable genus-1 curve over $S^{\prime}$. This pullback is exactly $C^{\prime} \rightarrow S^{\prime}$. 
Using properties of the rigid-analytic Proj and Hilbert functors as in [C3, Thm. 2.3.8, Thm. 4.1.3], particularly the good behavior of rigid-analytic Proj with respect to flatness and change of the ground field, one can carry over (essentially verbatim) the scheme-theoretic methods used to construct contractions as in (B.1.1); see Remark B.1.4. More precisely:

Theorem 2.1.6. Let $f: C \rightarrow S$ be a DR semistable genus-1 curve over a rigid space $S$ and let $D \hookrightarrow C$ be a relative effective Cartier divisor that is finite flat over $S$ and lies in $C^{\mathrm{sm}}$. Assume that $D \rightarrow S$ is surjective.

There exists a unique map $u: C \rightarrow \bar{C}$, where $\bar{C}_{/ S}$ is a DR semistable genus-1 curve and on geometric fibers over $S$ the map $u$ is the contraction of precisely the irreducible components not meeting the fiber of D. The map $u$ is unique up to unique isomorphism and it is functorial with respect to isomorphisms in the pair $(C, D)$.

The contraction map $u: C \rightarrow \bar{C}$ is compatible with base change on $S$, with analytification from the scheme case, and with change of the base field when $S$ is quasi-separated or pseudo-separated.

Remark 2.1.7. By geometric-fiber considerations, $u^{-1}\left(\bar{C}^{\mathrm{sm}}\right)$ is contained in $C^{\mathrm{sm}}$ and the restriction

$$
u^{\prime}: u^{-1}\left(\bar{C}^{\mathrm{sm}}\right) \rightarrow \bar{C}^{\mathrm{sm}}
$$

of $u$ is quasi-compact (even proper) and an isomorphism on fibers over $S$, so $u^{\prime}$ is an isomorphism by Corollary A.2.6(1). That is, $u$ identifies $\bar{C}^{\mathrm{sm}}$ with an admissible open in $C^{\mathrm{sm}}$.

Definition 2.1.8. A generalized elliptic curve over a rigid space $S$ is a triple $(E,+, e)$ where $f: E \rightarrow S$ is a DR semistable genus-1 curve over $S$ and the data $+: E^{\mathrm{sm}} \times_{S} E \rightarrow E$ and $e \in E^{\mathrm{sm}}(S)$ satisfy the two properties:

- + restricts to a commutative $S$-group structure on $E^{\mathrm{sm}}$ with identity section $e$,

- + is an action of $E^{\mathrm{sm}}$ on $E$ such that for each non-smooth fiber $E_{s}$ and algebraically closed extension $\bar{s}: k(s) \rightarrow K$, the translation action by any element of $E_{\bar{s}}^{\mathrm{sm}}(\bar{s})$ induces a rotation on the graph of irreducible components of $E_{\bar{s}}$.

Remark 2.1.9. By GAGA over fields and the theory of geometric irreducibility and geometric connectivity in [C1], each $E_{s}^{\mathrm{sm}}$ has only finitely many irreducible components and hence there exists a finite extension $k^{\prime} / k(s)$ such that the irreducible components over $k^{\prime}$ are geometrically irreducible (and correspond to the irreducible components of $\left.E_{s / k^{\prime}}\right)$. In the second axiom for + in Definition 2.1.8, it suffices to replace the arbitrary $K$ with a finite extension $k^{\prime} / k(s)$ such that each irreducible component of $E_{s / k^{\prime}}^{\mathrm{sm}}$ has a $k^{\prime}$-rational point. 
Example 2.1.10. Let $(\mathscr{E} \rightarrow \mathscr{S},+, e)$ be a generalized elliptic curve over a locally finite type $k$-scheme. By Example 2.1.3, $\mathscr{E}^{\text {an }} \rightarrow \mathscr{S}^{\text {an }}$ is a DR semistable genus-1 curve over the rigid space $\mathscr{S}^{\text {an }}$. Equipped with $+^{\text {an }}$ and $e^{\text {an }}$, it is a generalized elliptic curve over $\mathscr{S}^{\text {an }}$.

The relative smooth locus $E^{\mathrm{sm}}$ in Definition 2.1.8 is the Zariski-open locus where the coherent $\Omega_{E / S}^{1}$ on $E$ is locally free of rank 1 . The formation of the locus where a coherent sheaf on a rigid space is locally free with a specified rank is compatible with change of the base field [C3, Cor. A.2.7], so the formation of $E^{\mathrm{sm}}$ is compatible with change of the base field. In view of Theorem 2.1.5, the only obstruction to deducing immediately that (for quasi-separated or pseudoseparated $S$ ) arbitrary analytic extension of the base field carries generalized elliptic curves to generalized elliptic curves is the issue of the rotation condition on geometric fibers. This seems to be a rather hard problem to treat at the outset, except when the base $S$ is 0 -dimensional, and we will settle it affirmatively after we have done some work on the global structure of rigid-analytic families of generalized elliptic curves. Thus, results concerning behavior with respect to arbitrary change in the ground field are postponed until the end of $\S 2.2$.

When $S=\operatorname{Sp}(k)$, the identity component of $E^{\mathrm{sm}}$ has a rational point and hence it is geometrically connected [C1, 3.2.1]. Thus, for any $S$ and $s \in S$ the notion of "identity component" of a fiber $E_{s}^{\mathrm{sm}}$ is well-behaved with respect to base change on $S$ and arbitrary analytic extension on $k(s)$ for $s \in S$. We begin our study of rigid-analytic generalized elliptic curves with an analogue of [DR, II, 2.7].

Theorem 2.1.11. Let $f: E \rightarrow S$ be a DR semistable genus-1 curve over a rigid space $S$, and assume that $f$ has geometrically irreducible fibers. Let $e \in E^{\mathrm{sm}}(S)$ be a section. There is a unique structure of generalized elliptic curve on $E \rightarrow S$ with identity section e.

Proof. The uniqueness assertion and fpqc-descent for morphisms [C3, Thm. 4.2.3] permit us to work fpqc-locally on $S$ for existence. Thus, by using the argument with Hilbert functors in the proof of Theorem 2.1.5, we may suppose that the marked curve $(E, e)$ is the pullback of a DR semistable marked genus-1 curve $\mathscr{E} \rightarrow \mathscr{S}$ over a locally finite type $k$-scheme $\mathscr{S}$ (the marking being a choice of section in $\mathscr{E} \mathrm{sm}(\mathscr{S})$ ). The locus $\mathscr{U} \subseteq \mathscr{S}$ of geometrically integral fibers for $\mathscr{E}$ is Zariski-open [EGA, $\mathrm{IV}_{3}, 12.2 .4$ ] and the map $S \rightarrow \mathscr{S}^{\text {an }}$ must land inside of $\mathscr{U}^{\text {an }}$. By the algebraic analogue [DR, II, 2.7] of the present theorem, $\left.\mathscr{E}\right|_{\mathscr{U}} \rightarrow \mathscr{U}$ with its marked section admits a unique structure of generalized elliptic curve, so we get existence fpqc-locally on the original $S$.

The remaining issue is to prove uniqueness. We use Corollary A.2.6(3) to reduce to the case of an artin local base $S=\operatorname{Sp}\left(A_{0}\right)$, so $E$ is a proper 1-dimensional 
rigid space over $k$. Let $E^{\text {alg }}$ be the unique corresponding proper $A_{0}$-scheme under GAGA over artin rings, so $E^{\text {alg }}$ is naturally a DR semistable genus- 1 curve over $\operatorname{Spec}\left(A_{0}\right)$. Clearly $E^{\text {alg }} \rightarrow \operatorname{Spec}\left(A_{0}\right)$ has geometrically integral fibers. Let $e^{\text {alg }} \in\left(E^{\mathrm{alg}}\right)\left(\operatorname{Spec}\left(A_{0}\right)\right)=E\left(\operatorname{Sp}\left(A_{0}\right)\right)$ be the unique section whose analytification is $e$ under the GAGA correspondence over artin rings. We are trying to compare two maps

$$
+^{\prime},+^{\text {an }}: E^{\mathrm{sm}} \times_{S} E \rightrightarrows E,
$$

where $+^{\prime}$ is an abstract rigid-analytic generalized elliptic curve structure with identity section $e$ and $+{ }^{\text {an }}$ is the analytification of the unique such structure + on $\left(E^{\text {alg }}, e^{\text {alg }}\right)$. When $E^{\mathrm{sm}}=E$ is proper we may use GAGA over artin rings to reduce to the known algebraic case. Thus, we now suppose that the irreducible geometric fiber over the artin local base is a 1-gon.

Our comparison between $+^{\prime}$ and $+^{\text {an }}$ rests on the following variant of Corollary A.2.6(3) that is an immediate consequence of Krull's intersection theorem:

Lemma 2.1.12. Let $X, Y$ be rigid spaces over $S$. For two $S$-maps $f, g: X \rightrightarrows Y$, $f=g$ if and only if the induced maps $X\left(S_{0}\right) \rightrightarrows Y\left(S_{0}\right)$ coincide for all local artin objects $S_{0} \rightarrow S$ over $S$.

Rather than directly prove equality of the two maps in (2.1.1), let us first check the $a$ priori weaker claim that the two group laws $+^{\prime},{ }{ }^{\mathrm{an}}: E^{\mathrm{sm}} \times E^{\mathrm{sm}} \rightrightarrows E^{\mathrm{sm}}$ on the smooth locus are the same. To compare the group laws $+^{\prime}$ and $+^{\text {an }}$ on $E^{\mathrm{sm}}$ over $S=\mathrm{Sp}\left(A_{0}\right)$, by Lemma 2.1.12 it suffices to show (by allowing the artin local $A_{0}$ to vary) that for $x \in E^{\mathrm{sm}}(S)$ the translation action $x+{ }^{\prime}$ on $E^{\mathrm{sm}}$ over $S$ is the same as $x+{ }^{\text {an }}$. But $E^{\mathrm{sm}}\left(A_{0}\right)=\left(E^{\mathrm{alg}}\right)^{\mathrm{sm}}\left(A_{0}\right)$, so $x=y^{\text {an }}$ for a unique $A_{0}$-section $y$ of $\left(E^{\mathrm{alg}}\right)^{\mathrm{sm}}$. It follows that $x+^{\text {an }}=(y+)^{\text {an }}$ on $E$. Thus, we need to prove that the automorphism $x+{ }^{\prime}$ of $E=\left(E^{\mathrm{alg}}\right)^{\text {an }}$ algebraizes (under GAGA over artin rings) to the translation action $y+$ on $E^{\text {alg }}$. If $x+^{\prime}$ merely algebraizes to some translation $z+$ with $z \in\left(E^{\mathrm{alg}}\right)^{\mathrm{sm}}\left(A_{0}\right)$ then acting on the algebraization $e^{\text {alg }}$ of $e$ forces $z^{\text {an }}=x+^{\prime} e=x=y^{\text {an }}$, so $z=y$. Thus, to compare $+^{\prime}$ and $+^{\text {an }}$ as groups laws on $E^{\mathrm{sm}}$ with identity $e$, we just need to prove that the algebraization of $x+{ }^{\prime}$ to an automorphism of $E^{\text {alg }}$ is a +-translation.

Since the geometric fiber of $E^{\text {alg }}$ is a 1-gon, Theorem B.2.5 applied to $E^{\text {alg }} \rightarrow$ Spec $A_{0}$ with $G=\left\{e^{\text {alg }}\right\}$ ensures that every automorphism of $E^{\text {alg }}$ over Spec $A_{0}$ is a + -translation. This completes the proof of the weaker claim that $+^{\prime}=+^{\text {an }}$ as group laws on $E^{\mathrm{sm}}$ over $\operatorname{Sp}\left(A_{0}\right)$.

We conclude that for $x \in E^{\mathrm{sm}}(S)$ the translation maps $x+^{\prime}, x+{ }^{\text {an }}: E \rightrightarrows E$ over $S$ coincide on $E^{\mathrm{sm}}$, and so they coincide on $E$ because it suffices to compare the algebraizations $E^{\text {alg }} \rightrightarrows E^{\text {alg }}$ of these two analytic automorphisms $E \rightrightarrows E$ via GAGA over artin rings (equality holds on the algebraic side because $\left(E^{\mathrm{alg}}\right)^{\mathrm{sm}}$ is 
relatively schematically dense in $\left.E^{\text {alg }}\left[\mathrm{EGA}, \mathrm{IV}_{3}, 11.10 .8 f f.\right]\right)$. Thus, $x+^{\prime}=x+^{\text {an }}$ for any $x \in E^{\mathrm{sm}}(S)$ when $S=\operatorname{Sp}\left(A_{0}\right)$ is artin local.

The same arguments as in the scheme case show that for any generalized elliptic curve $E_{/ S}$ and subgroup object $D \hookrightarrow E^{\mathrm{sm}}$ that is finite flat over $S$, the contraction $\bar{E}$ of $E$ away from $D$ (in the sense of Theorem 2.1.6) admits a unique compatible structure of generalized elliptic curve. In particular, taking $D$ to be the identity section, we see that the contraction $c(E)$ away from the identity section has a unique generalized elliptic curve structure compatible with that on $E$. But $c(E)$ has geometrically irreducible fibers, so its generalized elliptic curve structure is uniquely determined by its identity section (without reference to $E$ ), by Theorem 2.1.11. This allows us to prove:

Corollary 2.1.13. If $k$ is algebraically closed then up to isomorphism the only rigid-analytic generalized elliptic curves over $\operatorname{Sp}(k)$ are smooth elliptic curves and the analytifications of the standard polygons with their standard algebraic generalized elliptic curve structure.

In general, if $E$ is a non-smooth rigid-analytic generalized elliptic curve over a non-archimedean field $k$ then it becomes isomorphic to a standard n-gon endowed with its standard generalized elliptic curve structure over a finite extension of $k$ (that may be chosen to be separable over $k$ if $\operatorname{char}(k) \nmid n)$.

Proof. The smooth case is trivial, by GAGA over fields, so we may restrict attention to the non-smooth case. Also, if $E$ is a rigid-analytic generalized elliptic curve over an arbitrary non-archimedean field $k$ and $E$ is non-smooth, then under the GAGA correspondence over fields the algebraization of $E$ is a DR semistable genus-1 curve over the field $k$. By the algebraic theory of such curves, in such cases we can find a finite separable extension of $k$ over which the curve becomes isomorphic to a standard polygon $C_{n}$. Thus, it suffices to consider the case of an arbitrary $k$ and a generalized elliptic curve structure $+^{\prime}$ on a standard polygon over $\operatorname{Sp}(k)$ with the identity point $e$ lying on the component labelled by $0 \in \mathbf{Z} / n \mathbf{Z}$. Using a $k^{\times}$-multiplier on the entire polygon, we can assume $e=1$ on this component of the standard polygon. We want to prove that, at least over a finite extension of $k$ (separable if $\operatorname{char}(k) \nmid n$ ) there is an analytic (and so necessarily algebraic) automorphism of the rigid-analytic marked curve $\left(C_{n}, e\right)$ carrying the structure $+^{\prime}$ to the standard generalized elliptic curve structure + .

By Theorem 2.1.11, the case of 1-gons is settled. Thus, consider the standard $n$-gon $C_{n}$ with $n \geq 2$. Let $E$ denote $C_{n}$ endowed with the structure $+^{\prime}$. The contraction $c(E)$ away from the identity component is a standard 1-gon with generalized elliptic curve structure having 1 as the identity, so by Theorem 2.1.11 we know $c(E)^{\mathrm{sm}}=\mathbf{G}_{m}^{\text {an }}$ as an analytic group (compatibly with the canonical identification of the marked curve $(c(E), c(e))$ with the standard 1-gon with marking 
at the point 1). The contraction map identifies $c(E)^{\mathrm{sm}}$, as an analytic group, with the identity component of the group object $E^{\mathrm{sm}}$ (see Remark 2.1.7). Hence, the group structure on the identity component is uniquely determined.

The "rotation" hypothesis in the definition of generalized elliptic curve implies that the finite constant component group is cyclic with a generating component given by either component adjacent to the identity component. Let $C$ be the irreducible component labelled by $1 \in \mathbf{Z} / n \mathbf{Z}$, so abstractly $C$ is a rigid-analytic projective line over $k$. Pick a $k$-rational point $x \in C$ avoiding the two points in $C$ that are not in $E^{\mathrm{sm}}$. By replacing $k$ with a finite extension generated by an $n$th root of $[n] x \in \mathbf{G}_{m}^{\text {an }}(k)=k^{\times}$, we can assume that $[n] x \in \mathbf{G}_{m}^{\text {an }}(k)$ is equal to $[n] y$ for some $y \in \mathbf{G}_{m}^{\mathrm{an}}(k)=c(E)^{\mathrm{sm}}(k)$. Under the canonical identification of $c(E)^{\mathrm{sm}}$ with the identity component of $E^{\mathrm{sm}}$ we may view $y$ as a rational point on this identity component. Replacing $x$ with $x y^{-1}$ brings us to the case $[n] x=e$, so we get a unique isomorphism of analytic groups $\varphi: E^{\mathrm{sm}} \simeq C_{n}^{\mathrm{sm}}=\mathbf{G}_{m}^{\mathrm{an}} \times \mathbf{Z} / n \mathbf{Z}$ via $c(E)^{\mathrm{Sm}}=\mathbf{G}_{m}^{\text {an }}$ and $x \mapsto(1,1)$. This isomorphism is algebraic by construction (since the abstract analytic automorphism $x+{ }^{\prime}$ of $E$ is algebraic by GAGA over fields), so it uniquely extends to an analytic isomorphism $E \simeq C_{n}$ that, for density reasons, carries $+^{\prime}$ to + .

Corollary 2.1.14. Let $E$ be a rigid-analytic generalized elliptic curve over $k$ and let $E^{\text {alg }}$ be the associated DR semistable genus-1 curve over $\operatorname{Spec}(k)$. The generalized elliptic curve structure on $E$ uniquely algebraizes to a generalized elliptic curve structure on $E^{\mathrm{alg}}$.

Proof. The uniqueness follows from the faithfulness of the analytification functor. Since the smooth locus on $E^{\text {alg }}$ analytifies to the smooth locus on $E$, the only problem is to prove that the morphism $+: E^{\mathrm{sm}} \times E \rightarrow E$ is algebraic (as then $e \in$ $E(k)=E^{\text {alg }}(k)$ provides the identity point on the algebraic side, and preservation of irreducible component decomposition under analytification takes care of the rotation requirement in the non-smooth case). The algebraicity in the smooth case follows from GAGA over fields, so we may assume $E$ is not smooth. By fpqc descent theory for morphisms on the rigid-analytic and scheme-theoretic sides, it suffices to check the algebraicity after a finite extension of the base field. Thus, the final part of Corollary 2.1.13 solves the problem (since a rigid-analytic isomorphism from $E$ to a standard polygon is necessarily algebraic, by GAGA over fields).

2.2. Global structure of generalized elliptic curves over rigid spaces. The starting point for the global theory is the fact that Theorem B.2.6 carries over to the rigid-analytic case:

Theorem 2.2.1. Let $C \rightarrow S$ be a DR semistable genus-1 curve over a rigid space $S$ and let $D \hookrightarrow C$ be an $S$-ample relative effective Cartier divisor supported in 
$C^{\mathrm{sm}}$. Assume that the finite flat $D_{/ S}$ is endowed with a structure of commutative group object and that there is given an action $\rho: D \times C \rightarrow C$ that restricts to the group law on $D$.

This data extends to a generalized elliptic curve structure on $C$ if and only if it does so on fibers, in which case such a structure is unique.

Since the fibers of $C \rightarrow S$ have unique projective algebraic structure, by Corollary 2.1.14 and GAGA (over fields) applied to $\rho$ on the fibers it follows that the $\mathrm{Pic}^{0}$-criterion in Theorem B.2.6 gives a necessary and sufficient condition for the verification of the criterion in Theorem 2.2.1 that there exists a generalized elliptic curve structure (compatible with $\rho$ ) on the fibers. Likewise, it suffices to have the existence of such a structure on each geometric fiber.

Proof. We first prove uniqueness, and for this purpose it is sufficient to work on infinitesimal fibers ( $c f$. proof of Lemma 2.1.12). Thus, we may and do now assume $S=\operatorname{Sp}\left(A_{0}\right)$ with a finite local $k$-algebra $A_{0}$, so the rigid space $C$ has dimension 1. The idea is to imitate the proof of uniqueness in Theorem 2.1.11, so we have to first establish an algebraic existence result over $\operatorname{Spec}\left(A_{0}\right)$. Since proper rigid-analytic spaces with dimension $\leq 1$ are algebraic, we let $D^{\text {alg }}$ and $C^{\text {alg }}$ be the associated algebraizations of $D$ and $C$. By GAGA over artin rings, $C^{\text {alg }}$ is a DR semistable genus- 1 curve over $\operatorname{Spec}\left(A_{0}\right)$ and $D^{\text {alg }}$ is a $\operatorname{Spec}\left(A_{0}\right)$-ample relative effective Cartier divisor supported in its smooth locus such that the $S$ group structure on $D$ uniquely algebraizes to a commutative $A_{0}$-group scheme structure on $D^{\text {alg }}$ and $\rho$ uniquely algebraizes to an action $\rho^{\text {alg }}$ of $D^{\text {alg }}$ on $C^{\text {alg }}$ over $\operatorname{Spec}\left(A_{0}\right)$ extending the group scheme structure on $D^{\text {alg }}$. By the existence and uniqueness results in the algebraic case (Theorem B.2.6), $\rho^{\text {alg }}$ has at most one extension to a structure of generalized elliptic curve on $C^{\text {alg }}$ over $\operatorname{Spec}\left(A_{0}\right)$ and it admits one if and only if $\rho^{\text {alg }} \otimes_{A_{0}} k_{0}$ does so for $C^{\text {alg }} \otimes_{A_{0}} k_{0}$ over $\operatorname{Spec}\left(k_{0}\right)$. Over $k_{0}$ the algebraic existence follows from Corollary 2.1.14 and the assumption that $\rho_{0}=\rho \otimes A_{0} k_{0}$ extends to a generalized elliptic curve structure on $C_{0}=C \otimes A_{0} k_{0}$.

Note that there can be at most one generalized elliptic curve structure on $C_{0}$ compatible with $\rho_{0}$ because Corollary 2.1.14 ensures that any such structure is automatically algebraic and the resulting algebraization is necessarily compatible with $\rho_{0}^{\text {alg }}$ (so the uniqueness aspect in Theorem B.2.6 gives uniqueness for this algebraized structure). We conclude that the generalized elliptic curve structure that is assumed to exist on $C_{0}$ compatibly with $\rho_{0}$ is unique and its algebraization to a generalized elliptic curve structure on $C_{0}^{\text {alg }}$ over $\operatorname{Spec}\left(k_{0}\right)$ uniquely deforms to a $\rho^{\text {alg }}$-compatible generalized elliptic curve structure + on $C^{\text {alg }}$ over $\operatorname{Spec}\left(A_{0}\right)$. In particular, $+{ }^{\text {an }}$ gives a solution to the existence problem over $\operatorname{Sp}\left(A_{0}\right)$, so the original uniqueness problem is equivalent to the assertion that any solution $+^{\prime}$ to the existence problem must be equal to the analytified structure $+{ }^{\text {an }}$ over $\operatorname{Sp}\left(A_{0}\right)$. Compatibility with $\rho$ forces any such $+^{\prime}$ to have identity section given by that of 
$D$ (and so this identity section algebraizes to the identity section of the unique solution + to the algebraic existence problem on $C^{\text {alg }}$ ).

Since we have agreement of identity sections for $+^{\prime}$ and $+{ }^{\text {an }}$, we are now in exactly the same situation as (2.1.1), except that the fiber $C_{0}$ may not be geometrically irreducible. The only purpose of the geometric irreducibility condition in the analysis of (2.1.1) is to use $G=\left\{e^{\text {alg }}\right\}$ in the confirmation that for any $x \in C^{\operatorname{sm}}(S)$ the algebraization of the automorphism $x+{ }^{\prime}$ of $C$ is a +-translation on $\left(C^{\text {alg }},+\right)$. By the uniqueness in Theorem B.2.6, we may apply Theorem B.2.5 with $G=D^{\text {alg }}$ and $\rho=\rho^{\text {alg }}$ to complete the general uniqueness proof in the rigid-analytic case.

The problem is now one of global existence when existence is given on fibers, so we may work locally on $S$. Thus, we may assume the finite flat covering $D \rightarrow S$ has constant rank $d>0$. The rigid-analytic version of Theorem B.1.2 and the compatibility of representing objects for the analytic and algebraic Hilbert functors for projective space [C3, Thm. 4.1.3] allow us to suppose (by further localization on $S$ ) that $C$ is the pullback of a universal curve $\mathscr{C}$ over a suitable Hilbert scheme $\mathscr{H}$ over $\operatorname{Spec}(k)$. There is a scheme $\mathscr{H}^{(d)}$ of finite type over $\mathscr{H}$ such that $\mathscr{H}^{(d)}$ is universal for the specification of a degree- $d$ relative effective Cartier divisor $\mathscr{D}$ in $\mathscr{C}$ equipped with a structure of commutative group object. By $\left[\right.$ EGA, $\left.\mathrm{IV}_{3}, 9.6 .4\right]$, there is an open subscheme $\mathscr{U} \subseteq \mathscr{H}^{(d)}$ that is universal for the conditions that $\mathscr{D}$ is relatively ample and supported in $\mathscr{C}^{\mathrm{sm}}$.

Over $\mathscr{U}$ we may consider the Hom-scheme $\operatorname{Hom}(\mathscr{D} \times \mathscr{C}, \mathscr{C})$ and form several fiber products with it so as to universally make an action $\alpha: \mathscr{D} \times \mathscr{C} \rightarrow \mathscr{C}$ of the group scheme $\mathscr{D}$ on the curve $\mathscr{C}$ extending the group law on $\mathscr{D}$ over a universal base $\mathscr{S}$ that is locally of finite type over $k$. The analytification compatibilities for Spec, Hilbert, and Hom functors [C3, Thm. 2.2.5(3), Thm. 4.1.3, Cor. 4.1.5] ensure that the analytification of the universal structure $\alpha$ over $\mathscr{S}$ in the category of $k$-schemes is likewise universal in the rigid-analytic category over $k$. Put another way, the data consisting of

- the DR semistable genus 1 curve $C_{/ S}$,

- the relatively ample degree- $d$ relative effective Cartier divisor $D \hookrightarrow C$ supported in the smooth locus and endowed with a commutative group structure,

- the action map $\rho: D \times C \rightarrow C$ extending the group structure

is the pullback to $S$ of the set of data $\left(\mathscr{C}^{\text {an }}, \mathscr{D}^{\text {an }}, \alpha^{\text {an }}\right)$ over $\mathscr{S}^{\text {an }}$. We can apply Lemma B.1.3 and Theorem B.2.6 to the universal structure $(\mathscr{C}, \mathscr{D}, \alpha)$ over $\mathscr{S}$ in order to find a Zariski-open $\mathscr{V} \subseteq \mathscr{S}$ that is the universal $\mathscr{S}$-scheme over which $\alpha: \mathscr{D} \times \mathscr{C} \rightarrow \mathscr{C}$ extends to a generalized elliptic curve structure $\mathscr{C}^{\mathrm{sm}} \times \mathscr{S} \mathscr{C} \rightarrow$ $\mathscr{C}$. The initial hypothesis of fibral existence implies (by Corollary 2.1.14) that $S \rightarrow \mathscr{S}^{\text {an }}$ lands inside of $\mathscr{V}^{\text {an }}$, so analytification of the generalized elliptic curve 
structure over $\mathscr{V}$ gives a generalized elliptic curve over $\mathscr{V}$ an that pulls back to the desired generalized elliptic curve structure on $C_{/ S}$ since $\alpha^{\text {an }}$ pulls back to $\rho$.

The significance of Theorem 2.2.1 (along with Corollary 2.1.14 and Theorem B.2.6 on fibers) is that for any DR semistable genus- 1 curve $C$ over a rigid space $S$, a generalized elliptic curve structure on $C_{/ S}$ may be uniquely constructed in accordance with the following data: a relatively ample relative effective Cartier divisor $D \hookrightarrow C$ (supported in $C^{\mathrm{sm}}$ ), a commutative $S$-group structure on $D$, and an action map $\rho: D \times C \rightarrow C$ such that for all $s \in S$ the algebraized action map $\rho_{s}^{\text {alg }}: D_{s}^{\text {alg }} \times C_{s}^{\text {alg }} \rightarrow C_{s}^{\text {alg }}$ under GAGA over fields makes $D_{s}^{\text {alg }}$ act trivially

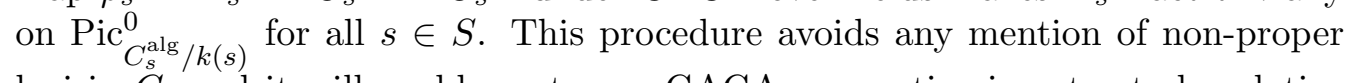
loci in $C$, and it will enable us to use GAGA over artin rings to study relative rigid-analytic generalized elliptic curves. However, this viewpoint is of limited use at present because we do not (yet) know that an arbitrary generalized elliptic curve $E \rightarrow S$ over an arbitrary rigid space $S$ must, locally on $S$, admit a divisor $D$ as in Theorem 2.2.1! In the scheme case $f: \mathscr{E} \rightarrow \mathscr{S}$ over a base scheme $\mathscr{S}$, we can take $\mathscr{D}$ in Theorem B.2.6 (the algebraic version of Theorem 2.2.1) to be a suitable torsion subscheme, with the torsion-level governed by the structure of the locus of non-smoothness $\mathscr{S}^{\infty, f}$ as in Lemma B.2.2. Let us review how this works, as it will motivate our construction of $D$ in the rigid-analytic case (though we shall encounter non-trivial finiteness problems in the rigid-analytic case that do not arise in the algebraic case).

Let $f: \mathscr{E} \rightarrow \mathscr{S}$ be a generalized elliptic curve over a scheme. By the structure of $\mathscr{S}^{\infty, f}$ in Lemma B.2.2, we may cover $\mathscr{S}$ by Zariski-opens $\mathscr{S}_{n}$ so that all nonsmooth geometric fibers of $\mathscr{E}$ over $\mathscr{S}_{n}$ are $n$-gons for some $n \geq 1$. Thus, upon pulling back to $\mathscr{S}_{n}$ and renaming the pullback as $\mathscr{E} \rightarrow \mathscr{S}$ the multiplication map $[n]: \mathscr{E}^{\mathrm{sm}} \rightarrow \mathscr{E}^{\mathrm{sm}}$ is flat, separated, and finitely presented with all fibers of the same rank, namely $n^{2}$. It therefore follows from the valuative criterion for properness and the structure theorem for quasi-finite separated maps that the multiplication map $[n]$ on the smooth locus $\mathscr{E}^{\mathrm{sm}}$ is a proper and hence finite map (see [DR, II, 1.19] for details in the locally noetherian case, which suffices to infer the general case). In this situation $\mathscr{D}=\mathscr{E}^{\mathrm{sm}}[n]$ is finite locally free over $\mathscr{S}$ and is a relatively ample relative effective Cartier divisor in $\mathscr{E}$ over $\mathscr{S}$.

In the rigid-analytic case we are confronted with a new difficulty: even after we prove the rigid-analytic analogue of Lemma B.2.2 (see Theorem 2.2.3), if all non-smooth geometric fibers are $n$-gons for some $n \geq 1$ then the rigid-analytic multiplication map $[n]: E^{\mathrm{sm}} \rightarrow E^{\mathrm{sm}}$ is a priori merely flat and separated with finite fibers that all have rank $n^{2}$. In the analogous scheme setting $[n]$ is automatically quasi-compact, but in the rigid-analytic setting it is not at all obvious from the definitions that $[n]$ is quasi-compact, so we cannot see without effort that $E^{\mathrm{sm}}[n]$ is $S$-finite (and in fact quasi-compactness is the essential obstacle: 
a quasi-compact separated flat map of rigid spaces is finite if and only if it has finite fibers and its fiber-rank is locally constant on the base; see Theorem A.1.2). Thus, we will need to do some more work before we can determine the structure of torsion subgroups in the rigid-analytic case and thereby make Theorem 2.2.1 be useful.

The first step toward analyzing finiteness properties of $[n]$ in the rigid-analytic case is to establish the rigid analogue of Lemma B.2.2. Let $f: E \rightarrow S$ be a generalized elliptic curve over a rigid space $S$, and give the Zariski-closed set $E^{\text {sing }}=E-E^{\mathrm{sm}}$ in $E$ its canonical "Fitting ideal" rigid space structure inside of $E$ (as in the scheme case). Since $\pi: E^{\text {sing }} \rightarrow S$ is proper and quasi-finite, it is finite. In particular, the formation of $\pi_{*} \mathscr{O}_{E^{\text {sing }}}$ commutes with arbitrary base change on $S$.

Definition 2.2.2. Let $f: E \rightarrow S$ be a generalized elliptic curve. The locus of non-smoothness for $f$ is the canonical closed immersion $S^{\infty, f} \hookrightarrow S$ defined by the coherent ideal sheaf $\operatorname{ker}\left(\mathscr{O}_{S} \rightarrow \pi_{*} \mathscr{O}_{E^{\text {sing }}}\right)$.

By fiber calculations we see that the support of the locus of non-smoothness is the set of $s \in S$ such that $E_{s}$ is not smooth. In the scheme case this construction recovers the locus of non-smoothness $S^{\infty, f}$ as the scheme-theoretic image of the non-smooth locus on the curve (see Definition B.1.5). It is obvious that the formation of $S^{\infty, f}$ in the rigid-analytic case is compatible with analytification from the scheme case. Here is the global structure of $S^{\infty, f}$ :

Theorem 2.2.3. The formation of $S^{\infty, f}$ is compatible with base change on $S$. Moreover, the rigid space $S^{\infty, f}$ is a disjoint union of Zariski-opens $S_{n}^{\infty, f} \subseteq S^{\infty, f}$ over which the geometric fibers of $E_{/ S^{\infty, f}}$ are $n$-gons.

Proof. Let $\pi: E^{\operatorname{sing}} \rightarrow S$ denote the finite structure map. The Zariski-closed set $S^{\infty, f}$ is defined by the coherent kernel of $\mathscr{O}_{S} \rightarrow \pi_{*} \mathscr{O}_{E^{\text {sing }}}$, so its formation certainly commutes with finite extension on the ground field. We must prove that formation of $S^{\infty, f}$ commutes with base change on $S$. Since $\mathscr{O}_{S} \rightarrow \pi_{*} \mathscr{O}_{E^{\text {sing }}}$ is a map of coherent sheaves, by consideration of completions at stalks and [EGA, $\left.0_{\mathrm{I}}, 7.2 .9\right]$ we reduce to the case of an artin local base $S=\operatorname{Sp}(B)$ and base change to an artin local $S^{\prime}$ (necessarily finite) over $S$. We may assume that $E_{/ S}$ is nonsmooth, so by Corollary 2.1.13 we may arrange (by replacing $k$ with a sufficiently large finite extension) that the closed fiber is isomorphic to an $n$-gon with its standard generalized elliptic curve structure for some $n \geq 1$. By increasing $k$ some more, we can arrange that the finite local $k$-algebra $B$ has residue field $k$.

The map $[n]: E^{\mathrm{sm}} \rightarrow E^{\mathrm{sm}}$ is flat with finite fibers, due to Corollary A.2.6(1) and the identification of the closed fiber with a standard $n$-gon (as a generalized elliptic curve). On the closed fiber over our artin local base $S=\operatorname{Sp}(B)$ this flat map $[n]$ becomes finite (of degree $n^{2}$ ). By Theorem A.1.1, $[n]$ must therefore be 
a finite map, hence finite flat of degree $n^{2}$. In particular, $D=E^{\mathrm{sm}}[n]$ is a finite flat commutative group object over $S$, and $D$ is an $S$-ample relative effective Cartier divisor in $E_{/ S}$. By GAGA over artin rings, $E$ has a unique "algebraic" incarnation as a DR semistable genus-1 curve $f^{\text {alg }}: E^{\text {alg }} \rightarrow \operatorname{Spec}(B)$ and there is a unique relative effective Cartier divisor $D^{\text {alg }} \hookrightarrow E^{\text {alg }}$ that is finite flat over $\operatorname{Spec}(B)$ and induces $D \hookrightarrow E$ upon analytification. Clearly $D^{\text {alg }}$ is $B$-ample and is supported in the smooth locus of $E_{/ B}^{\text {alg }}$, and there is a unique compatible structure of commutative $B$-group scheme on $D^{\text {alg }}$.

By Theorem 2.2.1, the generalized elliptic curve structure on $E_{/ S}$ is the unique one compatible with the group structure on $D$. By GAGA over artin rings, the analytic action map $\rho^{\text {an }}: D \times E \rightarrow E$ is the analytification of an algebraic action map

$$
\rho^{\mathrm{alg}}: D^{\mathrm{alg}} \times E^{\mathrm{alg}} \rightarrow E^{\mathrm{alg}} .
$$

The proof of uniqueness in Theorem 2.2.1 shows that there is a unique structure of generalized elliptic curve on $E_{/ B}^{\text {alg }}$ compatible with $\rho^{\text {alg }}$, and that this generalized elliptic curve structure on $E_{/ B}^{\text {alg }}$ must analytify to the given generalized elliptic curve structure on $E_{/ \operatorname{Sp}(B)}$. Likewise, $\operatorname{Spec}(B)^{\infty, f^{\text {alg }}}$ must analytify to $\operatorname{Sp}(B)^{\infty, f}$. This is all valid over an arbitrary local artin object over $\operatorname{Sp}(k)$, so applying Theorem B.2.4 for base change between artin local base schemes completes the proof of base change compatibility of $S^{\infty, f}$.

With base change compatibility for $S^{\infty, f}$ now settled in general, we may assume $S=S^{\infty, f}$ and we first want to show that if the geometric fiber over some $s \in$ $S$ is an $n$-gon then the same is true for geometric fibers over a Zariski-open neighborhood of $s$. We will adapt some ideas in the proof of [DR, II, 1.16]. Recall that the formation of $E^{\text {sing }}$ is compatible with base change on $S$. We claim that the formation of the rigid-analytic blow-up

$$
\widetilde{E}=\mathrm{Bl}_{E^{\operatorname{sing}}}(E)
$$

(see [C3, §4.1] for blow-ups in the rigid-analytic case) is compatible with base change on $S$ via the natural map. By the definition of blow-ups, it suffices to check that the infinitesimal neighborhoods of $E^{\text {sing }}$ in $E$ are $S$-flat; to verify such flatness it suffices (by the local flatness criterion [Mat, 22.3]) to work on infinitesimal fibers, so we may suppose that the base is an artin local object $S=\operatorname{Sp}(B)$. By GAGA over artin rings, it suffices to prove $B$-flatness of the infinitesimal neighborhoods of $\left(E^{\text {alg }}\right)^{\text {sing }}$ in $E^{\text {alg }}$, with $f^{\text {alg }}: E^{\text {alg }} \rightarrow \operatorname{Spec}(B)$ a generalized elliptic curve over the local artin $\operatorname{scheme} \operatorname{Spec}(B)$ such that $\operatorname{Spec}(B)=$ $\operatorname{Spec}(B)^{\infty, f^{\text {alg }}}$ (since we are in the case $S=S^{\infty, f}$ ). As is explained in the proof of [DR, II, 1.16], the equality $\operatorname{Spec}(B)=\operatorname{Spec}(B)^{\infty, f^{\text {alg }}}$ ensures that fppf-locally 
on the generalized elliptic curve $E^{\text {alg }}$, the closed immersion

$$
\left(E^{\mathrm{alg}}\right)^{\operatorname{sing}} \hookrightarrow E^{\mathrm{alg}}
$$

looks like $\operatorname{Spec}(B) \hookrightarrow \operatorname{Spec}(B[x, y] /(x y))$. Thus, the $B$-flatness of the infinitesimal neighborhoods of the closed subscheme $(2.2 .3)$ is seen by explicit calculation. We may now compute fibers of the blow-up (2.2.2) by blowing up the fibers.

Using Corollary 2.1.13 and analytification of blow-ups of infinitesimal algebraic fibers, we conclude that

- the fiber of $\widetilde{f}: \widetilde{E}=\mathrm{Bl}_{E^{\text {sing }}}(E) \rightarrow S$ over each $s \in S$ becomes isomorphic to a finite disjoint union of projective lines after passing to a finite extension of $k(s)$,

- the infinitesimal fibers of $\widetilde{f}$ are $S$-flat (so $\widetilde{f}$ is flat).

It follows that $\mathrm{H}^{1}\left(\widetilde{E}_{s}, \mathscr{O}_{\widetilde{E}_{s}}\right)=0$ for the fibers $\widetilde{E}_{s}$ of $\widetilde{f}$, and by $S$-flatness of $\widetilde{f}$ we may use cohomology and base change to conclude that the direct image $\widetilde{f}_{*} \mathscr{O}_{\widetilde{E}}$ is finite flat with formation that is compatible with base change on $S$. Working on fibers shows that the finite flat $\mathscr{O}_{S}$-algebra $\widetilde{f}_{*}\left(\mathscr{O}_{\widetilde{E}}\right)$ is étale and that its rank counts the number of sides on a geometric-fiber polygon for fibers of $f$, so this number is locally constant for the Zariski topology on $S=S^{\infty, f}$. Thus, upon returning to the case of a general base $S$ (where perhaps $S^{\infty, f} \neq S$ ), we conclude that $S^{\infty, f}$ is a disjoint union of Zariski-open loci $S_{n}^{\infty, f}$ over which the geometric fibers of $E$ are $n$-gons. The formation of these $S_{n}^{\infty, f}$ clearly commutes with base change on $S$.

We are now ready to prove the desired finiteness property concerning the flat $\operatorname{map}[d]: E^{\mathrm{sm}} \rightarrow E^{\mathrm{sm}}$.

Theorem 2.2.4. Let $E_{/ S}$ be a generalized elliptic curve over a rigid space $S$ and assume that the number of geometric irreducible components in every non-smooth fiber is divisible by $d \geq 1$. The $\operatorname{map}[d]: E^{\mathrm{sm}} \rightarrow E^{\mathrm{sm}}$ is finite flat of degree $d^{2}$.

Proof. By Theorem 2.2.3, we may work locally on $S$ so as to reduce to the case in which the number of geometric irreducible components in each non-smooth fiber is a common value, say $n$. Since $[n]=[n / d] \circ[d]$ and $[n / d]$ is separated, if $[n]$ is finite then $[d]$ is finite. Thus, we may assume $n=d$. Finally, by $f p q c$-descent for properties of rigid-analytic morphisms [C3, Thm. 4.2.7] we may make an fpqc base change on $S$. Hence, by Theorem 2.1.4 we may assume that there exists a closed immersion $E \hookrightarrow \mathbf{P}_{S}^{N}$. Also, by the Zariski-local constancy of Euler characteristics in rigid-analytic proper flat families (proved by straightforward adaptation of the algebraic methods in [Mum, $\S 5]$ ) we can suppose that the Hilbert polynomials of the fibers are all equal to the same polynomial $\Phi=\delta t \in \mathbf{Q}[t]$ (so $E_{s} \hookrightarrow \mathbf{P}_{s}^{N}$ is a curve of degree $\delta$ for all $s \in S$ ). The idea of the rest of the proof is to use 
Hilbert and Hom functors for both schemes and rigid spaces to show (via Theorem 2.2.1) that $E \rightarrow S$ is étale-locally (on $S$ ) a pullback of the analytification of a generalized elliptic curve $\mathscr{E} \rightarrow \mathscr{S}$ over a locally finite type $k$-scheme $\mathscr{S}$ whose non-smooth geometric fibers are all $d$-gons. The quasi-compact multiplication map $[d]_{\mathscr{E}^{s m}}$ is necessarily finite [DR, II, 1.19], so by analytification and pullback we would obtain the desired finiteness of the flat map $[d]_{E^{\mathrm{sm}}}$. Inspecting fibers then ensures that this finite flat map has constant degree $d^{2}$.

The fiber of $E$ over each $s \in S^{\infty, f}$ becomes isomorphic to the standard $d$-gon over a finite extension of $k(s)$, by Corollary 2.1.13. We want to "interpolate" generators of geometric component groups of fibers of $E_{/ S}^{\mathrm{sm}}$. More precisely, we claim that there exists

- a Zariski-open covering $\left\{S_{i}\right\}$ of $S$,

- a finite étale cover $S_{i}^{\prime} \rightarrow S_{i}$,

- sections $g_{1, i}^{\prime}, \ldots, g_{\delta, i}^{\prime} \in E^{\mathrm{sm}}\left(S_{i}^{\prime}\right)$

such that on each fiber $E_{s^{\prime}}$ of $E$ over $s^{\prime} \in S_{i}^{\prime}$ at least one of the points $g_{j, i}^{\prime}\left(s^{\prime}\right)$ induces a generator of the geometric component group of the smooth locus $E_{s^{\prime}}^{\mathrm{sm}}$. Since we can take one of the Zariski-opens to be $S-S^{\infty, f}$, over which the identity section $e \in E^{\mathrm{sm}}(S)$ generates the geometric component group of every fiber, to carry out the construction we just have to find $S_{i}$ 's and $S_{i}^{\prime}$ 's of the above type such that the Zariski-open $S_{i}$ 's in $S$ cover the Zariski-closed set $S^{\infty, f}$ set-theoretically.

Let us begin the construction by choosing $s \in S^{\infty, f}$. We claim that there exists a Zariski-open $V$ around $s$ in $S$ and a relative hyperplane $H \hookrightarrow \mathbf{P}_{V}^{N}$ such that the map $H \cap E_{V} \rightarrow V$ is finite étale surjective and is supported in $E_{V}^{\mathrm{sm}}$. Once such $V$ and $H$ are constructed, a standard fiber product construction yields a finite étale cover $V^{\prime} \rightarrow V$ that splits the degree- $\delta$ finite étale cover $H \cap E_{V} \rightarrow V$, and so if $g_{1}, \ldots, g_{\delta} \in\left(H \cap E_{V}\right)\left(V^{\prime}\right)$ are pairwise disjoint sections that define a splitting then by ampleness of hyperplane sections over fields it would follow that for each fiber $E_{v^{\prime}}^{\mathrm{sm}}$ of $E^{\mathrm{sm}}$ over $V^{\prime}$ at least one $g_{j}\left(v^{\prime}\right)$ is a generator of the geometric component group of $E_{v^{\prime}}^{\mathrm{sm}}$.

We now construct such $V$ and $H$. By Lemma B.1.3 and the compatibility of representing objects for the algebraic and rigid-analytic Hilbert functors [C3, Thm. 4.1.3], the closed immersion $E \hookrightarrow \mathbf{P}_{S}^{N}$ is the pullback of a DR semistable genus-1 curve $\mathscr{C} \hookrightarrow \mathbf{P}_{\mathscr{S}}^{N}$ with fibral degree $\delta$ over a locally finite type $k$-scheme $\mathscr{S}$. Thus, it suffices to solve a universal algebraic problem: if $\mathscr{C} \hookrightarrow \mathbf{P}_{\mathscr{S}}^{N}$ is a closed immersion with $\mathscr{C}$ a DR semistable genus-1 curve over a scheme $\mathscr{S}$, and $s \in \mathscr{S}$ is a point with infinite residue field, we seek a Zariski-open neighborhood $\mathscr{V}$ of $s$ and a relative hyperplane $\mathscr{H} \hookrightarrow \mathbf{P}_{\mathscr{V}}^{N}$ such that $\mathscr{H} \cap \mathscr{C}_{\mathscr{V}}$ is finite étale over $\mathscr{V}$ and is supported in $\mathscr{C}^{\text {sm }}$. We can assume $\mathscr{S}$ is local noetherian with $s$ the closed point. By Bertini's theorem, the scheme $U$ of hyperplanes in $\mathbf{P}_{\operatorname{Spec} k(s)}^{N}$ 
whose intersection with $\mathscr{C}_{s}$ is étale and disjoint from the finite $\mathscr{C}_{s}-\mathscr{C}_{s}^{\text {sm }}$ is a dense open in the dual projective space. Since $k(s)$ is assumed to be infinite, the set $U(k(s))$ is non-empty. Let $H \subseteq \mathbf{P}_{\text {Spec } k(s)}^{N}$ be a hyperplane corresponding to a point in $U(k(s))$. By lifting the coefficients of a defining equation, we get a relative hyperplane $\mathscr{H}$ in $\mathbf{P}_{\mathscr{S}}^{N}$ over the local scheme $\mathscr{S}$ and we claim that this has the desired properties.

Certainly $\mathscr{H} \cap \mathscr{C} \subseteq \mathscr{C}^{\text {sm }}$, since the non-smooth locus of $\mathscr{C}$ is proper over the local $\mathscr{S}$ and its closed fiber is disjoint from $\mathscr{H}$ (by the choice of $\mathscr{H}_{s}=H \subseteq$ $\left.\mathbf{P}_{\text {Spec } k(s)}^{N}\right)$. Also, the proper map $\mathscr{H} \cap \mathscr{C} \rightarrow \mathscr{S}$ to the local base $\mathscr{S}$ has finite closed fiber, so it must be quasi-finite (by openness of the quasi-finite locus [EGA, $\left.\left.\mathrm{IV}_{3}, 13.1 .4\right]\right)$ and hence finite. Since $\mathscr{S}$ is local noetherian, to verify that the $\mathscr{S}$ finite $\mathscr{H} \cap \mathscr{C}$ is étale over $\mathscr{S}$ it suffices to treat the case when the local $\mathscr{S}$ is artin local.

Now assume $\mathscr{S}$ is artin local. Choose one of the finitely many points $x$ in $\mathscr{H} \cap \mathscr{C}$ and let $t \in \mathscr{O}_{\mathscr{C}, x}$ be the pullback of a defining equation in $\mathscr{O}_{\mathbf{P}_{\mathrm{s}^{\prime}}^{N}, x}$ for the hyperplane $\mathscr{H}$. We have to show that the finite map $\operatorname{Spec}\left(\mathscr{O}_{\mathscr{C}, x} /(t)\right) \rightarrow \mathscr{S}$ is étale. Let $U$ be an open neighborhood of $x$ in $\mathscr{C}^{\text {sm }}$ over which $t$ smears out to an $\mathscr{S}$-map $t: U \rightarrow \mathbf{A}_{\mathscr{S}}^{1}$. This map physically sends $x$ to the origin at the level of fibers over $s$. The map $t_{s}$ on $s$-fibers is a map between smooth curves over $k(s)$, so it is étale at $x$ since $\mathscr{H}_{s} \cap \mathscr{C}_{s}$ is étale at $x$. By shrinking $U$, we can therefore assume that $t_{s}$ is étale, so $t$ is flat (by the fiber-by-fiber criterion for flatness over the artin local $\mathscr{S})$ and hence $t$ is étale at $x$. The fiber $t^{-1}(0)$ over the zero-section of $\mathbf{A}_{\mathscr{S}}^{1}$ is then quasi-finite, flat, and separated over $\mathscr{S}$ and étale at $x$. But $\mathscr{S}$ is artinian, so the connected component of $t^{-1}(0)$ through $x$ is finite étale over $\mathscr{S}$. That is, the map $\operatorname{Spec}\left(\mathscr{O}_{\mathscr{C}, x} /(t)\right) \rightarrow \mathscr{S}$ is finite étale. This completes the construction of the desired Zariski-opens $S_{i}$ in our original rigid space $S$, along with the finite étale covers $S_{i}^{\prime} \rightarrow S_{i}$ and sections $g_{i j}^{\prime} \in E^{\mathrm{sm}}\left(S_{i}^{\prime}\right)$.

By the theory of $f p q c$ descent for properties of rigid-analytic morphisms [C3, Thm. 4.2.7] we may rename $S_{i}^{\prime}$ as $S$, so we may assume that there exist sections $g_{1}, \ldots, g_{\delta} \in E^{\mathrm{sm}}(S)$ such that on each fiber $E_{s}^{\mathrm{sm}}$ at least one of the $g_{j}(s)$ 's is a generator of the geometric component group. By the Zariski-openness of the locus $U_{j}$ of $s \in S$ such that the $d$ multiples $\left\{0(s), g_{j}(s), \ldots,\left([d-1]\left(g_{j}\right)\right)(s)\right\}$ of $g_{j}(s)$ are pairwise distinct, we may finally assume that there exists $g \in E^{\mathrm{sm}}(S)$ that induces a generator of every (geometric) fibral component group. That is, the relative effective Cartier divisor $\sum_{0 \leq j \leq d-1}[j](g)$ is $S$-ample.

The fibral criterion for relative ampleness in the scheme case [EGA, $\mathrm{IV}_{4}, 9.6 .4$ ] and the openness of the DR semistable genus- 1 locus for a proper relative curve over a scheme (Lemma B.1.3) enable us to use Hilbert schemes and Hom-schemes to construct a DR semistable genus-1 curve $\mathscr{C} \rightarrow \mathscr{S}$ over a (locally finite type) $k$-scheme $\mathscr{S}$ such that $\mathscr{C} \rightarrow \mathscr{S}$ is universal for being equipped with (i) a closed 
immersion into $\mathbf{P}_{\mathscr{S}}^{N}$, (ii) a section $e \in \mathscr{C}^{\mathrm{sm}}(\mathscr{S})$, and (iii) an $\mathscr{S}$-automorphism $t: \mathscr{C} \rightarrow \mathscr{C}$ such that $\sum_{0 \leq j<d} t^{j}(e)$ is relatively ample. By the rigid-analytic theory of Hilbert and Hom functors [C3, Thm. 4.1.3, Cor. 4.1.5], the analytification of this universal algebraic data over Spec $k$ is universal in the category of rigid spaces over $\operatorname{Sp}(k)$. Thus, $\left(E \hookrightarrow \mathbf{P}^{N}, e, x \mapsto g+x\right)_{/ S}$ is the pullback of the universal algebraic data $\left(\mathscr{C} \hookrightarrow \mathbf{P}_{\mathscr{S}}^{N}, e, t\right)$ by a unique $k$-morphism $\xi: S \rightarrow \mathscr{S}$.

For each $s \in S$, consider the induced morphism $t_{\xi(s)}: \mathscr{C}_{\xi(s)} \rightarrow \mathscr{C}_{\xi(s)}$. By Theorem 2.2.1 and the GAGA comparison over fields between $k(s) \otimes_{k(\xi(s))} \mathscr{C}_{\xi(s)}$ and $E_{s}$, the map $t_{\xi(s)}$ acts transitively on the geometric connected components of $\mathscr{C}_{\xi(s)}^{\mathrm{sm}}$ and (by Lemma B.2.7) there is a universal Zariski-open neighborhood of $\xi(s)$ in $\mathscr{S}$ over which $t$ restricts to the identity on the semi-abelian algebraic space group $\operatorname{Pic}_{\mathscr{C} / \mathscr{S}}^{0}$. Thus, as at the end of the proof of Theorem 2.2.1, it follows from Theorem B.2.5 (with the constant group scheme $G=\mathbf{Z}$, as opposed to the special case in Theorem B.2.6) and Lemma B.2.7 that after replacing $\mathscr{S}$ with a canonical Zariski-open around the image of $S$ there exists a unique structure of generalized elliptic curve on $\mathscr{C} / \mathscr{S}$ with identity section $e$ such that $t$ acts as translation by $t(e) \in \mathscr{C}^{\mathrm{sm}}(\mathscr{S})$.

The pullback of this generalized elliptic curve structure to the bare curve $E_{/ S}$ must be the initial generalized elliptic curve structure on $E_{/ S}$ that we had at the outset! Indeed, by Lemma 2.1.12 and Theorem 2.2.1 this follows by applying GAGA over artin rings to infinitesimal fibers and applying the auxiliary fact (Theorem B.2.5) that any $\mathscr{S}$-automorphism of $\mathscr{C}$ commuting with $t$ must be a translation. We have now realized the generalized elliptic curve $E$ over the rigid space $S$ as the pullback of a generalized elliptic curve $\mathscr{E}$ over a locally finite type $k$-scheme $\mathscr{S}$. Since $S \rightarrow \mathscr{S}$ must factor through the Zariski-open locus $\mathscr{U} \subseteq \mathscr{S}$ over which $\mathscr{E}$ has smooth or $d$-gon geometric fibers, the finiteness of $[d]$ on $E^{\mathrm{sm}}$ follows from the finiteness of $[d]$ on $\left.\mathscr{E}^{\mathrm{sm}}\right|_{\mathscr{U}}$.

The étale-local nature of the construction of $\mathscr{E} \rightarrow \mathscr{S}$ in the preceding proof can now be improved:

Corollary 2.2.5. Let $f: E \rightarrow S$ be a generalized elliptic curve over a rigid space $S$ over $k$. There exists an admissible open covering $\left\{S_{i}\right\}$ of $S$ and generalized elliptic curves $\mathscr{E}_{i / \mathscr{S}_{i}}$ over locally finite type $k$-schemes $\mathscr{S}_{i}$ such that $E_{/ S_{i}}$ is the pullback of $\mathscr{E}_{i / \mathscr{S}_{i}}$ under a k-morphism $S_{i} \rightarrow \mathscr{S}_{i}$.

This corollary says that generalized elliptic curves over rigid spaces are always "locally algebraic", including their group laws.

Proof. Working Zariski-locally on $S$, we may assume (by Theorem 2.2.3) that all non-smooth geometric fibers are $d$-gons for a fixed $d \geq 1$. Thus, by Theorem 2.2.4, $[d]: E^{\mathrm{sm}} \rightarrow E^{\mathrm{sm}}$ is a finite flat map of degree $d^{2}$. Let $D=E^{\mathrm{sm}}[d]$, so $D$ 
is a finite flat commutative $S$-group of order $d^{2}$. We may view $D$ as an $S$-ample relative effective Cartier divisor in $E$. By Theorem 2.2.1, the generalized elliptic curve structure on $E_{/ S}$ is the unique one compatible with the group structure on $D_{/ S}$ and the action map $\rho: D \times E \rightarrow E$.

By the rigid-analytic version of Theorem B.1.2, we have a canonical closed immersion $E \hookrightarrow \mathbf{P}\left(f_{*} \mathscr{O}(3 D)\right)$ into a $\mathbf{P}^{3 d^{2}-1}$-bundle (for the Tate topology) and the formation of this map is compatible with base change on $S$. Working locally on $S$, we may assume that the coherent locally free $\mathscr{O}_{S}$-module $f_{*} \mathscr{O}(3 D)$ is globally free, so we have a closed immersion $E \hookrightarrow \mathbf{P}_{S}^{3 d^{2}-1}$ over $S$.

As in the proof of Theorem 2.2.1, there exists a locally finite type $k$-scheme $\mathscr{S}$ and a DM semistable genus-1 curve $\mathscr{C} \hookrightarrow \mathbf{P}_{\mathscr{S}}^{3 d^{2}-1}$ and a degree- $d^{2}$ relative effective Cartier divisor $\mathscr{D} \hookrightarrow \mathscr{C}$ supported in $\mathscr{C}^{\mathrm{sm}}$ such that under a $k$-morphism $\varphi: S \rightarrow \mathscr{S}$ the data $\mathscr{D} \hookrightarrow \mathscr{C} \hookrightarrow \mathbf{P}_{\mathscr{S}}^{3 d^{2}-1}$ pulls back to

$$
D \hookrightarrow E \hookrightarrow \mathbf{P}_{S}^{3 d^{2}-1} .
$$

Moreover, we can likewise arrange that $\mathscr{D}$ is endowed with a commutative $\mathscr{S}$ group structure extending to an action $\rho^{\text {alg }}$ on $\mathscr{C}$ such that $\varphi$ is compatible with the group structures on $\mathscr{D}$ and $D$ as well as with the action maps $\rho^{\text {alg }}$ and $\rho$.

Choose $s \in S$ and let $s^{\prime}=\varphi(s) \in \mathscr{S}$ be the closed-point image. By Corollary 2.1.13 and Theorem B.2.6, $\mathscr{D}_{s^{\prime}}$ acts trivially on the semi-abelian variety $\mathrm{Pic}_{\mathscr{C}_{s^{\prime}} / s^{\prime}}^{0}$ (as it suffices to check this on a geometric fiber over $s^{\prime}$ ). By Theorem B.2.6, there is a Zariski-open and Zariski-closed locus $\mathscr{V}$ in $\mathscr{S}$ that represents the condition that $\mathscr{D} / \mathscr{S}^{\prime}$ acts trivially on $\operatorname{Pic}_{\mathscr{C}^{\prime} / \mathscr{S}^{\prime}}^{0}$ (for variable $\mathscr{S}$-schemes $\mathscr{S}^{\prime}$ ). In particular, $s^{\prime} \in \mathscr{V}$. Thus, $S \rightarrow \mathscr{S}$ factors through $\mathscr{V} \subseteq \mathscr{S}$. Replacing $\mathscr{S}$ with $\mathscr{V}$, we may assume that $\mathscr{D}$ acts trivially on $\operatorname{Pic}_{\mathscr{C} / \mathscr{S}}^{0}$.

Now we can use Theorem B.2.6 to conclude that there is a unique structure of generalized elliptic curve on $\mathscr{C}_{/ \mathscr{S}}$ compatible with the group structure on $\mathscr{D}$ and the action of $\mathscr{D}$ on $\mathscr{C}$. By analytifying this structure and using Theorem 2.2.1, it follows that the generalized elliptic curve structure on $\mathscr{C}_{/ \mathscr{S}}$ pulls back to the generalized elliptic curve structure on $E_{/ S}$.

Corollary 2.2.6. Let $E \rightarrow S$ be a generalized elliptic curve over a rigid space. The inversion morphism on the group object $E^{\mathrm{sm}}$ uniquely extends to an $S$ morphism $E \rightarrow E$, and this morphism is an involution of the generalized elliptic curve $E_{/ S}$.

Proof. Granting existence, for the rest we can work on infinitesimal fibers and thereby use Theorem 2.2.1, Theorem B.2.6, and GAGA over artin rings to deduce everything from the algebraic case [DR, II, 2.8]. In particular, we have uniqueness in general and so may work locally for existence. Corollary 2.2.5 reduces existence to the known algebraic case. 
Corollary 2.2.7. Let $(E \rightarrow S,+, e)$ be a generalized elliptic curve over a quasiseparated or pseudo-separated rigid space over $k$. For any analytic extension field $k^{\prime} / k,\left(E^{\prime} \rightarrow S^{\prime},+^{\prime}, e^{\prime}\right)$ is a generalized elliptic curve over $S^{\prime}$. Moreover, the closed immersions $S^{\infty, f} \hookrightarrow S$ and $S^{\prime \infty, f^{\prime}} \hookrightarrow S^{\prime}$ defining the loci of non-smoothness satisfy

$$
k^{\prime} \widehat{\otimes}_{k} S^{\infty, f}=S^{\prime \infty, f^{\prime}}
$$

inside of $S^{\prime}$; the same holds for the loci $S_{n}^{\infty, f}$ and ${S^{\prime}}_{n}^{\infty, f^{\prime}}$ of n-gon geometric fibers for each $n \geq 1$.

Proof. We may work locally on $S$, so by Corollary 2.2 .5 we can assume that $(E,+, e)$ is the pullback of a generalized elliptic curve $\mathscr{E}$ over a $k$-scheme $\mathscr{S}$ that is locally of finite type. By Theorem 2.2.3, the formation of the rigid spaces $S^{\infty, f}$ and $S_{n}^{\infty, f}$ commutes with arbitrary rigid-analytic base change (for a fixed ground field), so working with infinitesimal fibers shows that these rigid spaces inside $S$ must be the pullbacks of the corresponding closed subschemes of $\mathscr{S}$ associated to $\mathscr{E}$. Hence, by the compatibility of the analytification and change of base field functors, our analytic problems are reduced to the trivial algebraic case.

\section{UNIVERSAL STRUCTURES}

3.1. Relative rigid Tate curves and a variant on Berthelot's functor. For applications in the remainder of this paper, we need to construct an important class of generalized elliptic curves over an open unit disc over a non-archimedean field, the relative Tate curves. Our approach is based on a variant of Berthelot's general analytification functor from formal schemes to rigid spaces. (For applications to Tate curves it is surely possible to proceed in a less abstract manner, but for the needs of [C4] we prefer to use methods applicable in higher-dimensional cases.) The construction of Berthelot's functor in [deJ, §7] requires discreteness of the valuation, due to the use of noetherian hypotheses, so we will follow another functorial technique (in the spirit of the initial discussion in [deJ, 7.1]) that allows us to avoid discreteness restrictions on the absolute value. In practice the discretely-valued case is the most important case, but we allow arbitrary $k$ throughout.

We refer the reader to $[\mathrm{C} 2, \S 2.5]$ for a self-contained discussion of the construction of Tate curves over $\mathbf{Z}$ via formal schemes (done much more generally in [DR, VII]). To set notation that is used below, we now quickly summarize the wellknown conclusions of this algebraic construction. The proper flat formal $n$-gon Tate curve $\widehat{\text { Tate }}_{n}$ over $\operatorname{Spf}\left(\mathbf{Z} \llbracket q^{1 / n} \rrbracket\right)$ is constructed as a gluing of $n$ formal annuli (with a self-gluing in the case $n=1$ ), and its reduction modulo $q^{1 / n}=0$ is the standard $n$-gon over Spec $\mathbf{Z}$. There is a canonical closed immersion of the finite constant formal scheme $\mathbf{Z} / n \mathbf{Z}$ over $\operatorname{Spf}\left(\mathbf{Z} \llbracket q^{1 / n} \rrbracket\right)$ into ${\underline{\widehat{\operatorname{tate}}_{n}}}_{n}$ lifting the canonical 
closed immersion of $\mathbf{Z} / n \mathbf{Z}$ into the smooth locus $\mathbf{G}_{m} \times(\mathbf{Z} / n \mathbf{Z})$ of the standard $n$-gon over Spec $\mathbf{Z}$, and this extends to a "formal rotation" action

$$
(\mathbf{Z} / n \mathbf{Z}) \times{\underline{\text { Tate }_{n}}}_{n} \rightarrow{\underline{\text { Tate }_{n}}}_{n}
$$

over $\operatorname{Spf}\left(\mathbf{Z} \llbracket q^{1 / n} \rrbracket\right)$ that extends the formal group law on $\mathbf{Z} / n \mathbf{Z}$ and lifts the standard rotation action given by the standard generalized elliptic curve structure on the standard $n$-gon.

The algebraization of the formal rotation action (3.1.1) uniquely extends to a generalized elliptic curve structure on the proper flat curve Tate $_{n}$ that algebraizes $\widehat{\text { Tate }}_{n}$. This procedure also provides a canonical isomorphism of $\mathbf{Z} \llbracket q^{1 / n} \rrbracket$-groups

$$
\underline{\text { Tate }}_{n}^{\mathrm{sm}}[n] \simeq \mu_{n} \times \mathbf{Z} / n \mathbf{Z}
$$

lifting the canonical isomorphism on the standard $n$-gon fiber over $q^{1 / n}=0$. For any adic noetherian $\operatorname{ring} A$ and topologically nilpotent element $a \in A$, we write $\widehat{\operatorname{Tate}}_{n}(a)$ (with $A$ understood from context) to denote the base change by the map $\mathbf{Z} \llbracket q^{1 / n} \rrbracket \rightarrow A$ sending $q^{1 / n}$ to $a$, and $\underline{\text { Tate }}_{n}(a)$ denotes its algebraization over $\operatorname{Spec} A$. This algebraization is the base change of Tate $_{n}$ by Spec $A \rightarrow \operatorname{Spec} \mathbf{Z} \llbracket q^{1 / n} \rrbracket$, and as over $\mathbf{Z} \llbracket q^{1 / n} \rrbracket$ this has a unique structure of generalized elliptic curve respecting the $\mathbf{Z} / n \mathbf{Z}$-action. We wish to functorially "analytify" Tate ${ }_{n}$, or rather $\widehat{\text { Tate }}_{n}$, over $k$ to obtain a rigid analytic generalized elliptic curve Tate ${ }_{n}^{\text {an }}$ over the open unit disc $\Delta=\Delta_{/ k}=\{|t|<1\}$ such that the fiber of Tate $_{n}^{\text {an }}$ over any $q_{0} \in \Delta(k)-\{0\}$ is $\mathbf{G}_{m}^{\text {an }} / q_{0}^{n \mathbf{Z}}$. More generally, we want to define an analytification functor from the category of topologically finite type formal $\mathbf{Z} \llbracket q^{1 / n} \rrbracket$-schemes to the category of rigid spaces over $k$. In fact, we wish to define such a functor on a wider class of formal schemes:

Definition 3.1.1. Let $C$ be an adic noetherian ring. The category $\mathrm{FS}_{C}$ is the category of locally noetherian formal schemes $\mathfrak{X}$ over $\operatorname{Spf}(C)$ whose underlying reduced ordinary scheme is locally of finite type over the quotient $C_{\text {red }}$ of $C$ by the ideal of topologically nilpotent elements.

Put another way, open affines in an object $\mathfrak{X}$ of $\mathrm{FS}_{C}$ have adic affine algebras that are quotients of rings of the form $\left(C \llbracket q_{1}, \ldots, q_{n} \rrbracket\right)\left\{\left\{T_{1}, \ldots, T_{m}\right\}\right\} \simeq$ $\left(C\left\{\left\{T_{1}, \ldots, T_{m}\right\}\right\}\right) \llbracket q_{1}, \ldots, q_{n} \rrbracket$ with the $(I, q)$-adic topology, where $I$ is an ideal of definition for $C$ and the ring $C \llbracket q \rrbracket$ is considered as an adic ring with the $(I, q)$-adic topology when forming restricted power series in the $T_{j}$ 's over $C \llbracket q \rrbracket$. In both the category $\mathrm{FS}_{C}$ and the category of rigid spaces over $k$ we have a notion of "locally topologically nilpotent" section of the structure sheaf, coinciding with "topologically nilpotent" for sections over open affines/affinoids. The case $C=\mathbf{Z}$ with the discrete topology will be used for analytification of $\widehat{\text { Tate }}_{n}$ 's. 
We now suppose that there is given a continuous map of topological rings $C \rightarrow R$, where $R$ is the valuation ring of $k$ (so the ideals of definition of $C$ map into ideals of definition of $R$ since $C$ is noetherian).

Example 3.1.2. If $k$ is discretely valued, we can take $C=R$. In general, by separately treating the cases when $R$ has mixed characteristic and when $R$ is equicharacteristic we may always find a complete discrete valuation ring $R_{0} \subseteq R$ such that $R_{0} \rightarrow R$ is local. We may then take $C=R_{0}$ with the maximal-adic topology. Note that we can also take $C=R_{0}^{\text {disc }}$ to be the ring $R_{0}$ considered with the discrete topology.

Example 3.1.3. The discrete $\operatorname{ring} C=\mathbf{Z}$ equipped with its unique map to $R$ is a valid choice.

Definition 3.1.4. For any $\mathfrak{X}$ in $\mathrm{FS}_{C}$ and any rigid space $X$ over $k, \operatorname{Hom}_{C}^{\text {cont }}(X, \mathfrak{X})$ is the set of morphisms $f: X \rightarrow \mathfrak{X}$ of locally ringed Grothendieck-topologized spaces (in the sense of [C3, Def. 2.2.2]) such that

- $f^{\sharp}: \mathscr{O}_{\mathfrak{X}} \rightarrow f_{*} \mathscr{O}_{X}$ is a map of sheaves of $C$-algebras (using $C \rightarrow R \subseteq k$ ) and it factors through $f_{*} \mathscr{O}_{X}^{b}$, where $\mathscr{O}_{X}^{b} \subseteq \mathscr{O}_{X}$ is the subsheaf of locally power-bounded sections,

- $f^{\sharp}: \mathscr{O}_{\mathfrak{X}}(\mathfrak{U}) \rightarrow \mathscr{O}_{X}\left(f^{-1}(\mathfrak{U})\right)$ carries locally topologically nilpotent sections to locally topologically nilpotent sections for all open $\mathfrak{U} \subseteq \mathfrak{X}$.

In an evident manner, $\operatorname{Hom}_{C}^{\text {cont }}(X, \mathfrak{X})$ is functorial in both $X$ and $\mathfrak{X}$.

Theorem 3.1.5. Fix an adic noetherian ring $C$ and continuous map $C \rightarrow R$ to the valuation ring of $k$. For any $\mathfrak{X}$ in $\mathrm{FS}_{C}$, the functor $\operatorname{Hom}_{C}^{\text {cont }}(\cdot, \mathfrak{X})$ on rigid spaces over $k$ is represented by a quasi-separated rigid space $\mathfrak{X}^{\text {rig }}$, also denoted $\mathfrak{X}_{/ k}^{\text {rig }}$.

The functor $(\cdot)^{\mathrm{rig}}$ takes open/closed immersions to open/closed immersions, open covers to admissible open covers, and enjoys the following additional properties:

(a) $(\cdot)^{\text {rig }}$ is compatible with the formation of fiber products,

(b) if $k^{\prime} / k$ is an analytic extension field, then there is a unique way

to define isomorphisms

$$
k^{\prime} \widehat{\otimes}_{k} \mathfrak{X}_{/ k}^{\text {rig }} \simeq \mathfrak{X}_{/ k^{\prime}}^{\text {rig }}
$$

that are functorial in $\mathfrak{X}$ and recover the identification $k^{\prime} \widehat{\otimes}_{k} \mathbf{B}_{k}^{1}=$ $\mathbf{B}_{k^{\prime}}^{1}$ for $\mathfrak{X}=\operatorname{Spf} C\{\{T\}\}$,

(c) if $C^{\prime}$ is another such adic noetherian ring endowed with a continuous map $C^{\prime} \rightarrow R$ and there is given a continuous map $C \rightarrow C^{\prime}$ compatible with the maps to $R$, then there are unique functorial 


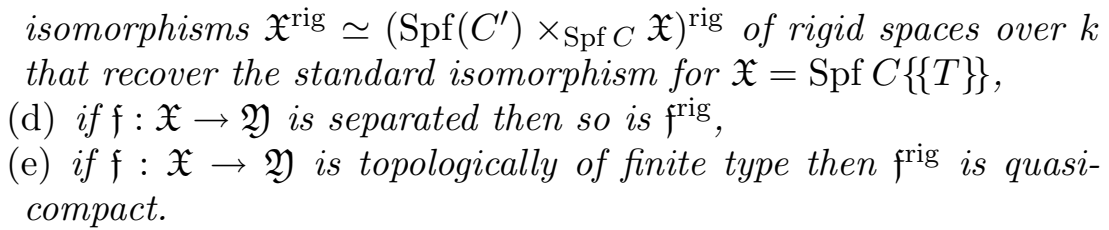

(d) if $\mathfrak{f}: \mathfrak{X} \rightarrow \mathfrak{Y}$ is separated then so is $\mathfrak{f}^{\text {rig }}$,

(e) if $\mathfrak{f}: \mathfrak{X} \rightarrow \mathfrak{Y}$ is topologically of finite type then frig $^{\text {is } q u a s i-}$ compact.

In the special case that $k$ is discretely-valued and $C=R$, this theorem and the next one recover [deJ, 7.2.4, 7.2.6] and also address additional properties concerning flatness, fiber dimension, and properness.

Proof. There is obviously a unique $C$-morphism (in the sense of Definition 3.1.4)

$$
\Delta \rightarrow \operatorname{Spf}(C \llbracket q \rrbracket)
$$

that pulls $q$ back to the coordinate function on $\Delta$; on topological spaces this is the map $\Delta \rightarrow \operatorname{Spec}(k) \rightarrow \operatorname{Spec}\left(C_{\text {red }}\right)$. The map (3.1.3) serves as $\operatorname{Spf}(C \llbracket q \rrbracket)^{\text {rig }}$ because (i) $\Delta$ has an admissible covering given by the discs $\Delta_{r}=\{|t| \leq r\}$ for $r \in(0,1) \cap \sqrt{ }\left|k^{\times}\right|$, and (ii) for any $k$-affinoid algebra $A$ and any $a \in A$ that is topologically nilpotent, we must have $|a|_{\text {sup }}<1$ (i.e., $a: \operatorname{Sp}(A) \rightarrow \mathbf{A}_{k}^{1}$ factors through some $\Delta_{r}$ ). It follows in the general affine case that if $\mathfrak{X} \simeq$ $\operatorname{Spf}\left(C \llbracket q_{1}, \ldots, q_{n} \rrbracket\left\{\left\{T_{1}, \ldots, T_{m}\right\}\right\} / J\right)$ then the zero-space in $\Delta^{n} \times \mathbf{B}^{m}$ defined by the coherent ideal sheaf generated by $J$ serves as $\mathfrak{X}^{\text {rig }}$ (here we are using the "power bounded" condition in the definition of $\left.\operatorname{Hom}_{C}^{\text {cont }}(\cdot, \mathfrak{X})\right)$.

From the construction, if $\mathfrak{X}=\operatorname{Spf}(A)$ is affine and $a \in A$, then $\operatorname{Spf}\left(A_{\{a\}}\right)^{\text {rig }} \rightarrow$ $\mathfrak{X}^{\text {rig }}$ is an isomorphism onto the admissible open $U \subseteq X$ where $a$ is a unit with power-bounded inverse, due to the isomorphism $A_{\{a\}} \simeq A\{\{T\}\} /(1-a T)$. It follows that open/closed immersions of formal affines are taken under $(\cdot)^{\text {rig }}$ to quasi-compact open/closed immersions of rigid spaces. It is therefore clear that for formal affines all of the desired properties hold. In particular, from fiberproduct compatibility we see that $(\cdot)^{\text {rig }}$ is compatible with the formation of overlaps of open affnes within a formal affine $\mathfrak{X}$. Consequently we can construct $\mathfrak{X}^{\text {rig }}$ first for separated $\mathfrak{X}$, and then for general $\mathfrak{X}$, such that it represents the desired functor and such that the formation of $\mathfrak{X}^{\text {rig }}$ is well-behaved with respect to open immersions, closed immersions, and open covers.

Properties (a), (b), and (c) in general follow from the construction, with the uniqueness characterizations in (b) and (c) deduced from the compatibility with localization, closed immersions, and fiber products. The compatibility with separatedness follows from compatibility with fiber products and closed immersions, and by construction we see that if $\mathfrak{f}$ is topologically of finite type then $\mathfrak{f}^{\text {rig }}$ is quasi-compact.

Theorem 3.1.6. With notation as in Theorem 3.1.5, the functor $(\cdot)^{\text {rig }}$ satisfies the following properties for maps $\mathfrak{f}: \mathfrak{X} \rightarrow \mathfrak{Y}$ that are locally topologically of finite type: 
(a) if $\mathfrak{f}$ is finite then so is $\mathfrak{f}^{\mathrm{rig}}$,

(b) if $\mathfrak{f}: \mathfrak{X} \rightarrow \mathfrak{Y}$ is formally smooth (in the category of formal schemes over $\mathfrak{Y})$ with pure relative dimension $d$, then frig is smooth with pure relative dimension $d$; in particular, if $\mathfrak{f}$ is formally étale then $\mathrm{f}^{\text {rig }}$ is étale,

(c) if $\mathfrak{f}$ is finite flat of constant rank $d$ then so is $\mathfrak{f}^{\mathrm{rig}}$,

(d) if $\mathfrak{f}$ is proper then so is $\mathrm{f}^{\mathrm{rig}}$,

(e) if $\mathfrak{f}$ is flat then $\mathfrak{f}^{\text {rig }}$ is flat; the same holds with "flat" replaced by "faithfully flat",

(f) if all fibers of $\mathfrak{f}$ have dimension $\leq d$ then the same holds for frig,

(g) if $\mathfrak{f}$ is flat with geometrically reduced fibers of pure dimension $d$ then the same holds for $\mathrm{f}^{\mathrm{rig}}$.

Remark 3.1.7. Formal smoothness is defined via the usual lifting property with respect to infinitesimal thickenings in the category of locally noetherian formal schemes over $\mathfrak{Y}$.

Proof. For a map $\mathfrak{f}$ in $\mathrm{FS}_{C}$ that is locally topologically of finite type, $\mathfrak{f}$ is formally smooth if and only if it can locally be expressed as formally étale (of topologically finite type) over a formal affine space $\mathfrak{Y}\left\{\left\{T_{1}, \ldots, T_{d}\right\}\right\}$, in which case $d$ is the rank of the coherent locally free $\mathscr{O}_{\mathfrak{X}}$-module $\widehat{\Omega}_{\mathfrak{X} / \mathfrak{Y}}^{1}$. This description is established by using the structure theorems for smooth and étale maps, as well as the insensitivity of the étale site with respect to nilpotent thickenings [EGA, $\left.\mathrm{IV}_{4}, 17.11 .4,18.4 .5(i i), 18.1 .2\right]$. Since $(\cdot)^{\text {rig }}$ behaves well with respect to open immersions, (b) is reduced to the trivial case $\mathfrak{X}=\operatorname{Spf}(\mathscr{B})$ and $\mathfrak{Y}=\operatorname{Spf}(\mathscr{A})$ with

$$
\mathscr{B}=\mathscr{A}\left\{\left\{T_{1}, \ldots, T_{d}\right\}\right\}\{\{T, U\}\} /\left(h(T), U h^{\prime}(T)-1\right),
$$

where $h$ is a monic polynomial over $\mathscr{A}\left\{\left\{T_{1}, \ldots, T_{d}\right\}\right\}$.

Now consider the special case when $\mathfrak{Y}$ is locally topologically finite type over $\operatorname{Spf} C$. We may apply base change by $\operatorname{Spf} R \rightarrow \operatorname{Spf} C$ and use Raynaud's theory of formal models to reduce the remaining assertions to the analogous claims for Raynaud's functor from formal $R$-models to rigid spaces over $k$. These analogous claims are obvious with two exceptions: (d) without discreteness conditions on $\left|k^{\times}\right|$is a theorem of Temkin [Te, Cor. 4.4, Cor. 4.5], and for (f) and (g) we use Theorem A.2.1.

To reduce the general case to the case when $\mathfrak{Y}$ is locally topologically of finite type over $\operatorname{Spf} C$ we may suppose $\mathfrak{Y}=\operatorname{Spf} \mathscr{A}$ is affine, so there is an isomorphism

$$
\mathscr{A} \simeq\left(C \llbracket q_{1}, \ldots, q_{n} \rrbracket\right)\left\{\left\{T_{1}, \ldots, T_{m}\right\}\right\} /\left(h_{1}, \ldots, h_{r}\right)=(C \llbracket q \rrbracket)\{\{\underline{T}\}\} /\left(h_{1}, \ldots, h_{r}\right)
$$

as topological $C$-algebras (where $C \llbracket q \rrbracket$ has the $(I, q)$-adic topology, with $I$ any ideal of definition of $C$ ). We may replace $C$ with its image in $R$. If the restriction 
of the absolute value to $C$ is trivial then the fraction field of $C$ is contained in $R$, and so upon choosing a nonzero $\pi \in \mathfrak{m}_{R}$ we get a subring $C \llbracket \pi \rrbracket \subseteq R$ that we may rename as $C$ to get to the case when the absolute value for $k$ is non-trivial on the subring $C$.

Choose a nonzero $c \in C$ that is topologically nilpotent, so $0<|c|<1$. Let $\mathfrak{f}_{c}: \mathfrak{X}_{c} \rightarrow \mathfrak{Y}_{c}=\operatorname{Spf} \mathscr{A}_{c}$ denote the base change of $\mathfrak{f}$ by the map

$$
\mathscr{A} \rightarrow \mathscr{A}_{c}=C\{\{\underline{q}, \underline{T}\}\} /\left(h_{1}(c \underline{q}, \underline{T}), \ldots, h_{r}(c \underline{q}, \underline{T})\right)
$$

defined by $q_{j} \mapsto c q_{j}, T_{i} \mapsto T_{i}$. Clearly $\mathfrak{Y}_{c}^{\text {rig }} \subseteq \mathfrak{Y}^{\text {rig }}$ is the admissible open where the Zariski-closed locus

$$
\mathfrak{Y}^{\text {rig }} \hookrightarrow \Delta^{n} \times \mathbf{B}^{m}
$$

meets $\left\{\left|q_{1}\right|, \ldots,\left|q_{n}\right| \leq|c|\right\} \times \mathbf{B}^{m}$, and the restriction of $f^{\text {rig }}$ over $\mathfrak{Y}_{c}^{\text {rig }}$ is identified with $\mathfrak{f}_{c}^{\text {rig }}$.

For $0<r<1$ with $r \in \sqrt{ }\left|k^{\times}\right|$, define the admissible open $Y_{r}=\mathfrak{Y}^{\text {rig }} \cap$ $\left\{\left|q_{1}\right|, \ldots,\left|q_{n}\right| \leq r\right\}$, so $\mathfrak{Y}_{c}^{\text {rig }}=Y_{|c|}$. Since $\left\{Y_{r}\right\}_{r}$ is an admissible open covering of $\mathfrak{Y}^{\text {rig }}$, it suffices to verify the desired properties for each restriction $\left.\mathfrak{f}^{\text {rig }}\right|_{Y_{r}}$. For any particular $r$ it suffices to check the properties after finite extension on $k$ (as everything under consideration descends through such extensions), so upon replacing $C$ and $k$ with suitable finite extensions (depending on $r$ ) it suffices to treat the case when $r=|c|$ for some $c \in C$. We are now done, because $\left.\mathfrak{f}^{\text {rig }}\right|_{Y_{|c|}}=\mathfrak{f}_{c}^{\text {rig }}$ and we know the results for $\mathfrak{f}_{c}$ by the settled case when $\mathfrak{Y}$ is topologically of finite type over $\operatorname{Spf} C$.

We are now in position to construct the desired generalized elliptic curves over $\Delta$. Using Theorem 3.1.6 with $C=\mathbf{Z}$, we get a proper flat curve

$$
\underline{\text { Tate }}_{n}^{\text {an }} \stackrel{\text { def }}{=} \widehat{\text { Tate }}_{n}^{\text {rig }}
$$

over the open unit disc $\Delta=\left(\operatorname{Spf} \mathbf{Z} \llbracket q^{1 / n} \rrbracket\right)^{\text {rig }}$, and there is a finite étale map $\underline{\text { Tate }}_{n}^{\text {an }} \rightarrow \underline{\text { Tate }}_{m}^{\text {an }}$ of degree $n / m$ whenever $m \mid n$ (induced by a finite étale map of degree $n / m$ between formal schemes ${\underline{\underline{\text { Tate }}_{n}}}_{n} \rightarrow{\widehat{\text { Tate }_{m}}}_{m} \mathbf{Z} \llbracket q^{1 / n} \rrbracket)$.

For $n>1$, the construction of $\widehat{\text { Tate }}_{n}$ as a formal scheme via gluing formal annuli implies that the rigid space Tate $_{n}^{\text {an }}$ is covered by $n$ relative annuli $\Delta_{i}^{\text {rig }}=\left\{\left(x_{i}, y_{i}, q\right)|| x_{i}|,| y_{i}|\leq 1| q \mid,<1, x_{i} y_{i}=q\right\}$ that are glued exactly as in the construction of $\widehat{\text { Tate }}_{n}$ for $n>1$.

For $n \geq 1$ it follows from the structure of the formal scheme $\widehat{\text { Tate }}_{n}$ that $\underline{\text { Tate }}_{n}^{\text {an }}$ has $n$-gon fiber over 0 , and by identifying a point $q_{0} \in \Delta(k)-\{0\}$ with the morphism $\operatorname{Sp}(k) \rightarrow \Delta$ induced by the map $\mathbf{Z} \llbracket q^{1 / n} \rrbracket \rightarrow R$ satisfying $q^{1 / n} \mapsto q_{0}$, the fiber $\underline{\text { Tate }}_{n}^{\text {an }}$ over $q_{0}$ is

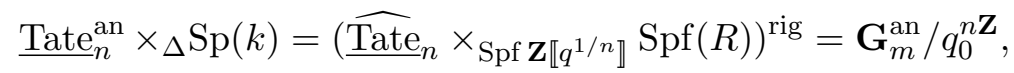


where the final identification uses the construction of the formal scheme $\widehat{\text { Tate }}_{n}$ in terms of gluing formal annuli (and for $n=1$ we must also use the finite étale degree- $m$ covering $\widehat{\text { Tate }}_{m}(q) \rightarrow \widehat{\text { Tate }}_{1}$ over $\mathbf{Z} \llbracket q \rrbracket$ for any choice of $m>1$ ). Since Tate $_{n}^{\text {an }} \rightarrow \Delta$ is a proper flat curve and we have calculated that its fiber over the origin is a standard $n$-gon whereas its fibers away from the origin are smooth and geometrically connected with genus 1 (by the Zariski-local constancy of Euler characteristics in rigid-analytic proper flat families), we conclude that $\underline{T a t e}_{n}^{\text {an }}$ is a DR semistable genus-1 marked curve over $\Delta$.

The relatively ample relative effective Cartier divisor $\mathbf{Z} / n \mathbf{Z} \hookrightarrow{\widehat{\text { Tate }_{n}}}_{n}$ over $\operatorname{Spf}\left(\mathbf{Z} \llbracket q^{1 / n} \rrbracket\right)$ is physically supported in the smooth locus modulo $q^{1 / n}=0$ and is endowed with a canonical action (3.1.1) on the total formal curve. Thus, Theorem 2.2.1 and Lemma B.2.7 (applied to $\operatorname{Pic}_{\underline{\text { Tate }}_{n}\left(q_{0}\right) / R^{\prime}}^{0} \rightarrow \operatorname{Spec} R^{\prime}$ for finite $k^{\prime} / k$ and $\left.q_{0} \in \mathfrak{m}_{R^{\prime}}-\{0\}\right)$ provide a unique structure of generalized elliptic curve on $\underline{\text { Tate }}_{n}^{\text {an }} \rightarrow \Delta$ that extends the unique structure of elliptic curve on Tate $\left.{ }_{n}^{\text {an }}\right|_{\Delta-\{0\}}$ (using the identity section induced from the identity section of $\widehat{\text { Tate }}_{n}$ ).

3.2. Degenerate fibers and modular curves. We now formulate a structure theorem (to be proved later) concerning generalized elliptic curves in rigidanalytic neighborhoods of the locus of degenerate fibers:

Theorem 3.2.1. Let $d \geq 1$ be a fixed integer, and let $f: E \rightarrow S$ be a generalized elliptic curve over a rigid space over a non-archimedean field $k$. In a quasifinite fpqc neighborhood of the locus of d-gon geometric fibers it is isomorphic to a pullback of $\underline{\text { Tate }}_{d}^{\text {an }} \rightarrow \Delta$. More precisely, if $S_{d}^{\infty, f} \subseteq S$ denotes the Zariskiclosed locus of d-gon geometric fibers of $E$ over $S$ then there exists an admissible covering $\left\{S_{i}\right\}$ of an admissible open $U \subseteq S$ containing $S_{d}^{\infty, f}$ such that for suitable quasi-finite fpqc maps $S_{i}^{\prime} \rightarrow S_{i}$ and morphisms $S_{i}^{\prime} \rightarrow \Delta$ there are isomorphisms $E_{/ S_{i}^{\prime}} \simeq$ Tate $_{d}^{\mathrm{an}} \times_{\Delta} S_{i}^{\prime}$ as generalized elliptic curves over $S_{i}^{\prime}$ for all $i$.

If $d \in k^{\times}$then the quasi-finite fpqc maps $S_{i}^{\prime} \rightarrow S_{i}$ can be chosen to be étale.

The idea of the proof is simple: we will verify it near the degenerate fibers on suitable modular curves, and in general we will construct a quasi-finite fpqc-cover around $S_{d}^{\infty, f}$ over which $E \rightarrow S$ acquires enough level structure to be a pullback of the universal curve over a modular curve (and the covering will be able to be chosen to be étale when $d \in k^{\times}$). The proof of Theorem 3.2.1 is given after we prove Theorem 3.2.8 below, and it requires a lot of preparations because we largely use arguments that are applicable in higher-dimensional cases; in [C4] we will use some of the subsequent results and methods.

Let us first introduce the modular curves to be considered. For $\Gamma=\Gamma(N)$ or $\Gamma_{1}(N)$, the notion of a Drinfeld $\Gamma$-structure on a generalized elliptic curve $E_{/ S}$ over a rigid space $S$ is defined exactly as in the algebraic case. (In particular, 
the inverse ideal sheaf of the relative effective Cartier divisor defined by the level structure is required to be relatively ample over the base, in the sense of [C3, Def. 3.2.2].) These notions are compatible with analytification and with change of the base field. There are many other level structures that we could consider, but for simplicity of exposition and sufficiency for our purposes we shall restrict attention to $\Gamma(N)$ and $\Gamma_{1}(N)$. We shall need to consider possibly nonétale Drinfeld structures over schemes in order to treat rigid-analytic generalized elliptic curves whose non-smooth geometric fibers are $d$-gons with $d$ a multiple of the residue characteristic of $k$.

It is a lengthy task to merge the work of Deligne-Rapoport on degenerations and the work of Katz-Mazur on Drinfeld structures on smooth elliptic curves to show that for any $\Gamma$ as above, the moduli stack of Drinfeld $\Gamma$-structures on generalized elliptic curves over variable base schemes is a proper (regular) DeligneMumford stack $\mathscr{M}_{\Gamma}$ over $\mathbf{Z}$. If $S_{0}$ is a scheme and $N$ has a factor $d$ that is a unit on $S_{0}$ such that $d \geq 5$ (resp. $d \geq 3$ ) and $\Gamma=\Gamma_{1}(N)(\operatorname{resp} . \quad \Gamma=\Gamma(N))$, then $\mathscr{M}_{\Gamma / S_{0}}$ is a scheme. We have to use these "well-known" results concerning moduli schemes in what follows, so we refer to [C2, Thm. 3.2.7, Thm. 4.2.1(2)] for proofs.

Lemma 3.2.2. Let $(E, \iota)$ be a generalized elliptic curve with $\Gamma$-structure over a rigid space $S$, where $\Gamma=\Gamma(N)$ or $\Gamma_{1}(N)$ for some $N \geq 1$. There is an admissible open covering $\left\{S_{i}\right\}$ of $S$ such that $(E, \iota)_{/ S_{i}}$ is the pullback of a generalized elliptic curve with $\Gamma$-structure over a locally finite type $k$-scheme.

Proof. By Corollary 2.2.5 we may assume that there is a generalized elliptic curve $\mathscr{E} / \mathscr{S}$ over a locally finite type $k$-scheme $\mathscr{S}$ and a $k$-morphism $S \rightarrow \mathscr{S}$ that pulls $\mathscr{E} / \mathscr{S}$ back to $E_{/ S}$. Using the methods in $[\mathrm{KM}, \mathrm{Ch} .1]$, many of which are applicable to the smooth loci on generalized elliptic curves (viewed as separated group curves), the functor of $\Gamma$-structures on $\mathscr{E}$ is represented by a scheme $\mathscr{S}_{\Gamma}$ that is of finite type over $\mathscr{S}$ (it suffices to work locally on $\mathscr{S}$, and so by Lemma B.2.2 we may suppose that the degenerate fibers have a fixed number of geometric irreducible components). Using the compatibility of algebraic and analytic Hom functors [C3, Cor. 4.1.5], as well as considerations with infinitesimal fibers, $\mathscr{S}_{\Gamma}^{\text {an }} \rightarrow \mathscr{S}^{\text {an }}$ has the analogous universal property for $\mathscr{E}^{\text {an }} \rightarrow \mathscr{S}^{\text {an }}$ in the rigidanalytic category. Thus, the $\Gamma$-structure $\iota$ on $E_{/ S}$ gives rises to a unique morphism of rigid spaces $S \rightarrow \mathscr{S}_{\Gamma}^{\text {an }}$ that pulls the universal $\Gamma$-structure on $\mathscr{E}^{\text {an }} \times \mathscr{S}_{\text {an }} \mathscr{S}_{\Gamma}^{\text {an }}$ back to $\iota$. Composing with $\mathscr{S}_{\Gamma}^{\text {an }} \rightarrow \mathscr{S}_{\Gamma}$ shows that $\left(E_{/ S}, \iota\right)$ is the pullback of the universal $\Gamma$-structure on $\mathscr{E}_{/} \mathscr{S}_{\Gamma}$.

The universality of analytified algebraic modular curves is given by: 
Theorem 3.2.3. Let $\mathscr{M}=\mathscr{M}_{\Gamma / k}$ for $\Gamma=\Gamma_{1}(N)$ or $\Gamma=\Gamma(N)$. Assume that $N$ has a factor $d$ not divisible by $\operatorname{char}(k)$ such that $d \geq 5$ or $d \geq 3$ respectively, so $\mathscr{M}$ is a proper $k$-scheme.

The analytification $\mathscr{M}^{\text {an }}$ of $\mathscr{M}$, equipped with its analytified universal object $\left(\mathscr{E}^{\mathrm{an}}, \iota^{\text {an }}\right)$, is a universal object in the fibered category of generalized elliptic curves with $\Gamma$-structure over rigid spaces over $k$. In particular, the formation of this universal object is compatible with arbitrary analytic change in the non-archimedean base field.

Proof. Note that $\Gamma$-structures over rigid spaces over $k$ admit no non-trivial automorphisms, due to the hypotheses on $N$. Let $(E, \iota)$ be a $\Gamma$-structure over a rigid space $S$. We seek a unique map $S \rightarrow \mathscr{M}^{\text {an }}$ such that the pullback of $\left(\mathscr{E}^{\mathrm{an}}, \iota^{\text {an }}\right)$ is (necessarily uniquely) isomorphic to $(E, \iota)$. By Lemma 2.1.12, uniqueness (but not existence) is reduced to the case of artin local $S=\operatorname{Sp}\left(A_{0}\right)$, where it follows from GAGA over artin rings and the identification of rigid analytic maps $S \rightarrow \mathscr{M}^{\text {an }}$ with $k$-scheme maps $\operatorname{Spec}\left(A_{0}\right) \rightarrow \mathscr{M}$ (since $A_{0}$ is artin local, $\left.\operatorname{Spec}\left(A_{0}\right)^{\text {an }}=\operatorname{Sp}\left(A_{0}\right)\right)$. With uniqueness proved, we may work locally for existence. Local existence follows from Lemma 3.2.2 and universality of the $\Gamma$-structure over $\mathscr{M}_{\Gamma / k}$ in the category of $k$-schemes.

The first step in the proof of Theorem 3.2.1 is an application of Berthelot's functor (as in Theorem 3.1.5) to construct a class of (usually non-quasi-compact) admissible open covers that are well-behaved with respect to arbitrary analytic extension of the non-archimedean base field. For applications to abelian varieties in [C4], and to clarify the underlying structure that really matters in proofs, the subsequent discussion is given in much more generality than we require for the proof of Theorem 3.2.1.

We begin with some notation. Let $R_{0}$ be a complete discrete valuation ring with fraction field $k_{0}, X_{0}$ a separated $R_{0^{-}}$scheme of finite type, and $Y_{0}$ an $R_{0^{-}}$ proper closed subscheme in $X_{0}$. Let $\left\{Z_{\alpha}\right\}$ be a finite set of locally closed subsets of $X_{0}$ whose union contains $Y_{0}$.

Example 3.2.4. The example that we have in mind is to take $X_{0}$ to be an open neighborhood of the boundary $Y_{0}$ on a proper moduli space, and the $Z_{\alpha}$ 's to be locally closed sets in a Zariski-stratification of $Y_{0}$.

Let $\widehat{X}_{\alpha}$ be the formal completion of $X_{0}$ along $Z_{\alpha}$. For all $\alpha$ we consider the formal completion $\widehat{X}_{\alpha}$ as a formal scheme over $\operatorname{Spf}\left(R_{0}^{\text {disc }}\right)=\operatorname{Spec}\left(R_{0}\right)$, where $R_{0}^{\text {disc }}$ is the ring $R_{0}$ considered with the discrete topology. The continuous map $R_{0}^{\text {disc }} \rightarrow R_{0}$ gives a natural $k_{0}$-map

$$
\widehat{X}_{\alpha}^{\mathrm{rig}} \rightarrow X_{0 / k_{0}}
$$


to the generic fiber of the $R_{0}$-scheme $X_{0}$ (induced by the maps $\operatorname{Spf} \widehat{A} \rightarrow \operatorname{Spec} A$ for open affines Spec $A$ in $X_{0}$ that meet the locally closed $Z_{\alpha}$ in a closed subset of $X_{0}$, with $\widehat{A}$ denoting the formal completion of $A$ along the closed subset $(\operatorname{Spec} A) \cap Z_{\alpha}$ ). The universal property of analytification provides a unique map of rigid spaces over $k_{0}$

$$
\widehat{X}_{\alpha}^{\mathrm{rig}} \rightarrow X_{0 / k_{0}}^{\mathrm{an}}
$$

that is compatible with (3.2.1).

Example 3.2.5. If $X_{0}=\mathbf{A}_{R_{0}}^{n}$ and $Z_{\alpha}=Y_{0}$ is the zero-section then (3.2.2) is the canonical map from the closed unit $n$-ball into rigid-analytic affine $n$-space, and $Y_{0 / k_{0}}^{\mathrm{an}}$ is the common origin in each.

Theorem 3.2.6. With notation and hypotheses as above, the natural maps in (3.2.2) are open immersions. The union $U_{0}=\cup_{\alpha} \widehat{X}_{\alpha}^{\mathrm{rig}}$ in $X_{0 / k_{0}}^{\mathrm{an}}$ is an admissible open that contains the analytic set $Y_{0 / k_{0}}^{\mathrm{an}}$, and the $\widehat{X}_{\alpha}^{\mathrm{rig}}$ 's are an admissible cover of $U_{0}$.

For any analytic extension field $k / k_{0}$, the maps

$$
k \widehat{\otimes}_{k_{0}} \widehat{X}_{\alpha}^{\mathrm{rig}} \rightarrow k \widehat{\otimes}_{k_{0}} X_{0 / k_{0}}^{\mathrm{an}} \simeq X_{0 / k}^{\mathrm{an}}, \quad k \widehat{\otimes}_{k_{0}} U_{0} \rightarrow k \widehat{\otimes}_{k_{0}} X_{0 / k_{0}}^{\mathrm{an}} \simeq X_{0 / k}^{\mathrm{an}}
$$

are open immersions with $k \widehat{\otimes}_{k_{0}} U_{0}$ containing $Y_{0 / k}^{\text {an }}$, and the admissible opens $k \widehat{\otimes}_{k_{0}} \widehat{X}_{\alpha}^{\mathrm{rig}}$ are an admissible cover of $k \widehat{\otimes}_{k_{0}} U_{0}$.

This is a mild strengthening of [deJ, 7.2.5], allowing extension of scalars $k / k_{0}$ to a non-archimedean field $k$ that is not assumed to be discretely-valued; we need to give a proof for the preservation of admissibility conditions with respect to such an extension of scalars because it is not known (and seems doubtful) in general that the functor $k \widehat{\otimes}_{k_{0}}(\cdot)$ carries open immersions to open immersions and admissible covers to admissible covers when we allow open immersions that are not assumed to be quasi-compact. The case of open immersions that are not quasi-compact arises in Example 3.2.5 and in $\S 4$ (as well as in the analogous higher-dimensional setting in [C4]).

Proof. We proceed in several steps to ultimately reduce to a calculation with affinoid rigid spaces.

Step 1. We first reduce ourselves to a problem for formal schemes over $\operatorname{Spf}\left(R_{0}\right)$. Let $\widehat{X}_{\alpha}^{\prime}$ denote the formal completion of $X_{0}$ along the closed fiber of $Z_{\alpha}$ over $\operatorname{Spec}\left(R_{0}\right)$, so

$$
\widehat{X}_{\alpha}^{\prime} \simeq \operatorname{Spf}\left(R_{0}\right) \times_{\operatorname{Spf}\left(R_{0}^{\text {disc }}\right)} \widehat{X}_{\alpha}
$$

where $R_{0}^{\text {disc }}$ denotes the ring $R_{0}$ with the discrete topology. By the compatibility with "change in $C$ " in Theorem 3.1.5, applied to $R_{0}^{\text {disc }} \rightarrow R_{0}$, it follows from 
(3.2.3) that each $\widehat{X}_{\alpha}^{\text {rig }}$ is naturally identified with a "Berthelot analytification" $\left(\widehat{X}_{\alpha}^{\prime}\right)^{\text {rig }}$ on the category $\mathrm{FS}_{R_{0}}$. We conclude that if $\mathfrak{X}_{0}$ denotes the formal completion of $X_{0}$ along its closed fiber then (3.2.2) naturally factors through the canonical map

$$
i_{X_{0}}: \mathfrak{X}_{0}^{\mathrm{rig}} \rightarrow X_{0 / k_{0}}^{\mathrm{an}}
$$

Since Berthelot's functor coincides with Raynaud's functor on the category of formal schemes that are topologically of finite type over $R_{0}$, the $R_{0}$-separatedness of $X_{0}$ implies that the map $i_{X_{0}}$ is a quasi-compact open immersion [C1, 5.3.1]. The formal completion $\mathfrak{X}_{0}$ of $X_{0}$ makes sense even when $R_{0}$ is just a complete (rank 1) valuation ring, by using formal completion with respect to an ideal of definition of the valuation ring (rather than the maximal ideal). In this sense, the formation of $i_{X_{0}}$ as a quasi-compact open immersion is compatible with arbitrary extension on the analytic ground field $([\mathrm{C} 1,5.3 .1]$ is given over a general nonarchimedean base field). Thus, we are reduced to studying the maps $\widehat{X}_{\alpha}^{\prime} \rightarrow \mathfrak{X}_{0}$ of formal schemes over $\operatorname{Spf}\left(R_{0}\right)$. We have to prove three things:

(1) Working with Berthelot's functor on the category $\mathrm{FS}_{R_{0}}$, the induced maps $\left(\widehat{X}_{\alpha}^{\prime}\right)^{\text {rig }} \rightarrow \mathfrak{X}_{0}^{\text {rig }}$ are open immersions and remain open immersions after any analytic extension on the base field.

(2) The finitely many admissible opens $\left(\widehat{X}_{\alpha}^{\prime}\right)^{\text {rig }}$ in $\mathfrak{X}_{0}^{\text {rig }}$ are an admissible covering of their union $U_{0}$, and both this property and the formation of $U_{0}$ are compatible with any analytic extension on the base field.

(3) The locus $U_{0}$ in $X_{0 / k_{0}}^{\text {an }}$ contains $Y_{0 / k_{0}}^{\mathrm{an}}$.

Step 2. Let us address (3). Since (1) has not yet been proved, we will consider the alternative (but equivalent, under (1)) assertion that the union of the images of the $\left(\widehat{X}_{\alpha}^{\prime}\right)^{\text {rig }}$ 's in $\mathfrak{X}_{0}^{\text {rig }} \subseteq X_{0 / k_{0}}^{\text {an }}$ contains $Y_{0 / k_{0}}^{\text {an }}$. Let $\mathfrak{Y}_{0}$ denote the completion of $Y_{0}$ along its closed fiber. Since $Y_{0}$ is $R_{0}$-proper, [C1, 5.3.1] implies that the natural map $i_{Y_{0}}: \mathfrak{Y}_{0}^{\text {rig }} \rightarrow Y_{0 / k_{0}}^{\text {an }}$ is an isomorphism. By Lemma A.1.5, for any $y \in Y_{0 / k_{0}}^{\text {an }}$ there exists a finite flat (local) domain $R_{0}^{\prime}$ over $R_{0}$ and a map $\operatorname{Spf}\left(R_{0}^{\prime}\right) \rightarrow \mathfrak{Y}_{0}$ that induces $y$ upon applying Berthelot's functor (since this functor coincides with Raynaud's functor on the category of formal schemes that are topologically of finite type over $R_{0}$ ). The composite map

$$
\operatorname{Spf}\left(R_{0}^{\prime}\right) \rightarrow \mathfrak{Y}_{0} \rightarrow \mathfrak{X}_{0}
$$

has its closed point landing in the closed fiber of one of the $Z_{\alpha}$ 's because the $Z_{\alpha}$ 's cover $Y_{0}$ set-theoretically. Thus, the topology of $\operatorname{Spf}\left(R_{0}^{\prime}\right)$ implies that the map (3.2.4) of formal schemes over $\operatorname{Spf}\left(R_{0}\right)$ factors through the formal completion $\widehat{X}_{\alpha}^{\prime}$ of $X_{0}$ along the closed fiber of some $Z_{\alpha}$. Applying Berthelot's functor to this factorization in $\mathrm{FS}_{R_{0}}$, we conclude that $y \in Y_{0 / k_{0}}^{\text {an }}$ considered as a point of 
$\mathfrak{X}_{0}^{\text {rig }} \subseteq X_{0 / k_{0}}^{\text {an }}$ lies in the image of $\left(\widehat{X}_{\alpha}^{\prime}\right)^{\text {rig }}$ for some $\alpha$. This concludes our treatment of (3).

Step 3. For assertions (1) and (2), we can rephrase our problems as general problems concerning formal schemes (that is, we can eliminate the intervention of ordinary schemes over $R_{0}$ or $k_{0}$ ), as follows. Let $\mathfrak{X}$ be an arbitrary object in the category $\mathrm{FS}_{R_{0}}$. Let $\left\{Z_{\alpha}\right\}$ be a finite set of locally closed subschemes in the ordinary scheme $\mathfrak{X}_{\text {red }}$ over the residue field $R_{0} / \mathfrak{m}_{R_{0}}$, and let $\mathfrak{Z}_{\alpha}$ denote the formal completion of $\mathfrak{X}$ along $Z_{\alpha}$. There are canonical maps $\mathfrak{Z}_{\alpha} \rightarrow \mathfrak{X}$, and we wish to prove two assertions concerning rigid spaces:

(1) The induced maps $\mathfrak{Z}_{\alpha}^{\text {rig }} \rightarrow \mathfrak{X}^{\text {rig }}$ on Berthelot analytifications are open immersions and remain so after arbitrary analytic extension on the base field.

(2) The union $U$ of the admissible opens $\mathfrak{Z}_{\alpha}^{\text {rig }}$ in $\mathfrak{X}^{\text {rig }}$ is an admissible open such

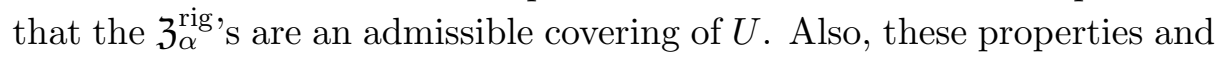
the formation of $U$ are all compatible with arbitrary analytic extension of the base field.

These problems are local on $\mathfrak{X}$, so we may suppose that all $Z_{\alpha}$ 's are closed in $\mathfrak{X}_{\text {red }}$. Thus, by [deJ, 7.2.5], each map

$$
\mathfrak{Z}_{\alpha}^{\text {rig }} \rightarrow \mathfrak{X}^{\text {rig }}
$$

is an open immersion. To keep track of admissibility and the effect of change in the ground field we may work locally on $\mathfrak{X}$, and so we may suppose that $\mathfrak{X}=\operatorname{Spf}(\mathscr{A})$ is affine.

Step 4. Let $f_{1}^{(\alpha)}, \ldots, f_{r_{\alpha}}^{(\alpha)} \in \mathscr{A}$ be representatives for generators of the ideal of $Z_{\alpha}$ in the quotient $\mathscr{A}_{\text {red }}$ of $\mathscr{A}$ by topological nilpotents. We have $\mathfrak{Z}_{\alpha}=\operatorname{Spf}\left(\mathscr{B}_{\alpha}\right)$ where $\mathscr{B}_{\alpha}$ is the $\left(f_{1}^{(\alpha)}, \ldots, f_{r_{\alpha}}^{(\alpha)}\right)$-adic completion of $\mathscr{A}$, or equivalently $\mathscr{B}_{\alpha} \simeq$ $\mathscr{A} \llbracket T_{1}, \ldots, T_{r_{\alpha}} \rrbracket /\left(T_{j}-f_{j}^{(\alpha)}\right)$ as topological $\mathscr{A}$-algebras. By the functoriality of $(\cdot)^{\text {rig }}$, it follows that $\mathfrak{Z}_{\alpha}^{\text {rig }}$ is identified with the locus in $\mathfrak{X}^{\text {rig }} \times \Delta^{r_{\alpha}}$ cut out by the conditions $T_{1}=f_{1}^{(\alpha)}, \cdots, T_{r_{\alpha}}=f_{r_{\alpha}}^{(\alpha)}$, and under this identification the projection $\mathfrak{X}^{\text {rig }} \times \Delta^{r_{\alpha}} \rightarrow \mathfrak{X}^{\text {rig }}$ induces (3.2.5). Thus, we can reformulate our problem entirely in terms of rigid spaces, without the intervention of formal schemes, as follows.

Let $X$ be an arbitrary rigid space over a non-archimedean field $k$, and let $f_{j}^{(\alpha)} \in \mathrm{H}^{0}\left(X, \mathscr{O}_{X}\right)$ be finitely many elements, with $1 \leq j \leq r_{\alpha}$ for $\alpha$ ranging through a finite set. Define $Z_{\alpha} \hookrightarrow X \times \Delta^{r_{\alpha}}$ to be the zero locus of the finitely many differences $T_{j}-f_{j}^{(\alpha)}$ for $1 \leq j \leq r_{\alpha}$ (with $T_{1}, \ldots, T_{r_{\alpha}}$ the coordinates on $\left.\Delta^{r_{\alpha}}\right)$. We make two claims: 
(1) The projection $X \times \Delta^{r_{\alpha}} \rightarrow X$ induces an isomorphism of $Z_{\alpha}$ onto the admissible open $U_{\alpha}$ in $X$ defined by

$$
\left|f_{1}^{(\alpha)}\right|<1, \cdots,\left|f_{r_{\alpha}}^{(\alpha)}\right|<1
$$

(the formation of the locus (3.2.6) clearly commutes with any analytic extension on the base field).

(2) The finite union $U=\cup_{\alpha} U_{\alpha}$ is an admissible open in $X$ such that the $U_{\alpha}$ 's are an admissible covering, the formation of the union $U$ commutes with analytic extension on the base field, and the admissibility property for the $U_{\alpha}$ 's as a covering of $U$ is preserved by any analytic extension on the base field.

Since $X \times \Delta^{r_{\alpha}}$ is the locus in $X \times \mathbf{B}^{r_{\alpha}}$ cut out by the conditions $\left|T_{1}\right|, \ldots,\left|T_{r_{\alpha}}\right|<$ 1 , it suffices to work with the map $X \times \mathbf{B}^{r_{\alpha}} \rightarrow X$ and the conditions $\left|f_{j}^{(\alpha)}\right| \leq 1$ for all $1 \leq j \leq r_{\alpha}$ and all $\alpha$. We may work locally on $X$, and so we can assume that $X$ is affinoid. In this case all $U_{\alpha}$ are affinoid, and everything is therefore obvious.

Corollary 3.2.7. Let $R_{0}$ be a complete discrete valuation ring with fraction field $k_{0}$, and let $X$ be a separated $R_{0}$-scheme of finite type. Let $f: P \rightarrow X$ be a proper morphism, and let $Z \hookrightarrow X$ be a locally closed subset. Let $\widehat{X}$ and $\widehat{P}$ denote the formal completions of $X$ and $P$ along $Z$ and $f^{-1}(Z)$ respectively. The commutative square of rigid spaces

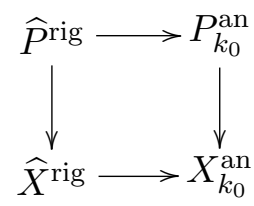

is cartesian.

By Theorem 3.2.6 (with $Y_{0}$ taken to be empty), the horizontal maps in (3.2.7) are open immersions. One example we have in mind is for $P \rightarrow X$ to be a proper universal object over an open locus in a moduli space.

Proof. By [Ber1, 3.4.7] and [Te, Cor. 4.5], analytification carries proper algebraic maps to proper rigid-analytic maps. Hence, the right side of (3.2.7) is proper. Likewise, since $\widehat{P} \rightarrow \widehat{X}$ is proper, it follows from Theorem 3.1.6 that the left side of (3.2.7) is proper. Thus, the induced map

$$
\widehat{P}^{\text {rig }} \rightarrow \widehat{X}^{\text {rig }} \times_{X_{k_{0}}^{\text {an }}} P_{k_{0}}^{\text {an }}
$$

over $\widehat{X}^{\text {rig }}$ is a quasi-compact open immersion, so it is an isomorphism if and only if it is bijective. 
By (3.2.3) applied to both $X$ and $P$, we can replace the formal schemes $\widehat{X}$ and $\widehat{P}$ over $\operatorname{Spf}\left(R_{0}^{\text {disc }}\right)$ in (3.2.8) with the formal completions $\widehat{X}^{\prime}$ and $\widehat{P}^{\prime}$ of $X$ and $P$ along the closed fiber of $Z$ (and its preimage in $P$ ) over Spec $R_{0}$. The formal schemes $\widehat{X}^{\prime}$ and $\widehat{P}^{\prime}$ over $\operatorname{Spf}\left(R_{0}\right)$ lie in $\mathrm{FS}_{R_{0}}$. It is obvious from the construction of $\left(\widehat{X}^{\prime}\right)^{\text {rig }}$ that for every point $\xi$ of $\left(\widehat{X}^{\prime}\right)^{\text {rig }}$ there exists a discrete valuation ring $R_{0}^{\prime}$ finite flat over $R_{0}$ and an $R_{0}$-morphism $x: \operatorname{Spec}\left(R_{0}^{\prime}\right) \rightarrow X$ sending the closed point into $Z$ such that $\xi=\widehat{x}^{\text {rig }}$ where $\widehat{x}: \operatorname{Spf}\left(R_{0}^{\prime}\right) \rightarrow\left(\widehat{X}^{\prime}\right)^{\text {rig }}$ is the induced map in $\mathrm{FS}_{R_{0}}$ between formal completions along the closed point and along $Z$ respectively. Since our remaining problem is set-theoretic, and $(\cdot)^{\text {rig }}$ and $(\cdot)^{\text {an }}$ are compatible with fiber products, by using pullback along $\widehat{x}^{\text {rig }}$ and $x_{/ k_{0}}^{\text {an }}$ we may reduce to the case $X=\operatorname{Spec} R_{0}^{\prime}$. We can rename $R_{0}^{\prime}$ as $R_{0}$ to reduce to the case when $X=\operatorname{Spec} R_{0}$ and $Z$ is its closed point (the other option, $Z=X$, is trivial). In this case $P$ is a proper scheme over Spec $R_{0}$ and the cartesian property of (3.2.7) in this case is the assertion that the canonical (quasi-compact) open immersion $i_{P}: \mathfrak{P}^{\text {rig }} \rightarrow P_{k_{0}}^{\text {an }}$ is bijective (or equivalently, an isomorphism), where $\mathfrak{P}$ is the formal completion of $P$ along its closed fiber over Spec $R_{0}$. The fact that $i_{P}$ is bijective is a consequence of the valuative criterion for properness (or see [C1, $5.3 .1(4)])$.

Now we turn to proving Theorem 3.2.1 over certain modular curves, and this case will then be used to settle the general case. The only input that we require from the theory of modular curves is a good theory of proper moduli spaces over the valuation ring (analogous input in the higher-dimensional case is provided by work of Chai and Faltings [CF, IV 6.7; V, 5.8]); the 1-dimensionality of modular curves is not an essential ingredient in the method that we use.

Theorem 3.2.8. Let $N$ be a positive integer divisible by an integer $n \geq 5$ with $n \in R^{\times}$, and let $\mathbf{Z}^{\prime}$ denote the localization of $\mathbf{Z}$ at the prime ideal $\operatorname{ker}\left(\mathbf{Z} \rightarrow R / \mathfrak{m}_{R}\right)$, so $\mathscr{M}=\mathscr{M}_{\Gamma_{1}(N) / \mathbf{Z}^{\prime}}$ is a scheme over $\mathbf{Z}^{\prime}$. For any $M \geq 1$, let $k_{M}=k \otimes \mathbf{Z} \mathbf{Z}\left[\zeta_{M}\right]$ and let $k_{M}^{+}=k \otimes \mathbf{Z} \mathbf{Z}\left[\zeta_{M}\right]^{+}$, where $\mathbf{Z}\left[\zeta_{M}\right]$ and $\mathbf{Z}\left[\zeta_{M}\right]^{+}$denote the $M$ th cyclotomic integer ring and its maximal totally real subring respectively.

There exists a finite set of $\Gamma_{1}(N)$-structures on generalized elliptic curves over open discs of the form $k_{N / d} \otimes_{k} \Delta$ for various $d \mid N$ with $d>2$ and over open discs of the form $k_{N / d}^{+} \otimes_{k} \Delta$ for various $d \mid N$ with $d \leq 2$ such that the induced maps from these open discs to $\mathscr{M}_{/ k}^{\text {an }}$ are open immersions whose images constitute an admissible open covering of the locus of degenerate fibers.

For each $d \mid N$ with $d>2$ (resp. $d \leq 2$ ) the generalized elliptic curves for the $\Gamma_{1}(N)$-structures over $k_{N / d} \otimes_{k} \Delta$ (resp. $\left.k_{N / d}^{+} \otimes_{k} \Delta\right)$ become isomorphic to the pullback of the Tate family Tate $_{d}^{\text {an }}$ over $k_{N} \otimes_{k} \Delta$ via multiplication on the standard coordinate of $\Delta$ by a suitable power of $\zeta_{N}^{d} \in k_{N}^{\times}$. 
Remark 3.2.9. The method of proof of Theorem 3.2.8 applies to all standard Drinfeld level structures that admit no non-trivial automorphisms when working with $\mathbf{Z}^{\prime}$-schemes. The implicit roots of unity that intervene in the twisted Tate families at the end of Theorem 3.2.8 are made explicit in [C2, Thm. 4.3.6, Thm. 4.3.7].

Proof. Let $\mathscr{M}^{\infty} \hookrightarrow \mathscr{M}$ be the locus of non-smoothness for the universal generalized elliptic curve over $\mathscr{M}$, so $\mathscr{M}^{\infty}$ is proper (even finite) over $\mathbf{Z}^{\prime}$. It follows from the integral theory of the formal structure along the cusps that $\mathscr{M}^{\infty}$ is a union of finitely many closed subsets $\mathscr{M}_{i}^{\infty}$ such that the formal completion of $\mathscr{M}$ along each $\mathscr{M}_{i}^{\infty}$ is isomorphic to a formal spectrum with one of two forms: $\operatorname{Spf}\left(\mathbf{Z}^{\prime}\left[\zeta_{N / d}\right] \llbracket t \rrbracket\right)$ for some $d \mid N$ with $d>2$, or $\operatorname{Spf}\left(\mathbf{Z}^{\prime}\left[\zeta_{N / d}\right]^{+} \llbracket t \rrbracket\right)$ for some $d \mid N$ with $d \leq 2$. Moreover, if we extend scalars to $\mathbf{Z}^{\prime}\left[\zeta_{N}\right]$ then the formal completion of the universal generalized elliptic curve over such a formal spectrum is isomorphic

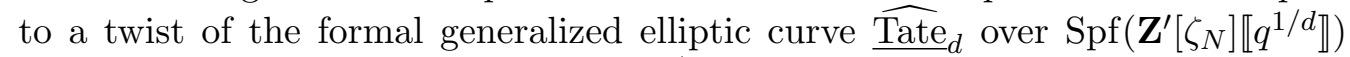
via pullback along multiplication on $q^{1 / d}$ by a suitable power of $\zeta_{N}^{d}$; the details concerning these formal calculations along $\mathscr{M}^{\infty}$ (including the explicit power of $\zeta_{N}^{d}$ that intervenes at each cusp) are given in [C2, §4.3]. We may extend scalars by $\mathbf{Z}^{\prime} \rightarrow R$ to get similar conclusions over $R$. Thus, formally along the cusps on $\mathscr{M}_{/ R}$ the universal generalized elliptic curve becomes isomorphic to a twisted formal Tate curve after passing to a "cyclotomic" covering of $R$. (The only purpose in using $\mathbf{Z}^{\prime}$ rather than $\mathbf{Z}$ above is that the moduli stack over $\mathbf{Z}$ becomes a scheme over $\mathbf{Z}^{\prime}$; when the moduli stack is a scheme over $\mathbf{Z}$ then we can replace $\mathbf{Z}^{\prime}$ with $\mathbf{Z}$ throughout.)

We now bring in Berthelot's functor. By Example 3.1.2, we can find a complete discrete valuation ring $R_{0}$ over which $R$ is faithfully flat. Let $k_{0} \subseteq k$ be the fraction field of $R_{0}$. We shall first work over $R_{0}$ because completion along closed subschemes is better-behaved in the noetherian case. Let us return to the setup for Theorem 3.2.6: $X_{0}$ is a separated $R_{0}$-scheme of finite type, $Y_{0} \hookrightarrow X_{0}$ is an $R_{0}$-proper closed subscheme, and $\left\{Z_{\alpha}\right\}$ is a finite set of locally closed subsets of $X_{0}$ whose union contains $Y_{0}$. (The example we have in mind is in Example 3.2.4 with $X_{0}=\mathscr{M}, Y_{0}=\mathscr{M}^{\infty}$, and $\left\{Z_{\alpha}\right\}$ the set of components $\left\{\mathscr{M}_{i}^{\infty}\right\}$ of $Y_{0}$.) We write $\widehat{X}_{\alpha}$ to denote the formal completion of $X_{0}$ along $Z_{\alpha}$; the use of formal completion along the $Z_{\alpha}$ 's is the reason for initially considering the case of noetherian $R_{0}$ (i.e., the discretely-valued case). We get natural maps of rigid spaces as in (3.2.2).

For the intended application with universal generalized elliptic curves over modular curves, it is important to note that (3.2.2) has a "cartesian" property with respect to proper morphisms. To be precise, if $P_{0} \rightarrow X_{0}$ is a proper morphism and $\widehat{P}_{\alpha}^{\text {rig }}$ denotes the formal completion of $P_{0}$ along the pullback of $Z_{\alpha}$, 
then by Corollary 3.2.7 the commutative square of rigid spaces

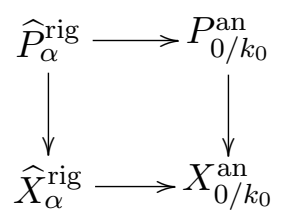

is cartesian. In the context of modular curves we take $\left\{Z_{\alpha}\right\}$ to be the set of components $\left\{\mathscr{M}_{i}^{\infty}\right\}$ of the cuspidal locus; for each $i$ the explicit description of formal completions of modular curves over $\mathbf{Z}^{\prime}$ provides a divisor $d_{i} \mid N$ such that if $d_{i}>2$ there is a cartesian square

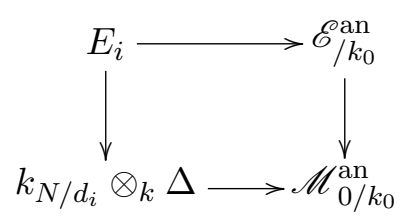

with $k_{N} \otimes_{k_{N / d_{i}}} E_{i}$ over $k_{N} \otimes_{k} \Delta$ isomorphic to the pullback of $k_{N} \otimes_{k} \widehat{\text { Tate }}_{d_{i}}^{\text {rig }}=$ $k_{N} \otimes_{k}$ Tate $_{d_{i}}^{\text {an }}$ by multiplication by a suitable power of $\zeta_{N}^{d}$ on the coordinate of the disc. We likewise obtain similar squares with twisted forms of Tate ${ }_{d_{i}}^{\text {an }}$ using $k_{N / d_{i}}^{+}$instead of $k_{N / d_{i}}$ when $d_{i} \leq 2$. Of course, for a single $d \mid N$ there may be several such squares with $d_{i}=d$ (different maps along the bottom side) as we run through the $\mathscr{M}_{i}^{\infty}$ 's.

It must be proved that the bottom sides of the squares (3.2.9) for $d_{i}>2$ (and the analogues using $k_{N / d_{i}}^{+}$for $d_{i} \leq 2$ ) are open immersions that provide an admissible open cover of an admissible neighborhood of the locus of cusps on $\mathscr{M}_{/ k_{0}}^{\text {an }}$, and it has to be proved that these properties (open immersion, admissibility, and covering) persist after applying the functor $k \widehat{\otimes}_{k_{0}}(\cdot)$.

The bottom side of (3.2.9) is an instance of (3.2.2), so we may apply Theorem 3.2 .6 to (3.2.2) to get the desired results over $k_{0}$. The compatibility with change in the base field in Theorem 3.2.6 gives the desired results for rigid spaces over the initial base field $k$.

Example 3.2.10. The preceding technique of proof (in conjunction with (3.1.4)) provides the justification of the well-known fact (used in $[\mathrm{BT}]$ and $[\mathrm{Bu}]$ ) that a modular form over a number field has the same $q$-expansion whether we work complex-analytically or $p$-adic-analytically.

Finally, we can prove Theorem 3.2.1:

Proof. Let $M \geq 5$ be an integer relatively prime to $d$ such that $M \in k^{\times}$. By working locally on $S$, we may assume via Corollary 2.2.5 that there is a $k$-scheme 
$\mathscr{S}$ locally of finite type and a $k$-map $S \rightarrow \mathscr{S}$ such that $f: E \rightarrow S$ is the pullback of a generalized elliptic curve $f^{\text {alg }}: \mathscr{E} \rightarrow \mathscr{S}$.

Choose any $s \in S^{\infty, f}$, and let $\sigma \in \mathscr{S}^{\infty, f^{\text {alg }}}$ be its closed-point image in $\mathscr{S}$. Let $\bar{\sigma}$ be an algebraic geometric point over $\sigma$ and let $c(\mathscr{E})$ denote the contraction of $\mathscr{E}$ away from its fibral identity components, so over the strictly henselian local ring $\mathscr{O}_{\mathscr{S}, \bar{\sigma}}^{\mathrm{sh}}$ the quasi-finite flat separated group scheme $c(\mathscr{E})^{\mathrm{sm}}[d]$ admits a "finite part": a finite flat closed subgroup scheme that lifts the $d$-torsion on the geometric fiber $c(\mathscr{E}) \frac{\mathrm{sm}}{\sigma}$. This "finite part" is a multiplicative group of order $d$, and so it is isomorphic to $\mu_{d}$ over the strictly henselian base $\mathscr{O}_{\mathscr{S}, \bar{\sigma}}^{\mathrm{sh}}$. Since $c(\mathscr{E})^{\mathrm{sm}}$ is naturally a subgroup of $\mathscr{E}$ sm , we obtain a short exact sequence of finite flat $d$-torsion $\mathscr{O}_{\mathscr{S}, \bar{\sigma}^{-}}^{\mathrm{sh}}$ group schemes $0 \rightarrow \mu_{d} \rightarrow \mathscr{E}^{\operatorname{ssm}}[d] \rightarrow \mathbf{Z} / d \mathbf{Z} \rightarrow 0$, and this sequence must split if $d \in k^{\times}$. If $\operatorname{char}(k) \mid d$, this sequence splits over a finite flat covering of $\operatorname{Spec}\left(\mathscr{O}_{\mathscr{S}, \bar{\sigma}}^{\text {sh }}\right)$. By Lemma B.2.2, the subgroup $\mathbf{Z} / d \mathbf{Z}$ lifted into $\mathscr{E}^{\mathrm{sm}}[d]$ under such a splitting maps isomorphically onto the geometric component groups of the non-smooth fibers over the local base $\operatorname{Spec} \mathscr{O}_{\mathscr{S}, \bar{\sigma}}^{\text {sh }}$ because such an assertion may be checked on the fibers over the unique closed point. The pullback of the quasi-finite, flat, separated group scheme $\mathscr{E}^{\mathscr{s m}}[M]$ over $\mathscr{O}_{\mathscr{S}, \bar{\sigma}}^{\text {sh }}$ also has a "finite part." This "finite part" lifts the $M$-torsion on the fiber $\mathscr{E}_{\bar{\sigma}} \mathrm{sm}$ and hence (due to how we chose $M$ ) it is the constant group $\mathbf{Z} / M \mathbf{Z}$ over our strictly henselian local base.

By using standard limit arguments to descend from a strict henselization (or finite flat covering thereof), we can find a quasi-finite flat separated neighborhood $\mathscr{U}_{\sigma} \rightarrow \mathscr{S}$ of $\sigma$ such that $\left.\mathscr{E}^{\mathrm{sm}}[M]\right|_{\mathscr{U}_{\sigma}}$ contains a closed subgroup scheme $\mathbf{Z} / M \mathbf{Z}$ and $\left.\mathscr{E}^{\operatorname{sm}}[d]\right|_{\mathscr{U}_{\sigma}}$ is isomorphic to $\mathbf{Z} / d \mathbf{Z} \times \mu_{d}$ with $\mathbf{Z} / d \mathbf{Z}$ mapping isomorphically onto the geometric component group of the non-smooth fibers of $\mathscr{E}^{\mathrm{sm}}$ over $\mathscr{U}_{\sigma}$; if $d \in k^{\times}$then we can choose $\mathscr{U}_{\sigma} \rightarrow \mathscr{S}$ to be étale.

Since each map $\mathscr{U}_{\sigma} \rightarrow \mathscr{S}$ has open image, and opens in $\mathscr{S}$ pull back to Zariski-opens of $S$, we may shrink $\mathscr{S}$ and $S$ to reduce to the case that $S$ is quasicompact and $\mathscr{U}_{\sigma} \rightarrow \mathscr{S}$ is surjective for some $\sigma$. We may work locally on $S$, and by the local existence of quasi-compact flat (resp. quasi-compact étale) quasisections to analytifications of faithfully flat (resp. étale surjective) morphisms of algebraic $k$-schemes [C3, Thm. 4.2.2] (whose proof uses Berkovich spaces) we may make a base change by a quasi-finite $f p q c$ morphism (or a quasi-compact étale surjective morphism if $d \in k^{\times}$) to arrange that there exists a closed immersion $\mathbf{Z} / M \mathbf{Z} \hookrightarrow E^{\mathrm{sm}}[M]$ and an isomorphism $E^{\mathrm{sm}}[d] \simeq \mathbf{Z} / d \mathbf{Z} \times \mu_{d}$, where $\mathbf{Z} / d \mathbf{Z}$ maps isomorphically to the geometric component group of all non-smooth fibers over $S$. Letting $N=M d$, the pair of sections $1 \in \mathbf{Z} / M \mathbf{Z}$ and $1 \in \mathbf{Z} / d \mathbf{Z}$ define a $\Gamma_{1}(N)$-structure on $E \rightarrow S$ (this level structure is $S$-ample because of the fibral relationship between $\mathbf{Z} / d \mathbf{Z}$ and the geometric component groups of non-smooth fibers over $S$ ). Thus, by Theorem 3.2.3 there exists a morphism $S \rightarrow \mathscr{M}_{\Gamma_{1}(N) / k}^{\text {an }}$ that pulls the universal generalized elliptic curve back to our given $E_{/ S}$. An 
application of Theorem 3.2.8 now concludes the proof, since $N / d=M$ is not divisible by $\operatorname{char}(k)$ and the $k$-algebra $k_{N}=k \otimes \mathbf{Z} \mathbf{Z}\left[\zeta_{N}\right]$ has a section over the finite étale $k$-algebra $k_{N_{0}}$, with $N_{0}$ the largest factor of $N$ not divisible by $\operatorname{char}(k)$.

\section{Canonical subgroups}

We now focus on the case when $k$ is an analytic extension field of $\mathbf{Q}_{p}$ for some prime $p$, which is to say that the valuation $\operatorname{ring} R$ of $k$ has mixed characteristic $(0, p)$. We impose the normalization condition $|p|=1 / p$. The reader is referred to $\S$ B. 3 for a review of two topics: (i) the algebraic theory of "reduction type" for generalized elliptic curves over $k$ when $k$ is not assumed to be discretely-valued (so the theory of Néron models is not available), and (ii) Lubin's algebraic theory of the $p$-torsion canonical subgroup in generalized elliptic curves over $k$.

4.1. The $p$-torsion case. By Corollary 2.1.14, every rigid-analytic generalized elliptic curve $E_{k}$ over $\operatorname{Sp}(k)$ is the analytification of a unique (up to unique isomorphism) generalized elliptic curve $E_{k}^{\text {alg }}$ over Spec $k$. Thus, the concepts of potentially toric, potentially ordinary, and potentially supersingular reduction may be defined for $E_{k}$ by applying the definitions in the algebraic case to $E_{k}^{\text {alg }}$ (see $\S$ B.3 for the definitions in the algebraic case). Likewise, by using the algebraization we may define the Hasse invariant $h\left(E_{k}\right) \stackrel{\text { def }}{=} h\left(E_{k}^{\text {alg }}\right) \in[1 / p, 1] \cap \sqrt{ }\left|k^{\times}\right|$(see Definition B.3.3); as $h\left(E_{k}\right)$ is made smaller, the potential reduction-type for $E_{k}$ becomes "more supersingular." These definitions on the rigid-analytic side are unaffected by analytic extension on the base field, and $h\left(E_{k}\right)=1$ if and only if $E_{k}$ has potentially toric or potentially ordinary reduction.

Definition B.3.6 and the discussion preceding it give a theory of the canonical subgroup in $E_{k}^{\mathrm{sm}}$ when $h\left(E_{k}\right)>p^{-p /(p+1)}$; the discussion there is given in the algebraic case over $\operatorname{Spec}(k)$, and we carry it over to any rigid-analytic generalized elliptic curve over $\operatorname{Sp}(k)$ by analytifying the canonical subgroup on the algebraization. Our aim below is to give the relativization in rigid-analytic families $E \rightarrow S$. There are two steps: (a) admissibility (and good behavior with respect to change of the base field) for the locus $S^{>p^{-p /(p+1)}}$ of $s \in S$ such that $h\left(E_{s}\right)>p^{-p /(p+1)}$, and (b) construction of (and uniqueness for) the relative canonical subgroup over the locus $S^{>p^{-p /(p+1)}}$ such that it recovers Lubin's theory on fibers and has formation that commutes with base change and with analytic extension of the base field. We begin with (a):

Theorem 4.1.1. Let $E \rightarrow S$ be a generalized elliptic curve over a rigid-analytic space $S$ over $k$. Fix $h \in[1 / p, 1) \cap \sqrt{ }\left|k^{\times}\right|$. Let $S^{>h}$ be the set of $s \in S$ such that $h\left(E_{s}\right)>h$, and define $S^{\geq h}$ similarly for $h \in(1 / p, 1] \cap \sqrt{ }\left|k^{\times}\right|$. 
(1) The loci $S^{>h}$ and $S^{\geq h}$ are admissible opens in $S$ whose formation is compatible with base change on $S$, and the open immersion $S^{\geq h} \rightarrow S$ is quasi-compact. Moreover, for any $h_{0} \in[1 / p, 1) \cap \sqrt{ }\left|k^{\times}\right|$the set of loci $S^{\geq h}$ for all $h \in\left(h_{0}, 1\right) \cap \sqrt{ }\left|k^{\times}\right|$constitutes an admissible cover of $S^{>h_{0}}$.

(2) For quasi-separated or pseudo-separated $S$, the formation of $S^{>h}$ and $S \geq h$ is compatible with change of the base field. In particular, the maps $k^{\prime} \widehat{\otimes}_{k} S^{\geq h} \rightarrow k^{\prime} \widehat{\otimes}_{k} S^{>h} \rightarrow k^{\prime} \widehat{\otimes}_{k} S$ are open immersions for any $k^{\prime} / k$.

See $\S 1.3$ for the definition of pseudo-separatedness. Also, keep in mind that non-algebraic change of the base field introduces points not seen over the original base field, so the compatible of $S^{>h}$ and $S^{\geq h}$ with respect to such extension on $k$ does require a proof.

Proof. Once $S^{>h}$ and $S^{\geq h}$ are proved to be admissible, their formation clearly commutes with base change on $S$. By fpqc descent theory for admissible opens [C3, Lemma 4.2.4] and fpqc descent for properties of morphisms [C3, Thm. 4.2.7] (applied to the properties of being an open immersion and being quasi-compact), we may work fpqc-locally on $S$ for both the admissibility and the compatibility with change in the base field. Thus, by Corollary 2.2.5 and arguments on the algebraic side (or, alternatively, by Theorems 2.2.3, 2.2.4, and 3.2.1), we may suppose that $E$ admits a $\Gamma_{1}(N)$-structure where $N$ is divisible by an integer $d \geq 5$ that is a unit in $R$. The moduli stack $\mathscr{M}_{\Gamma_{1}(N) / R}$ is therefore a proper and finitely presented scheme over $R$, and by Theorem 3.2.3 the analytification $\mathscr{M}_{\Gamma_{1}(N) / k}^{\text {an }}$ of its generic fiber represents the moduli functor of $\Gamma_{1}(N)$-structures on generalized elliptic curves over rigid-analytic spaces over $k$. Thus, it suffices to treat the universal object over $\mathscr{M}_{\Gamma_{1}(N) / k}^{\text {an }}$.

More generally, avoiding the specificity of modular curves, it suffices to consider the case of the analytified generic fiber of a generalized elliptic curve $\mathscr{E} \rightarrow \mathscr{M}$ over a proper, flat, and finitely presented $R$-scheme. The significance of properness over $R$ is that if $\widehat{\mathscr{E}} \rightarrow \widehat{\mathscr{M}}$ is the induced map of formal completions along an ideal of definition of $R$ then in the commutative diagram

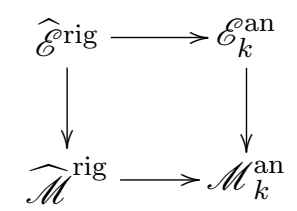

the horizontal maps are isomorphisms [C1, 5.3.1(4)]. This gives a link between the rigid-analytic setup over $k$ and an $R$-structure on relative formal groups that will tie in with Lubin's integral theory (applied to formal models of rigid-analytic fibers over the base space $\left.\mathscr{M}_{k}^{\text {an }}\right)$. 
The map $\widehat{\mathscr{E}} \rightarrow \widehat{\mathscr{M}}$ is formally smooth along the identity section $\widehat{e}$, so $\widehat{e}^{*} \widehat{\Omega}_{\overparen{\mathscr{E}}}^{1} \widehat{\mathscr{M}}$ is an invertible sheaf on $\widehat{\mathscr{M}}$. We may therefore find a covering of $\widehat{\mathscr{M}}$ by formal open affines $\operatorname{Spf} A$ on which this invertible sheaf has trivial restriction, and the associated rigid spaces (Spf $A)^{\text {rig }}$ are an admissible covering of $\widehat{\mathscr{M}}^{\text {rig }}=\mathscr{M}_{k}^{\text {an }}$ (and continue to be so after any analytic extension on $k$ ). Fix such an $A$. The formal completion $\Gamma$ of $\widehat{\mathscr{E}}$ along its identity section is a smooth formal group over $\widehat{\mathscr{M}}$, and $\Gamma_{A}=\left.\Gamma\right|_{\operatorname{Spf} A}$ has globally free relative cotangent space along $\widehat{e}_{A}=\left.\widehat{e}\right|_{\operatorname{Spf} A}$. Let $I$ be the augmentation ideal in $\mathscr{O}\left(\Gamma_{A}\right)$, so $I$ is free of rank 1 as an $\mathscr{O}\left(\Gamma_{A}\right)$-module. Using a formal coordinate $X$ that is dual to a generating cotangent vector along $\widehat{e}_{A}$ (i.e., $X$ is a generator of $I$ ), we obtain an identification $\Gamma_{A} \simeq \operatorname{Spf}(A \llbracket X \rrbracket)$ as formal $\operatorname{Spf}(A)$-schemes, where $A \llbracket X \rrbracket$ has the $(\pi, X)$-adic topology for any nonzero $\pi \in \mathfrak{m}_{R}$. We thereby obtain on $A \llbracket X \rrbracket$ the structure of a 1-parameter commutative formal group law over the $\mathbf{Z}_{p}$-algebra $A$.

If $a_{\Gamma_{A}}$ is the coefficient of $X^{p}$ in $[p]^{*}(X)$ then $a_{\Gamma_{A}}$ depends on $X$ but for any $h \in[1 / p, 1) \cap \sqrt{ }\left|k^{\times}\right|$the admissible locus $\left|a_{\Gamma_{A}}\right|>h$ on $(\operatorname{Spf} A)^{\text {rig }}=\operatorname{Sp}\left(k \otimes_{R} A\right)$ is independent of $X$ and so is intrinsic to $\Gamma_{A}$. The same goes for the locus $\left|a_{\Gamma_{A}}\right| \geq h$ if $h \in(1 / p, 1] \cap \sqrt{ }\left|k^{\times}\right|$. By Lemma A.1.5, for every $s \in \mathscr{M}_{k}^{\text {an }}=\widehat{\mathscr{M}}^{\text {rig }}$ there exists an $A$ as above such that $s=\widehat{s}^{\text {rig }}$ for a suitable continuous map $\widehat{s}: A \rightarrow R^{\prime}$ to the valuation ring $R^{\prime}$ of a finite extension $k^{\prime} / k$. The crux of the matter is that $E_{s}=\widehat{\mathscr{E}}_{s}^{\text {rig }}$ has semi-stable reduction with associated formal group given by formal completion of the formal proper $\operatorname{Spf}\left(R^{\prime}\right)$-scheme $\widehat{\mathscr{E}}_{\widehat{s}}$ along its identity section.

We conclude from the definition of the Hasse invariant $h\left(E_{s}\right)$ via $\S \mathrm{B} .3$ that $h\left(E_{s}\right)=\max \left(1 / p,\left|a_{\Gamma_{A}}(s)\right|\right)$. Thus, $S^{>h}$ meets $(\operatorname{Spf} A)^{\mathrm{rig}}$ in exactly the domain $\left|a_{\Gamma_{A}}\right|>h$, and similarly with non-strict inequalities for $S \geq h$. This proves the admissibility of $S^{>h}$ and $S^{\geq h}$, and also shows that $S^{\geq h} \rightarrow S$ is quasi-compact and that $\left\{S^{\geq h}\right\}_{h>h_{0}}$ is an admissible cover of $S^{>h_{0}}$ for any $h_{0} \in[1 / p, 1) \cap \sqrt{ }\left|k^{\times}\right|$. From our local description of $S^{>h}$ and $S^{\geq h}$ we see that the formation of each is compatible with arbitrary analytic extension on $k$.

Example 4.1.2. Let $\Gamma=\Gamma(N)$ (resp. $\left.\Gamma_{1}(N)\right)$ with $N \geq 3$ (resp. $N \geq 5$ ). Let $\mathscr{E} \rightarrow \mathscr{M}$ be universal $\Gamma$-structure in the category of $k$-schemes, so $\mathscr{M}$ is proper over Spec $k$. By Theorem 3.2.3, $\mathscr{E}^{\text {an }} \rightarrow \mathscr{M}^{\text {an }}$ is the universal $\Gamma$-structure in the category of rigid-analytic spaces over $k$, and the universality is preserved under arbitrary analytic extension on $k$.

For $h \in[1 / p, 1) \cap \sqrt{ }\left|k^{\times}\right|$, it follows from Theorem 4.1.1 that $\left(\mathscr{M}^{\text {an }}\right)^{>h}$ (equipped with the restriction of $\mathscr{E}$ an over it) represents the functor of $\Gamma$-structures on rigidanalytic generalized elliptic curves whose fibers have Hasse invariant $>h$, and similarly for $\left(\mathscr{M}^{\text {an }}\right) \geq h$ when $h \in(1 / p, 1] \cap \sqrt{ }\left|k^{\times}\right|$. These constructions respect arbitrary analytic extension on $k$. 
Theorem 4.1.3. Let $E \rightarrow S$ be a generalized elliptic curve over a rigid-analytic space over $k$, and fix $h \in\left[p^{-p /(p+1)}, 1\right) \cap \sqrt{ }\left|k^{\times}\right|$. There exists a unique finite étale $S^{>h}$-group $G^{>h}$ equipped with a closed immersion of $S^{>h}$-groups $\left.G^{>h} \hookrightarrow E^{\mathrm{sm}}\right|_{S>h}$ such that $G_{s}^{>h} \hookrightarrow E_{s}^{\mathrm{sm}}$ is the canonical subgroup for all $s \in S^{>h}$. Moreover, $G^{>h}$ is Zariski-open in $\left.E^{\mathrm{sm}}[p]\right|_{S>h}$ and Zariski-closed in $\left.E\right|_{S>h}$. The same holds over $S^{\geq h}$ for any $h \in\left(p^{-p /(p+1)}, 1\right] \cap \sqrt{ }\left|k^{\times}\right|$.

The formation of $G^{>h}$ and $G^{\geq h}$ is compatible with base change on $S$ and (for quasi-separated or pseudo-separated $S$ ) with change of the base field.

Proof. Throughout the argument, when working with $S^{\geq h}$ we assume $p^{-p /(p+1)}<$ $h \leq 1$. Let us first check uniqueness. We may assume that $S=S^{>h}$ (resp. $S=S^{\geq h}$ ), and then we can assume that $S$ is connected. Suppose that $G \hookrightarrow E^{\mathrm{sm}}$ and $H \hookrightarrow E^{\mathrm{sm}}$ satisfy the given conditions to be $G^{>h}$ (resp. $G^{\geq h}$ ), so they are closed in the separated flat $S$-group $E^{\mathrm{sm}}[p]$. Since we are in characteristic 0, consideration of fibers shows that $E^{\mathrm{sm}}[p]$ is étale over $S$. Thus, for uniqueness it suffices to prove the general claim that if $Y$ is étale and separated over a connected rigid space $S$, and if $Z, Z^{\prime} \rightrightarrows Y$ are closed immersions such that $Z$ and $Z^{\prime}$ are finite and étale over $S$, then an equality of fibers $Z_{s_{0}}=Z_{s_{0}}^{\prime}$ inside of $Y_{s_{0}}$ for a single $s_{0} \in S$ forces an equality $Z=Z^{\prime}$ inside of $Y$.

The maps $Z, Z^{\prime} \rightrightarrows Y$ must be finite étale (since $Y$ is separated and étale over $S$ ), so $Z \cap Z^{\prime}=Z \times_{Y} Z^{\prime}$ is also $S$-finite and $S$-étale. Hence, we may replace $Z^{\prime}$ with $Z \cap Z^{\prime}$ to reduce to proving that if $i: Z^{\prime} \hookrightarrow Z$ is a closed immersion between finite étale $S$-spaces with $Z_{s_{0}}^{\prime}=Z_{s_{0}}$ for some $s_{0} \in S$ then $i$ is an isomorphism. Connectivity of $S$ forces $Z_{s}$ and $Z_{s}^{\prime}$ to have ranks that are independent of $s$, so taking $s=s_{0}$ shows that these ranks agree. Hence, $i_{s}: Z_{s}^{\prime} \hookrightarrow Z_{s}$ must be an isomorphism for all $s \in S$. The map $i$ between finite étale $S$-spaces must be finite and étale, and since $i_{s}$ is an isomorphism for all $s \in S$ it follows that $i$ has constant degree 1 and hence it is an isomorphism.

Uniqueness implies compatibility with base change, and in particular with finite extension of the base field. Let us now check compatibility with arbitrary change of the base field (granting existence) for quasi-separated or pseudo-separated $S$. Since the formation of $S^{>h}$ and $S^{\geq h}$ is compatible with extension of the base field, we may replace $S$ with $S^{>h}$ or $S^{\geq h}$ to reduce to the case when $S=S^{>h}$ or $S=S^{\geq h}$ respectively (a property that is preserved under extension on $k$, by Theorem 4.1.1). We may assume that $S$ is connected, so by [C1, 3.2.3] there exists a finite extension $K / k$ such that $K \widehat{\otimes}_{k} S$ has finitely many connected components and all are geometrically connected over $K$. Thus, we can assume that $S$ is geometrically connected over $k$. Let $k^{\prime} / k$ be an analytic extension field, and suppose that canonical subgroups $G \hookrightarrow E^{\mathrm{sm}}$ and $G^{\prime} \hookrightarrow E^{\prime \text { sm }}$ exist, where $E^{\prime}=$ $k^{\prime} \widehat{\otimes}_{k} E$. Since $S^{\prime}=k^{\prime} \widehat{\otimes}_{k} S$ is connected, we may therefore use the method of proof of uniqueness to show that the two finite étale closed $S^{\prime}$-subgroups $k^{\prime} \widehat{\otimes}_{k} G$ and 
$G^{\prime}$ inside of $E^{\prime s m}[p]$ agree, since they certainly agree in fibers at any single point of $S^{\prime}$ induced by a point of $S$. In particular, $k^{\prime} \widehat{\otimes}_{k} G$ has the canonical subgroup property inside of $E^{\prime s m}$.

Continuing to assume existence, the étale property for $E^{\mathrm{sm}}[p]$ ensures that $G$ must be Zariski-open in $E^{\mathrm{sm}}[p]$. Indeed, the closed immersion $G \rightarrow E^{\mathrm{sm}}[p]$ over $S$ between $S$-étale spaces must be an étale map, and clearly an étale closed immersion is a Zariski-open immersion. Also, since $G$ is $S$-finite and $E$ is $S$ separated, the $S$-map $G \rightarrow E$ is finite and hence (by Nakayama's Lemma) it is a closed immersion since it is obviously so on fibers over $S$. This is a special case of the general fact that proper rigid-analytic monomorphisms are closed immersions.

It remains to prove existence, and by fpqc descent theory (for morphisms and objects finite over the base) the uniqueness allows us to work fpqc-locally. The same technique as in the proof of Theorem 4.1.1 therefore allows us to reduce to treating the loci $\left(\mathscr{M}_{k}^{\text {an }}\right)^{>h}$ and $\left(\mathscr{M}_{k}^{\text {an }}\right)^{\geq h}$ for $\mathscr{E}_{k}^{\text {an }} \rightarrow \mathscr{M}_{k}^{\text {an }}$, where $\mathscr{E} \rightarrow \mathscr{M}$ is a generalized elliptic curve over a proper, flat, and finitely presented $R$-scheme $\mathscr{M}$. More specifically, we may assume that this arises by local base change from a family over $\mathbf{Z}_{p}$ (such as a universal family over a modular curve). Thus, we can suppose that $R$ is a discrete valuation ring provided that we prove more: we must directly prove in this special situation that the construction we shall give for the canonical subgroup "works" after any further analytic extension on $k$ (so as to return to the initial situation for which the ground field was perhaps not discretely-valued).

The properness hypothesis on $\mathscr{M}$ provides the identification $\widehat{\mathscr{M}}^{\text {rig }}=\mathscr{M}_{k}^{\text {an }}$ (and likewise for $\mathscr{E}$ ), and so we may find a finite (necessarily admissible) covering of $\mathscr{M}_{k}^{\text {an }}$ by affinoids $(\operatorname{Spf} A)^{\text {rig }}=\operatorname{Sp}\left(k \otimes_{R} A\right)$ for formal ( $R$-flat) open affines Spf $A \subseteq \widehat{\mathscr{M}}$ such that $\widehat{e}^{*} \widehat{\Omega}_{\overparen{E} / \widehat{\mathscr{M}}}^{1}$ is trivial. We let $\Gamma$ denote the formal completion of $\widehat{\mathscr{E}}$ along its identity section, so its restriction $\Gamma_{A}$ over $\operatorname{Spf} A$ has the form $\operatorname{Spf}(A \llbracket X \rrbracket)$ as a pointed formal scheme (where $A \llbracket X \rrbracket$ has the $(\pi, X)$-adic topology for any nonzero $\pi \in \mathfrak{m}_{R}$ ). Fixing the choice of $X$, we define $a \in A$ to be the coefficient of $X^{p}$ in $[p]^{*}(X)$, so $\max (|a(s)|, 1 / p)$ is the Hasse invariant $h\left(E_{s}\right)$ for all $s \in S=(\operatorname{Spf} A)^{\text {rig }}$.

By Theorem 3.2.6, the canonical map of (separated) rigid-analytic spaces $\Gamma^{\text {rig }} \rightarrow \widehat{\mathscr{E}}^{\text {rig }}=\mathscr{E}_{k}^{\text {an }}$ is an open immersion over the base $\mathscr{M}_{k}^{\text {an }}$ and remains so after arbitrary analytic extension on $k$. By construction, this map lands inside of the relative smooth locus of the target and so it gives rise to an open immersion $\Gamma^{\text {rig }} \hookrightarrow\left(\mathscr{E}_{k}^{\text {an }}\right)^{\mathrm{sm}}$ that is clearly a map of $\mathscr{M}_{k}^{\text {an }}$-groups. We shall now restrict this map over the affinoid $S=\operatorname{Sp}\left(k \otimes_{R} A\right)$ for $A$ as above, and we will inspect what happens over $S^{>h}$ and $S^{\geq h}$. 
Let $\Gamma_{S}$ denote the $S$-group $\Gamma_{A}^{\mathrm{rig}}$, so $\Gamma_{S}=S \times \Delta$ as pointed $S$-spaces. Define $G_{S}^{>h}=\Gamma_{S}[p] \cap W^{>h}$, where

$$
W^{>h}=\left\{(s, x) \in S \times\left.\Delta|| a(s)|>h,| a(s)|| x\right|^{p-1} \leq 1 / p\right\}
$$

lies over $S^{>h}$. We define $W^{\geq h}$ and $G_{\bar{S}}^{\geq h}=\Gamma_{S}[p] \cap W^{\geq h}$ over $S^{\geq h}$ similarly. Observe that the formation of $G_{S}^{>h}$ and $G_{\bar{S}}^{\geq h}$ is compatible with arbitrary extension on $k$, due to the preservation of the "open immersion" conditions in Theorem 3.2.6 under any analytic extension on the base field. Also, the respective canonical maps from $G_{S}^{>h}$ and $G_{\bar{S}}^{\geq h}$ to $\left.\mathscr{E}_{k}^{\text {an }}\right|_{S>h}$ and $\mathscr{E}_{k}$ an $\left.\right|_{S \geq h}$ are clearly monomorphisms and remain so after any analytic extension of the base field.

The conditions in the definition of $W^{>h}$ force $|x|$ to be uniformly bounded away from 1 (explicitly, $|x|<(p h)^{-1 /(p-1)}$ ) at all points $(s, x) \in W^{>h}$, so it is obvious that $W^{>h} \rightarrow S^{>h}$ is a quasi-compact morphism; the same goes for the morphism $W^{\geq h} \rightarrow S^{\geq h}$. Since $\Gamma^{\mathrm{rig}}[p] \rightarrow \Gamma^{\mathrm{rig}}$ is a closed immersion (as $\Gamma^{\mathrm{rig}}$ is separated over the base), it follows that the maps $G_{S}^{>h} \rightarrow S^{>h}$ and $G_{\bar{S}}^{\geq h} \rightarrow S^{\geq h}$ are quasicompact. But $W^{>h}$ and $W^{\geq h}$ are admissible opens in $\Gamma_{S}$, so $G_{S}^{>h}$ and $G_{\bar{S}}^{\geq h}$ are admissible opens in $\Gamma_{S}[p]$. Since the multiplication map $[p]: \Gamma^{\mathrm{rig}} \rightarrow \Gamma^{\mathrm{rig}}$ for the smooth commutative $\mathscr{M}_{k}^{\text {an }}$-group $\Gamma^{\text {rig }}$ is an étale map, as $k$ has characteristic 0 , it follows that the torsion subgroup $\Gamma_{S}[p]$ is étale over $S$. Thus, $G_{S}^{>h}$ and $G_{\bar{S}}^{\geq h}$ are étale over $S$, and hence are respectively étale over $S^{>h}$ and $S^{\geq h}$. By Lubin's theory on fibers (see $\S$ B.3), $\left(G_{S}^{>h}\right)_{s} \subseteq E_{s}^{\mathrm{sm}}$ is the canonical subgroup for all $s \in S^{>h}$, and similarly for $\left(G_{\bar{S}}^{\geq h}\right)_{s}$ if $s \in S^{\geq h}$; this is where we use the condition $|a(s)||x|^{p-1} \leq 1 / p$ in the definitions of $W^{>h}$ and $W^{\geq h}$.

The maps $G_{S}^{>h} \rightarrow S^{>h}$ and $G_{\bar{S}}^{\geq h} \rightarrow S^{\geq h}$ have been shown to be quasi-compact, separated, and étale, with fibers of constant rank (namely, $p$ ). Thus, by Theorem A.1.2 it follows that both maps are finite. In particular, the respective monomorphisms $\left.G_{S}^{>h} \rightarrow \mathscr{E}_{k}^{\text {an }}\right|_{S>h}$ and $\left.G_{\bar{S}}^{\geq h} \rightarrow \mathscr{E}_{k}^{\text {an }}\right|_{S \geq h}$ are closed immersions, and likewise $\left.G_{S}^{>h} \rightarrow \Gamma_{S}[p]\right|_{S>h}$ and $\left.G_{S}^{\geq h} \rightarrow \Gamma_{S}[p]\right|_{S \geq h}$ are closed immersions. Since finite étale covers always split over a finite étale cover the base, we may use connectivity arguments (for closed immersions of finite étale spaces into the separated étale $S$-group $\Gamma_{S}[p]$ restricted over $S^{>h}$ or $S^{\geq h}$ ) as in the proof of uniqueness to infer that the finite étale $G_{S}^{>h}$ inside of $\left.\left(\mathscr{E}_{k}^{\text {an }}\right)^{\mathrm{sm}}\right|_{S>h}$ is an $S^{>h}$-subgroup (as this is true on fibers over $S^{>h}$ ), and similarly for $G_{\bar{S}}^{\geq h}$ over $S^{\geq h}$. Thus, we have proved existence over $S^{>h}$ and $S^{\geq h}$. By uniqueness, these local constructions for the admissible collection of affinoids $S$ covering $\mathscr{M}_{k}^{\text {an }}$ glue to give global existence over $\left(\mathscr{M}_{k}^{\text {an }}\right)^{>h}$ and $\left(\mathscr{M}_{k}^{\text {an }}\right)^{\geq h}$.

Finally, we must explain why this construction over $\mathscr{M}_{k}^{\text {an }}$ is compatible with arbitrary analytic change in the base field. The content of this claim is that for each $A$ as above we have a compatibility between the formal parameter $X$ and 
formal parameters on fibral integral formal groups associated to fibers over points $s^{\prime} \in k^{\prime} \widehat{\otimes}_{k} \operatorname{Sp}\left(k \otimes_{R} A\right)=\left(\operatorname{Spf}\left(R^{\prime} \widehat{\otimes}_{R} A\right)\right)^{\text {rig }}$ at which the Hasse invariant exceeds $h$ or is $\geq h$, exactly as in the proof of Theorem 4.1.1. This compatibility follows from the construction of $X$ in terms of $\widehat{\mathscr{E}}$ and $\widehat{\mathscr{M}}$.

Example 4.1.4. Consider the Tate curve $\underline{\text { Tate }}_{d}^{\text {an }} \rightarrow \Delta$ as in $\S 3.1$. The fiber over the origin is not smooth, and the other fibers all have toric reduction (by (3.1.4)), so $\Delta=\Delta^{\geq 1}$ for this family. Hence, the preceding general theory provides a canonical subgroup $G$ in $\left(\underline{\operatorname{Tate}}_{d}^{\text {an }}\right)^{\operatorname{sm}}[p]$ over the entire base $\Delta$. Let us make it explicit, with the expected answer on fibers over $\Delta-\{0\}$.

By $[\mathrm{C} 2,(2.5 .3) f f$.$] , in the formal Tate curve \widehat{\text { Tate }}_{d}$ over $\mathbf{Z} \llbracket q^{1 / d} \rrbracket$ there is a unique open formal torus

$$
T=\operatorname{Spf}\left(\mathbf{Z} \llbracket q^{1 / d} \rrbracket\{\{t, 1 / t\}\}\right) \hookrightarrow \widehat{\text { Tate }}_{d}
$$

lifting the identity component of the standard $d$-gon fiber over $q^{1 / d}=0$. This lies inside of the formal smooth locus as an open subgroup, so applying the functor $(\cdot)^{\text {rig }}$ (as in Theorem 3.1.5 with $C=\mathbf{Z}$ ) gives an open immersion over $\Delta$

$$
\{|t|=1\} \times \Delta=T^{\mathrm{rig}} \hookrightarrow{\widehat{\mathrm{Tate}_{d}}}_{d}^{\mathrm{rig}}=\underline{\text { Tate }}_{d}^{\mathrm{an}},
$$

and this factors through the $\Delta$-smooth locus $\left(\operatorname{Tate}_{d}^{\text {an }}\right)^{\text {sm }}$ as a map of $\Delta$-groups that (by (3.1.4)) is the canonical map $\mu_{p} \rightarrow \mathbf{G}_{m}^{\text {an }} / q_{0}^{d} \mathbf{Z}$ over any $q_{0} \in \Delta-\{0\}$. In particular, we get an closed immersion of $\Delta$-groups $T[p]^{\text {rig }}=T^{\text {rig }}[p]=\mu_{p} \times \Delta \hookrightarrow$ $\left(\operatorname{Tate}_{d}^{\text {an }}\right)^{\mathrm{sm}}$. The finite étale $\Delta$-groups $T[p]^{\mathrm{rig}}$ and $G$ inside of the separated étale $\Delta$-group $\left(\text { Tate }_{d}^{\text {an }}\right)^{\mathrm{sm}}[p]$ clearly agree over the origin, so by connectivity of $\Delta$ they are equal everywhere.

4.2. Higher torsion-level canonical subgroups. In [Bu, $\S 3-\S 4]$, Buzzard uses results of Lubin to study a theory of higher torsion-level canonical subgroups in the universal elliptic curves over certain rigid-analytified modular curves (extending earlier work of Katz and Gouvea). That development is given over modular curves over finite extensions of $\mathbf{Q}_{p}$, and it rests on the fine structure of such curves. We wish to explain how the methods in $\S 4.1$ may be used to obtain such a theory for generalized elliptic curves over arbitrary rigid spaces over arbitrary analytic extension fields $k / \mathbf{Q}_{p}$. The definition we use is not the same as that of Buzzard and Gouvea, but the equivalence of the definitions (for elliptic curves) will be proved below; an advantage of our definition is that it readily generalizes to the case of abelian varieties [C4]. In the 1-dimensional case we shall obtain explicit optimal universal bounds on the Hasse invariant that recover the ad hoc ones used in $[\mathrm{Bu}, \S 3]$; in the higher-dimensional case it does not seem reasonable to expect that necessary and sufficient conditions for the existence canonical subgroups are given by bounds on a Hasse invariant as in the case of relative dimension 1 . 
We continue to normalize the absolute value on $k$ by requiring $|p|=1 / p$. Let $E$ be a rigid-analytic generalized elliptic curve over $k$, and let $k^{\prime} / k$ be a finite extension as in the semistable reduction theorem (Theorem B.3.2): the algebraization of $E_{k^{\prime}}$ (under GAGA and Corollary 2.1.14) extends to a generalized elliptic curve $E^{\prime}$ over the valuation ring $R^{\prime}$ of $k^{\prime}$. (In the discussion that follows, it is immaterial whether $E$ begins life as an algebro-geometric or rigid-analytic object; the relativization below, however, will only make sense in the rigid-analytic setting.) The associated $p$-divisible formal group $\widehat{E}^{\prime}$ over $R^{\prime}$ is independent of the choice of $E^{\prime}$, and in Definition B.3.5 we use an arbitrary choice of formal parameter for this formal group to define an intrinsic notion of size for a point of $\widehat{E}^{\prime}$ valued in the maximal ideal of the valuation ring of any analytic extension field of $k^{\prime}$. In particular, for any $0<r<1$ we get a $k$-subgroup $E^{\mathrm{sm}}\left[p^{n}\right]_{\leq r}^{0}$ in $E^{\mathrm{sm}}\left[p^{n}\right]$ whose geometric points are precisely those of $\widehat{E}^{\prime}\left[p^{n}\right]$ with size $\leq r$. (The superscript " 0 " in this notation is meant to be suggestive of an identity component for a finite flat group scheme over $R^{\prime}$.)

Remark 4.2.1. Elementary arguments with formal group laws show that the subgroup of points $x$ of $\widehat{E}^{\prime}$ with size $<p^{-1 /(p-1)}=\left|\zeta_{p}-1\right|$ has no nontrivial $p$-torsion and so is torsion-free, and that if $\operatorname{size}(x) \geq p^{-1 /(p-1)}$ then $\operatorname{size}([p](x)) \leq \operatorname{size}(x)^{p}$, so by induction on $n \geq 1$ if $r<p^{-1 / p^{n-1}(p-1)}=\left|\zeta_{p^{n}}-1\right|$ then $E^{\mathrm{sm}}\left[p^{n}\right]_{<r}^{0}$ is killed by $p^{n-1}$.

Definition 4.2.2. A level-n canonical subgroup of $E$ is a $k$-subgroup $G_{n} \subseteq$ $E^{\mathrm{sm}}\left[p^{n}\right]$ of the form $E^{\mathrm{sm}}\left[p^{n}\right]_{\leq r_{n}}^{0}$ such that its group of geometric points is free of rank 1 as a $\mathbf{Z} / p^{n} \mathbf{Z}$-module.

Geometrically, a level- $n$ canonical subgroup is a group of $p^{n}$ geometric points of $E^{\mathrm{sm}}\left[p^{n}\right]$ whose members are nearer to the origin than the other $p^{n}$-torsion points, and whose module structure is free. The specific radius $r_{n}$ in Definition 4.2.2 is not unique, but if such a radius exists then Remark 4.2.1 implies that necessarily $r_{n} \geq p^{-1 / p^{n-1}(p-1)}=\left|\zeta_{p^{n}}-1\right|$. Obviously if a level- $n$ canonical subgroup $G_{n}$ exists in $E$ then it is unique and $G_{n}\left[p^{m}\right]$ is a level- $m$ canonical subgroup for all $1 \leq m \leq n$.

Remark 4.2.3. In the case of an elliptic curve $E$ our definition of level- $n$ canonical subgroup is different from the one used in $[\mathrm{Bu}]$ and $[\mathrm{G}]$, but the equivalence of these definitions follows from Theorem 4.2.5.

It is clear that the existence or not of a level- $n$ canonical subgroup $G_{n}$ is unaffected by extension on $k$, and that the formation of $G_{n}$ commutes with any analytic extension of the base field. If $E$ has (potentially) good reduction, then the notion of a level-1 canonical subgroup coincides with the notion of canonical subgroup in the work of Katz. In particular, a level-1 canonical subgroup exists in a generalized elliptic curve $E$ over $k$ if and only if $h(E)>p^{-p /(p+1)}$. 
Example 4.2.4. If $E$ has potentially ordinary or potentially toric reduction then it admits a level- $n$ canonical subgroup for all $n \geq 1$. This subgroup consists of the $p^{n}$-torsion geometric points of the height-1 multiplicative formal group $\widehat{E}^{\prime}$ over the valuation ring of a sufficiently large finite extension $k^{\prime} / k$. Thus, as in the case $n=1$, the interesting case in the theory is when $E$ is an elliptic curve with potentially supersingular reduction.

We first formulate an existence result that is analogous to Lubin's input into Katz' work for $n=1$, and then we shall prove a relativization generalizing Theorem 4.1.3.

Theorem 4.2.5. A rigid-analytic generalized elliptic curve $E$ over $k$ admits a level-n canonical subgroup $G_{n}$ if and only if $h(E)>p^{-p / p^{n-1}(p+1)}$. Moreover, if $E$ is an elliptic curve and such $a G_{n}$ exists with $n>1$ then $G_{n} / G_{n}[p]$ is a level$(n-1)$ canonical subgroup in the elliptic curve $E / G_{n}[p]$ and $h\left(E / G_{n}[p]\right)=h(E)^{p}$.

In particular, $E$ admits a level-n canonical subgroup for all $n \geq 1$ if and only if $h(E)=1$, which is to say that $E$ has potentially toric or potentially ordinary reduction.

Proof. By replacing $k$ with a finite extension, we may assume that $E$ (viewed algebraically) extends to a generalized elliptic curve $\mathscr{E}$ over the valuation ring $R$ of $k$. First consider the cases of potentially ordinary or potentially toric reduction, which is to say that the formal group $\widehat{\mathscr{E}}$ has height 1 , or in other words that the semi-abelian scheme $c(\mathscr{E})^{\mathrm{sm}}$ given by the $R$-smooth locus of the contraction away from the identity section has closed fiber that is a torus or an ordinary elliptic curve. In this case $h(E)=1$ and $\widehat{\mathscr{E}}\left[p^{n}\right]_{k}$ is a level- $n$ canonical subgroup of $E^{\text {sm }}$ for all $n$. When $E$ is moreover an elliptic curve, it is obvious that $G_{n} / G_{n}[p]$ is a level- $(n-1)$ canonical subgroup of $E / G_{n}[p]$.

We now may and do assume $\mathscr{E}$ is an elliptic curve over $R$ whose reduction is supersingular (so $h(E)<1$ ). The case $n=1$ is discussed in $\S$ B.3, so we now proceed via induction on $n$ and assume $n>1$. First assume that $E$ admits a level- $n$ canonical subgroup $G_{n}$, and let $G_{m}=G_{n}\left[p^{m}\right]$ be the level- $m$ canonical subgroup of $E$ for $1 \leq m \leq n$. In particular, since $E$ has a level-1 canonical subgroup we must have $p^{-p /(p+1)}<h(E)<1$. Let $\mathscr{G}_{1}$ be the local schematic closure of the level-1 canonical subgroup $G_{1}$ in $\mathscr{E}$, so $\mathscr{E} / \mathscr{G}_{1}$ is the unique elliptic curve over $R$ with analytic $k$-fiber $E / G_{1}$ (by GAGA over $k$ and Lemma B.3.1 over $R$ ). By studying the degree- $p$ isogeny of elliptic curves $\mathscr{E} \rightarrow \mathscr{E} / \mathscr{G}_{1}$ over $R$ we shall prove that $G_{n} / G_{1}$ is a level- $(n-1)$ canonical subgroup of $E / G_{1}$, so in particular the level-1 canonical subgroup of $E / G_{1}$ has preimage $G_{2}$ that is cyclic of order $p^{2}$ and so is not $E[p]$. Granting this for a moment, it then would follow from results of Lubin (see [Bu, Thm. 3.3(ii)-(iv)]) that $h(E)>p^{-1 /(p+1)}$ and 
$h\left(E / G_{1}\right)=h(E)^{p}$, but induction gives $h\left(E / G_{1}\right)>p^{-p / p^{n-2}(p+1)}$ so we would get $h(E)>p^{-p / p^{n-1}(p+1)}$ as desired.

Let us now check that indeed $G_{n} / G_{1}$ is a level- $(n-1)$ canonical subgroup of $E / G_{1}$. Clearly it is free of rank 1 over $\mathbf{Z} / p^{n-1} \mathbf{Z}$. Since $\left(E / G_{1}\right)\left[p^{n-1}\right] \subseteq E\left[p^{n}\right] / G_{1}$, our problem is to analyze size in the formal group of $\mathscr{E} / \mathscr{G}_{1}$ for the images of the points of $E\left[p^{n}\right] / G_{1}$. This problem is intrinsic to the height-2 $p$-divisible formal groups $\Gamma=\widehat{\mathscr{E}}$ and $\Gamma^{\prime}=\Gamma / \mathscr{G}_{1}$. The following argument could probably be expressed entirely in the language of Newton polygons for $\left[p^{m}\right]^{*}$ on 1-parameter commutative formal groups, but we prefer to use a more geometric language that carries over to the higher-dimensional case in [C4].

Let $X$ be a formal parameter for $\Gamma$, so $\mathscr{O}(\Gamma) \simeq R \llbracket X \rrbracket$ is finite free as a module over the subring $\mathscr{O}\left(\Gamma^{\prime}\right)$ for which a formal parameter is given by

$$
X^{\prime}=\mathrm{N}_{\Gamma / \Gamma^{\prime}}(X)=\prod_{\xi \in G_{1}}(X-X(\xi))=X \cdot \prod_{\xi \in G_{1}-\{0\}}(X-X(\xi)) .
$$

If $x$ is a generator of the cyclic group $G_{n}$ then $\left[p^{n-1}\right](x)$ is a generator of $G_{1}$ and so has size $(p h(E))^{-1 /(p-1)}>p^{-1 /(p-1)}$ (whence $x$ has size $\left.>p^{-1 /(p-1)}\right)$. But if a point $x_{0}$ of the formal group $\Gamma$ has size $r \geq p^{-1 /(p-1)}$ then $[p]\left(x_{0}\right)$ has size $\leq r^{p}$ (Remark 4.2.1), so inductively we conclude that $\left[p^{j}\right](x)$ has size at most $\operatorname{size}(x)^{p^{j}}$ for $1 \leq j \leq n-1$. Hence, $|X(x)| \geq(p h(E))^{-1 / p^{n-1}(p-1)}$, and since $n>1$ this strictly exceeds $|X(\xi)|=(p h(E))^{-1 /(p-1)}$ for all $\xi \in G_{1}-\{0\}$. It follows via (4.2.1) that $x \bmod G_{1}$ in $E / G_{1}$ has size $\left|X^{\prime}(x)\right|$ that is equal to $|X(x)|^{p}$.

To conclude that $G_{n} / G_{1}$ is level- $(n-1)$ canonical subgroup in $E / G_{1}$, we have to prove that any point in $\left(E / G_{1}\right)\left[p^{n-1}\right]$ not in $G_{n} / G_{1}$ has size $>|X(x)|^{p}$. For $x^{\prime} \in E$ lifting such a point, we have $\left[p^{n-1}\right]\left(x^{\prime}\right) \in G_{1}=p^{n-1}\left(G_{n}\right)$, so $x^{\prime}=x_{1}^{\prime}+x_{0}^{\prime}$ with $x_{1}^{\prime} \in G_{n}$ and $x_{0}^{\prime} \in E\left[p^{n-1}\right]$ with $x_{0}^{\prime}$ not in $G_{n}$. By the defining property of $G_{n}$ as a level- $n$ canonical subgroup, the $p^{n}$-torsion point $x_{0}^{\prime}$ in $E$ has size strictly larger than that of any point of $G_{n}$, so in particular it has larger size than $x_{1}^{\prime}$ and larger size than any $\xi \in G_{1}-\{0\}$. Since the formal group law has integral coefficients and its linear part is addition, we conclude

$$
\left|X\left(x^{\prime}\right)\right|=\left|X\left(x_{0}^{\prime}\right)\right|>|X(x)|,\left|X\left(x^{\prime}\right)-X(\xi)\right|=\left|X\left(x_{0}^{\prime}\right)\right|>|X(x)|
$$

for all $\xi \in G_{1}-\{0\}$. This implies $\left|X^{\prime}\left(x^{\prime}\right)\right|=\left|X\left(x_{0}^{\prime}\right)\right|^{p}>|X(x)|^{p}$, as desired.

It remains to prove that if $h(E)>p^{-p / p^{n-1}(p+1)}$ then $E$ admits a level- $n$ canonical subgroup. We may assume $n>1$ and that the result is known for smaller positive values of $n$; as we have seen above, we can also assume that $E$ extends to an elliptic curve over $R$ with supersingular reduction (so in particular $h(E)<1)$. Since $h(E)>p^{-p /(p+1)}$, certainly $E$ admits a level-1 canonical subgroup $G_{1}$. Since $n \geq 2$ we have $h(E)>p^{-1 /(p+1)}$, so by $[\mathrm{Kz}, 3.10 .7(2)]$ it follows that $h\left(E / G_{1}\right)=h(E)^{p}>p^{-p / p^{(n-1)-1}(p+1)}$, and by induction $E / G_{1}$ 
therefore has a level-( $(n-1)$ canonical subgroup $G_{n-1}^{\prime}$. Letting $G_{n}$ be its preimage in $E$, we shall prove that $G_{n}$ is level- $n$ canonical. Certainly $G_{n}$ has order $p^{n}$, and to see that it is a free $\mathbf{Z} / p^{n} \mathbf{Z}$-module we just have to rule out the possibility that it contains $E[p]$, which is to say that $E[p] / G_{1}$ is the $p$-torsion in $G_{n-1}^{\prime}$. But $G_{n-1}^{\prime}[p]$ is the level-1 canonical subgroup of $E / G_{1}$, so the module structure of $G_{n}$ will work out provided that $E[p] / G_{1}$ is not the level-1 canonical subgroup of $E / G_{1}$. The nonzero points in the level-1 canonical subgroup of $E / G_{1}$ have size $\left(p h\left(E / G_{1}\right)\right)^{-1 /(p+1)}=p^{-1 /(p+1)} h(E)^{-p /(p+1)}$, so to show that this subgroup does not equal $E[p] / G_{1}$ it is enough to show that nonzero points in $E[p] / G_{1}$ have size in $E / G_{1}$ that exceeds this value. A nonzero point in $E[p] / G_{1}$ is represented by a point $x^{\prime} \in E[p]-G_{1}$, so its size in $E$ is $h(E)^{1 /\left(p^{2}-p\right)}$ and this exceeds the size in $E$ of all points in $G_{1}$. Thus, by (4.2.1) the image of $x^{\prime}$ in $E / G_{1}$ has size equal to $\operatorname{size}_{E}\left(x^{\prime}\right)^{p}=h(E)^{1 /(p-1)}$. Our problem is therefore to show $h(E)^{p /(p-1)+1 /(p-1)}>p^{-1 /(p-1)}$. But $h(E) \in\left(p^{-1 /(p+1)}, 1\right)$ since $n \geq 2$, so the desired inequality is clear. This shows that $G_{n}$ as just defined is cyclic of order $p^{n}$.

Finally, we have to prove that if $n>1$ then the points of $G_{n}$ have strictly smaller size than all other $p^{n}$-torsion points in $E$. Pick $x^{\prime} \in E\left[p^{n}\right]$ not in $G_{n}$, so there is a largest $0 \leq m \leq n-1$ such that $\left[p^{m}\right]\left(x^{\prime}\right) \notin G_{n}$. Since $\operatorname{size}\left(x^{\prime}\right) \geq$ size $\left(\left[p^{m}\right]\left(x^{\prime}\right)\right)$, we can replace $x^{\prime}$ with $\left[p^{m}\right]\left(x^{\prime}\right)$ and so we can assume $x^{\prime} \notin G_{n}$ and $[p]\left(x^{\prime}\right) \in G_{n}$. Thus, $[p]\left(x^{\prime}\right) \in E\left[p^{n-1}\right] \cap G_{n}=G_{n}\left[p^{n-1}\right]=p \cdot G_{n}$ (as we have already proved that $G_{n}$ is free of rank 1 over $\mathbf{Z} / p^{n} \mathbf{Z}$ ), so $x^{\prime}=x_{0}^{\prime}+x_{1}^{\prime}$ with $x_{0}^{\prime} \in G_{n}$ and $x_{1}^{\prime} \in E[p]-G_{1}$. It is therefore enough to show that $x_{1}^{\prime}$ has size strictly exceeding the size of any point in $G_{n}$ (as then $x^{\prime}$ has the same size as $x_{1}^{\prime}$, so we are done). Renaming $x_{1}^{\prime}$ as $x^{\prime}$ therefore reduces us to the case $x^{\prime} \in E[p]-G_{1}$, so $\operatorname{size}\left(x^{\prime}\right)=h(E)^{1 /\left(p^{2}-p\right)}$. Our problem is to prove $\operatorname{size}_{E}(x)<h(E)^{1 /\left(p^{2}-p\right)}$ for all $x \in G_{n}$, and since $G_{n}$ is cyclic it suffices to treat the case when $x$ is a generator of $G_{n}$. We shall now restrict our attention to such points $x$.

Since $n>1, G_{1}=p^{n-1} \cdot G_{n} \subseteq p \cdot G_{n}$ consists of points with size strictly smaller than the size of the generator $x$ of $G_{n}$, so by (4.2.1) we deduce

$$
\operatorname{size}_{E / G_{1}}\left(x \bmod G_{1}\right)=\operatorname{size}_{E}(x)^{p} .
$$

Our desired inequality is thus $\operatorname{size}_{E / G_{1}}\left(x \bmod G_{1}\right) \stackrel{?}{<} h(E)^{1 /(p-1)}$. But $x \bmod G_{1}$ lies in the level- $(n-1)$ canonical subgroup $G_{n-1}^{\prime}$ in $E / G_{1}$, so its size (in $E / G_{1}$ ) is strictly less than that of the $p$-torsion in $E / G_{1}$ not contained in the level-1 canonical subgroup of $E / G_{1}$. That is,

$$
\operatorname{size}_{E / G_{1}}\left(x \bmod G_{1}\right)<h\left(E / G_{1}\right)^{1 /\left(p^{2}-p\right)}=h(E)^{1 /(p-1)} .
$$


Corollary 4.2.6. If $E$ is a generalized elliptic curve over $k$ and $h(E)>p^{-p / p^{n-1}(p+1)}$ then the generators of the level-n canonical subgroup $G_{n}$ in $E$ have size $p^{-1 / p^{n-1}(p-1)}$ $h(E)^{-1 /(p-1)}$.

Proof. In the case $n=1$ this is part of Lubin's theory (as in $\S$ B.3), so suppose $n>1$. Let $G_{m}$ be the level- $m$ canonical subgroup in $E$ for $1 \leq m \leq n$, so $E / G_{1}$ has $G_{n} / G_{1}$ as its level- $(n-1)$ canonical subgroup by Theorem 4.2.5. Pick a generator $x \in G_{n}$, so $(4.2 .2)$ gives $\operatorname{size}_{E / G_{1}}\left(x \bmod G_{1}\right)=\operatorname{size}_{E}(x)^{p}$. By by Theorem 4.2.5 and induction $\operatorname{size}_{E / G_{j}}\left(x \bmod G_{j}\right)=\operatorname{size}_{E}(x)^{p^{j}}$ for $1 \leq j \leq n-1$. Since $x \bmod G_{n-1}$ generates the subgroup $G_{n} / G_{n-1}$ that is the level-1 canonical subgroup of $E / G_{n-1}$ with $h\left(E / G_{n-1}\right)=h(E)^{p^{n-1}}$ by Theorem 4.2 .5 , the settled case of level 1 gives

$$
\begin{aligned}
\operatorname{size}_{E}(x)^{p^{n-1}} & =\operatorname{size}_{E / G_{n-1}}\left(x \bmod G_{n-1}\right) \\
& =\left(p h\left(E / G_{n-1}\right)\right)^{-1 /(p-1)} \\
& =\left(p h(E)^{p^{n-1}}\right)^{-1 /(p-1)} .
\end{aligned}
$$

Extracting $p^{n-1}$ th roots gives the result.

Here is the relativization for higher torsion levels:

Theorem 4.2.7. Fix $n \geq 1$. Let $E \rightarrow S$ be a generalized elliptic curve over a rigid-analytic space over $k$, and fix $h \in\left[p^{-p / p^{n-1}(p+1)}, 1\right) \cap \sqrt{ }\left|k^{\times}\right|$. There exists a unique finite étale $S^{>h}$-group $G_{n}^{>h}$ equipped with a closed immersion of $S^{>h}$ groups $\left.G_{n}^{>h} \hookrightarrow E^{\mathrm{sm}}\right|_{S>h}$ such that $G_{n, s}^{>h} \hookrightarrow E_{s}^{\mathrm{sm}}$ is the level-n canonical subgroup for all $s \in S^{>h}$. Moreover, $G_{n}^{>h}$ is Zariski-open in $\left.E^{\mathrm{sm}}[p]\right|_{S>h}$ and Zariski-closed in $\left.E\right|_{S>h}$. The same holds over $S^{\geq h}$ for any $h \in\left(p^{-p / p^{n-1}(p+1)}, 1\right] \cap \sqrt{ }\left|k^{\times}\right|$.

The formation of $G_{n}^{>h}$ and $G_{\bar{n}}^{\geq h}$ is compatible with base change on $S$ and (for quasi-separated or pseudo-separated $S$ ) with change of the base field.

Proof. The proof is identical to that of Theorem 4.1.3 (which is the case $n=1$ ), except for two changes: we use the inequality $|a(s)||x|^{p-1} \leq p^{-1 / p^{n-1}}$ in the definitions of $W^{>h}$ and $W^{\geq h}$, and we replace references to Lubin's work with references to Theorem 4.2.5 and Corollary 4.2.6.

Example 4.2.8. The same argument as in Example 4.1.4 shows that for any $d \geq 1$ and $n \geq 1$, the level- $n$ canonical subgroup in $\underline{T a t e}_{d}^{a n} \rightarrow \Delta$ is the canonically embedded $\Delta$-subgroup $\mu_{p^{n}} \times \Delta$.

Example 4.2.9. Let $N$ be a positive integer not divisible by $p$ and let $E \rightarrow X$ be the universal rigid-analytic generalized elliptic curve over $X=X_{1}\left(N p^{m}\right)_{/ \mathbf{Q}_{p}}^{\mathrm{an}}$, with $m \geq 1$ and $N p^{m} \geq 5$ (so the $\Gamma_{1}\left(N p^{m}\right)$-moduli problem is representable over $\operatorname{Sp}\left(\mathbf{Q}_{p}\right)$; see Theorem 3.2.3). Let $C \hookrightarrow E^{\mathrm{sm}}\left[p^{m}\right]$ be the universal finite 
étale cyclic subgroup of order $p^{m}$ in the $\Gamma_{1}\left(N p^{m}\right)$-level structure over $X$ (so $C$ is Zariski-closed in $E$ and both Zariski-open and Zariski-closed in $E^{\mathrm{sm}}\left[p^{m}\right]$ ). Choose $h \in\left[p^{-1 / p^{m-1}(p+1)}, 1\right) \cap p^{\mathbf{Q}}$ (resp. $\left.h \in\left(p^{-1 / p^{m-1}(p+1)}, 1\right] \cap p^{\mathbf{Q}}\right)$ and let $S=X^{>h}$ (resp. $S=X^{\geq h}$ ). The restriction $E_{S}=\left.E\right|_{S}$ has a level-m canonical subgroup $G_{m}\left(E_{S}\right) \subseteq E_{S}^{\mathrm{sm}}\left[p^{m}\right]$ that is a finite étale open and closed subgroup with order $p^{m}$ and cyclic geometric fibers.

By the method of proof of uniqueness in Theorem 4.1.3, the condition of equality for $G_{m}\left(E_{S}\right)_{/ S^{\prime}}$ and $C_{/ S^{\prime}}$ inside of $E_{/ S^{\prime}}$ for rigid spaces $S^{\prime}$ over $S$ is represented by a Zariski-open and Zariski-closed locus in $S$ (i.e., a union of connected components of $S$ ); in particular, if the geometric fiber of $C$ over a single point $s \in S$ is the level- $m$ canonical subgroup in the geometric fiber of $E$ at $s$ then $C$ coincides with $G_{m}\left(E_{S}\right)$ over the entire connected component of $s$ in $S$. This criterion is especially convenient when taking $s$ to be a cusp or ordinary point on the modular curve $X$, and the theory of integral models for modular curves over $p$-adic integer rings ensures that every connected component of $S$ contains a cusp and an ordinary point. (A more general result concerning distinguished points on connected components of rigid-analytic curves is proved in [C4] and plays a crucial role in the higher-dimensional theory of canonical subgroups of any level.)

Example 4.2.10. Choose $n \geq 1$ and $r \in \sqrt{ }\left|k^{\times}\right|$with $p^{-1 / p^{n-1}(p-1)}<r<1$. Let $E \rightarrow S$ be a generalized elliptic curve and suppose $h\left(E_{s}\right)>p^{-p / p^{n-1}(p+1)}$ for all $s \in S$, so $E$ admits a relative level- $n$ canonical subgroup $G_{n}$ over $S$. Define

$$
h_{n, p}(r)= \begin{cases}r^{p^{2}-p} \in\left[p^{-1 / p^{n-1}(p+1)}, 1\right) & \text { if } p^{-1 / p^{n-1}\left(p^{2}-1\right)} \leq r<1, \\ (1 / r)^{p-1} p^{-1 / p^{n-1}} \in\left(p^{-1 / p^{n-1}(p+1)}, 1\right) & \text { otherwise. }\end{cases}
$$

Using Corollary 4.2.6 and the formula $h\left(E_{s}\right)^{1 /\left(p^{2}-p\right)}$ for the size of $p$-torsion points in $E_{s}$ not in the level-1 canonical subgroup when $h\left(E_{s}\right)<1$, it is easy to check that $G_{n, s}=E_{s}^{\mathrm{sm}}\left[p^{n}\right]_{\leq r}^{0}$ if and only if $s \in S^{>h_{n, p}(r)}\left(\right.$ resp. $s \in S^{\geq h_{n, p}(r)}$ ) when $r \geq p^{-1 / p^{n-1}\left(p^{2}-1\right)}$ (resp. $\left.r<p^{-1 / p^{n-1}\left(p^{2}-1\right)}\right)$. In particular, for $r$ sufficiently close to the theoretical minimum $p^{-1 / p^{n-1}(p-1)}$ in Remark 4.2 .1 it follows that for all $s \in S^{\geq h_{n, p}(r)}$ the fibral level- $n$ canonical subgroup is $E_{s}^{\mathrm{sm}}\left[p^{n}\right]_{\leq r}^{0}$; the point is that we are controlling the size of points of the level- $n$ canonical subgroup subject to only a universal lower bound $h_{n, p}(r)$ on $h\left(E_{s}\right)$. In the higher-dimensional case with fixed $n$ and $p$ and with $r$ sufficiently near $p^{-1 / p^{n-1}(p-1)}$, an analogous universal "size bound" $A\left[p^{n}\right]_{\leq r}^{0}$ for level- $n$ canonical subgroups is proved in [C4] for $g$-dimensional abelian varieties $A$ with "Hasse invariant" $h(A) \geq h_{n, p, g}(r)$; we do not know an explicit formula for such an $h_{n, p, g}(r)$ when $g>1$.

4.3. Frobenius lifts. Let $E$ be a generalized elliptic curve over $k$ with $h(E)>$ $p^{-p / p^{n-1}(p+1)}$, so $E$ admits a level- $n$ canonical subgroup. Replace $k$ with a finite extension so that (by Theorem B.3.2) $E$ extends to a generalized elliptic curve $\mathscr{E}$ over $R$ whose associated formal group $\widehat{\mathscr{E}}$ "is" a $p$-divisible group over $R$ that is 
intrinsic to $E$. We may form the finite flat schematic closure $\mathscr{G}_{n}$ of $G_{n}$ in the finite flat closed $R$-subgroup $\widehat{\mathscr{E}}\left[p^{n}\right] \subseteq \mathscr{E}\left[p^{n}\right]$ and consider its mod-pR reduction $\overline{\mathscr{G}}_{n}$ as a finite flat closed $R / p R$-subgroup in $\overline{\mathscr{E}}=\mathscr{E} \bmod p R$. How does $\overline{\mathscr{G}}_{n}$ relate to the kernel $\operatorname{ker}\left(F_{\overline{\mathscr{E}}, n, R / p R}\right)$ of the $n$-fold relative Frobenius (for $\overline{\mathscr{E}}$, or equivalently for its $p$-divisible formal group)?

If $h(E)=1$ then these two $R / p R$ subgroups coincide for all $n$. Also, if we take $c \in \mathfrak{m}_{R}$ satisfying

$$
\begin{aligned}
|c| \geq p^{-1 / p^{n-1}(p-1)} h(E)^{-1 /(p-1)} & \in\left(p^{-1 / p^{n-1}\left(p^{2}-1\right)}, p^{-1 / p^{n-1}(p-1)}\right] \\
& \subseteq\left(p^{-1 / p^{n-1}\left(p^{2}-1\right)}, p^{-1 /(p-1)}\right]
\end{aligned}
$$

(so $c \mid p$ in $R$ ) then Corollary 4.2 .6 yields equality of these two subgroup schemes modulo $c R$ for the trivial reason that the monic degree- $p^{n g}$ Weierstrass polynomial cutting out $\mathscr{G}_{n}$ in the formal group $R \llbracket X \rrbracket$ has all roots in $c R$. But by using geometry we can do better at the expense of being non-explicit, as follows. For any $\lambda \in(0,1)$, we say that two finite flat closed subschemes of $\widehat{\mathscr{E}}$ coincide modulo $p^{\lambda}$ (or modulo $p^{\lambda} R$ ) if they agree modulo $c^{\prime} R^{\prime}$ for any analytic extension $k^{\prime} / k$ and any $c^{\prime}$ in its valuation ring $R^{\prime}$ such that $\left|c^{\prime}\right| \geq p^{-\lambda}$.

Theorem 4.3.1. Fix $p$ and $n \geq 1$, and choose $\lambda \in(0,1) \cap \mathbf{Q}$. With notation as above, there exists $h_{n, p}(\lambda) \in\left(p^{-p / p^{n-1}(p+1)}, 1\right)$ such that if $h(E)>h_{n, p}(\lambda)$ then

$$
\mathscr{G}_{n} \bmod p^{\lambda} R=\operatorname{ker}\left(F_{\widehat{\mathscr{E}} \bmod p^{\lambda} R, n, R / p^{\lambda} R}\right)
$$

inside $\widehat{\mathscr{E}}\left[p^{n}\right] \bmod p^{\lambda} R$.

The proof gives a way to compute the optimal $h_{n, p}(\lambda)$ in terms of powerseries coefficients of $\left[p^{n}\right]^{*}(X)$ for the universal deformation of the unique height-2 commutative formal group of dimension 1 over $\overline{\mathbf{F}}_{p}$. However, we have not carried out such an explicit analysis. A non-explicit result similar to Theorem 4.3.1 is proved in the higher-dimensional case in [C4], and the geometric argument at the heart of the proof of Theorem 4.3.1 is written so that it applies to the higher-dimensional case verbatim.

Proof. Pick an integer $N \geq 3$ not divisible by $p$. We can restrict attention to the case $h(E)<1$, so after making a finite extension on $k$ we may assume that $E$ extends to an elliptic curve $\mathscr{E}$ over $R$ (with supersingular reduction) and that $\mathscr{E}$ admits a full level- $N$ structure. Let $Y=Y(N) / \mathbf{z}_{p}$ and let $E \rightarrow Y$ be the universal elliptic curve. Passing to the $p$-adic completions, by Corollary 3.2.7 the map $\widehat{E}^{\text {rig }} \rightarrow \widehat{Y}^{\text {rig }}$ is an elliptic curve over a rigid space over $\mathbf{Q}_{p}$ obtained by pullback along the quasi-compact open immersion $\widehat{Y}^{\text {rig }} \rightarrow Y_{\mathbf{Q}_{p}}^{\text {an }}$. Our elliptic curve $\mathscr{E}$ arises as a fiber in this family after applying the extension of scalars $k / \mathbf{Q}_{p}$, and so it suffices to find an $h_{n, p}(\lambda)$ that works for the fibers over $\widehat{Y}^{\text {rig }}$ and continues to work after any analytic extension of the base field. More generally, it suffices 
to do the same for $\mathfrak{E}^{\text {rig }} \rightarrow \mathfrak{Y}^{\text {rig }}$ with $\mathfrak{E} \rightarrow \mathfrak{Y}$ any fixed formal elliptic curve over a formal scheme $\mathfrak{Y}$ that is topologically of finite type over $\mathbf{Z}_{p}$. Working with formal schemes and Raynaud (or Berthelot) generic fibers gives a way to relate integral structure to rigid spaces.

To analyze the situation in a structural manner that avoids unnecessary use of special features of elliptic curves or modular curves (and so will be applicable in the higher-dimensional case in [C4]), let us now pass to a more general problem for formal abelian schemes. We first explain the setup (to be applied with $\mathfrak{E} \rightarrow \mathfrak{Y}$ as above for the purposes of the proof of Theorem 4.3.1). Let $\mathfrak{A} \rightarrow \mathfrak{M}$ be a formal abelian scheme of relative dimension $g$ over a formal scheme $\mathfrak{M}$ that is topologically of finite type over a discrete valuation $\operatorname{ring} R$ with fraction field $k$ that is an analytic extension of $\mathbf{Q}_{p}$; impose the normalization condition $|p|=1 / p$. Let $A \rightarrow M$ be the associated rigid-analytic map, and let $A_{0} \rightarrow M_{0}$ denote the associated abelian scheme modulo $p$. For each $m \in M=\mathfrak{M}^{\text {rig }}$ we have $m=\mathfrak{m}^{\text {rig }}$ for a unique map $\mathfrak{m}: \operatorname{Spf}(R(m)) \rightarrow \mathfrak{M}$ valued in the valuation ring $R(m)$ of $k(m)$, so we may let $\mathfrak{A}_{m}$ denote the formal abelian scheme $\mathfrak{m}^{*}(\mathfrak{A})$ over $R(m)$ whose Raynaud generic fiber over $k(m)$ is $A_{m}$. We say $m$ is ordinary if the reduction of $\mathfrak{A}_{m}$ over the residue field of $R(m)$ is an ordinary abelian variety. The locus of non-ordinary points for $A_{0} \rightarrow M_{0}$ is a Zariski-closed set $Z_{0}$ given by the vanishing of the locally principal determinant ideal of the map of rank- $g$ vector bundles

$$
\operatorname{Lie}\left(V_{A_{0} / M_{0}}\right): \operatorname{Lie}\left(A_{0}\right)^{(p)} \rightarrow \operatorname{Lie}\left(A_{0}\right)
$$

induced by the Verschiebung for $A_{0} \rightarrow M_{0}$, so the locus of ordinary points in $M$ is the quasi-compact admissible open preimage of $M_{0}-Z_{0}$ under the reduction map of sets $\mathfrak{M} \rightarrow M_{0}$. Although a local generator $H$ for this ideal may not globalize, its absolute value $|H|$ does globalize and hence loci such as $M^{\geq h}=\{|H| \geq h\}$ and $M^{>h}=\{|H|>h\}$ are admissible opens in $M$. The ordinary locus is $\{|H|=1\}$. (In the motivating example with a formal elliptic curve, such an $H$ is locally given by an $X^{p}$-coefficient as in the proof of Theorem 4.1.1. Over $M^{>p^{-p / p^{n-1}(p+1)}}$ there is a level- $n$ canonical subgroup, the fibral points of which have physical reduction 0 in the fibers of the given formal elliptic curve.)

We assume that for some $h \in(1 / p, 1) \cap p^{\mathbf{Q}}$ there is given a finite étale subgroup $\left.G \subseteq A\left[p^{n}\right]\right|_{M \geq h}$ whose geometric fibers are $\left(\mathbf{Z} / p^{n} \mathbf{Z}\right)^{g}$. Let $\widehat{\mathfrak{A}}$ denote the formal completion of $\mathfrak{A}$ along its identity section. Since $k$ is discretely-valued, by Theorem 3.2.6 there is a natural admissible open subgroup (using Berthelot's functor) $\widehat{\mathfrak{A}}^{\text {rig }} \hookrightarrow \mathfrak{A}^{\text {rig }}=A$ and this relativizes the formal groups of the formal abelian scheme fibers $\mathfrak{A}_{m}$. Intuitively, $\widehat{\mathfrak{A}}^{\text {rig }}$ is a relative open unit polydisc that glues the integral formal groups of the fibers $A_{m}=\mathfrak{A}_{m}^{\text {rig }}$. We assume that $\left.G \rightarrow A\right|_{M \geq h}$ factors through the open subgroup $\left.\left.\widehat{\mathfrak{A}}^{\text {rig }}\right|_{M \geq h} \subseteq A\right|_{M \geq h}$; equivalently, when points of $G_{m}$ are extended to integral points of $\mathfrak{A}_{m}$ for $m \in M^{\geq h}$ then such extended integral points are assumed to reduce to the identity. In particular, for all ordinary 
points $m \in M$ the fibral subgroup $G_{m} \subseteq A_{m}\left[p^{n}\right]$ is the generic fiber of the identity component "canonical subgroup" $\mathfrak{A}_{m}\left[p^{n}\right]^{0} \subseteq \mathfrak{A}_{m}\left[p^{n}\right]$. Under these assumptions, we claim that for any $\lambda \in(0,1) \cap p^{\mathbf{Q}}$ there exists $h(\lambda) \in(h, 1) \cap p^{\mathbf{Q}}$ depending on $\mathfrak{A}$ and $G$ such that if $m \in M$ satisfies $|H(m)|>h(\lambda)$ then the schematic closure $\mathscr{G}_{m}$ of $G_{m}$ in the finite flat $R(m)$-group $\mathfrak{A}_{m}\left[p^{n}\right]^{0}$ coincides modulo $p^{\lambda}$ with the kernel of the $n$-fold relative Frobenius on $\mathfrak{A}_{m} \bmod p^{\lambda}$. We also claim that this property of $h(\lambda)$ persists after any analytic extension of the base field.

The key to the proof is to extend $G \subseteq A\left[p^{n}\right]$ to a finite flat formal subgroup scheme of $\mathfrak{A}\left[p^{n}\right]$ with connected fibers, and to do this we will have to change the formal model $\mathfrak{M}$ without affecting the rigid-analytic Raynaud generic fiber. The need to possibly change $\mathfrak{M}$ is the reason that we have axiomatized our setup beyond the initial specific case of interest. By pullback to the closed formal subscheme of $\mathfrak{M}$ defined by the coherent ideal of $p$-power torsion in the structure sheaf $\mathscr{O}_{\mathfrak{M}}$, we may and do assume $\mathfrak{M}$ is $R$-flat. By [BL1, Lemma 4.4], there is an admissible formal blow-up $\mathfrak{M}^{\prime}$ of $\mathfrak{M}$ such that $M^{\geq h}$ arises from an open formal subscheme of $\mathfrak{M}^{\prime}$. By pulling back $\mathfrak{A}$ to this open formal subscheme, we may put ourselves in the case that $M=M^{\geq h}$, so $G$ now exists over the entirety of $M$. The following lemma is the crux:

Lemma 4.3.2. There is an admissible formal blow-up $\mathfrak{M}^{\prime} \rightarrow \mathfrak{M}$ such that the pullback $\mathfrak{A}^{\prime}$ of $\mathfrak{A}$ admits a finite locally free subgroup $\mathfrak{G}^{\prime} \subseteq \mathfrak{A}^{\prime}\left[p^{n}\right]$ with generic fiber $G \subseteq A\left[p^{n}\right]$ and with connected fibers over $\mathfrak{M}^{\prime}$.

We avoid noetherian hypotheses on $R$ in the proof.

Proof. We identify $\mathfrak{A}\left[p^{n}\right]$ with a finite locally free $\mathscr{O}_{\mathfrak{M}}$-algebra $\mathscr{P}$. For an open affine $\mathfrak{U}=\operatorname{Spf}(C)$ in $\mathfrak{M}$ with generic fiber $U \subseteq M$ the restriction of $\mathfrak{A}\left[p^{n}\right]$ over $\mathfrak{U}$ is associated to a finite locally free $C$-algebra $P$, and $\left.G\right|_{U}$ corresponds to a quotient of $P_{k}=k \otimes_{R} P$ by an ideal whose saturation $I$ in $P$ defines an $R$-flat quotient $P / I$ that is $C$-finite but possibly not $C$-flat. By [BL1, Lemma 1.2(c)], the ideal $I$ is finitely generated in $P$. Thus, for any $c \in C$ the quotient of $P_{\{c\}}=C_{\{c\}} \widehat{\otimes}_{C} P$ by the ideal $I_{\{c\}}=C_{\{c\}} \widehat{\otimes}_{C} I$ is $R$-flat with generic fiber given by the restriction of $G$ over the locus $|c|=1$ in $U$. Hence, $I_{\{c\}}$ is the saturated ideal associated to $G$ and $\mathfrak{U}_{\{c\}}$. It follows that the formation of $I$ globalizes over $\mathfrak{M}$ to a coherent ideal sheaf $\mathscr{I} \subseteq \mathscr{P}$ over $\mathfrak{M}$ with $R$-flat quotient algebra $\mathscr{P} / \mathscr{I}$ whose associated topologically finitely presented and closed formal subscheme $\mathfrak{G} \hookrightarrow \mathfrak{A}\left[p^{n}\right]$ is an $R$-flat and $\mathfrak{M}$-finite formal model for $G \hookrightarrow A\left[p^{n}\right]$. However, $\mathfrak{G}$ is probably not $\mathfrak{M}$-flat and so it may not be a subgroup of $\mathfrak{A}\left[p^{n}\right]$.

If $\mathfrak{M}^{\prime} \rightarrow \mathfrak{M}$ is any admissible formal blow-up, then the pullbacks of $\mathfrak{A}$ and $\mathfrak{A}\left[p^{n}\right]$ over $\mathfrak{M}^{\prime}$ are their own strict transforms since $\mathfrak{A}$ and $\mathfrak{A}\left[p^{n}\right]$ are flat over the $R$-flat $\mathfrak{M}$. Hence, the strict transform $\mathfrak{G}^{\prime}$ of $\mathfrak{G}$ under such a blow-up is a closed formal subscheme of $\mathfrak{A}^{\prime}\left[p^{n}\right]$. Raynaud's flat models theorem [BL2, 5.2] provides such an 
admissible formal blow-up for which $\mathfrak{G}^{\prime}$ is also $\mathfrak{M}^{\prime}$-flat. Hence, by renaming $\mathfrak{M}^{\prime}$, $\mathfrak{A} \times \mathfrak{M}_{\mathfrak{M}} \mathfrak{M}^{\prime}$, and $\mathfrak{G}^{\prime}$ as $\mathfrak{M}, \mathfrak{A}$, and $\mathfrak{G}$ respectively, we arrive at the situation in which the $\mathfrak{M}$-finite formal closed subscheme $\mathfrak{G}$ in $\mathfrak{A}\left[p^{n}\right]$ is $\mathfrak{M}$-flat. Since the generic fiber $G$ of $\mathfrak{G}$ is an $M$-subgroup of $A\left[p^{n}\right]$, it follows from the $R$-flatness of $\mathfrak{M}$ that $\mathfrak{G}$ is an $\mathfrak{M}$-subgroup of $\mathfrak{A}\left[p^{n}\right]$.

The flatness of $\mathfrak{G}$ over $\mathfrak{M}$ implies that for any $m \in M$ with associated point $\mathfrak{m}: \operatorname{Spf}(R(m)) \rightarrow \mathfrak{M}$, the pullback $\mathfrak{m}^{*}(\mathfrak{G})$ is a finite flat $R(m)$-subgroup of $\mathfrak{A}_{m}\left[p^{n}\right]$ and so it is the schematic closure of its generic fiber $G_{m} \hookrightarrow A_{m}\left[p^{n}\right]$. Our initial assumptions on $G$ imply that each fiber $G_{m}$ consists of points that extend to integral points of the connected finite flat $R(m)$-group $\mathfrak{A}_{m}\left[p^{n}\right]^{0}$, so the schematic closure $\mathfrak{m}^{*}(\mathfrak{G})$ is necessarily connected.

The properties in Lemma 4.3.2 imply that the map $\mathfrak{G} \rightarrow \mathfrak{A}$ physically lands in the closed identity section on underlying topological spaces (as this holds on fibers over closed points of the Jacobson space $\mathfrak{M})$. Hence, $\mathfrak{G}$ factors throught the formal completion $\widehat{\mathfrak{A}}$ along the identity section as $\mathfrak{M}$-groups. Since the composite

$$
\mathfrak{G} \rightarrow \widehat{\mathfrak{A}} \rightarrow \mathfrak{A}
$$

is a closed immersion of locally noetherian formal schemes and the second map is separated (in the sense of such formal schemes), the first map is a closed immersion. We may work locally on the quasi-compact $\mathfrak{M}$, so we can assume that $\mathfrak{M}=\operatorname{Spf}(C)$ is affine and that the tangent space for $\mathfrak{A}$ along its identity section is a free $C$-module. Thus, we can identify the formal completion of $\widehat{\mathfrak{A}}$ along its identity section with $\operatorname{Spf}\left(C \llbracket X_{1}, \ldots, X_{g} \rrbracket\right)$, using the $(\mathscr{I}, \underline{X})$-adic topology on $C \llbracket \underline{X} \rrbracket$ with $\mathscr{I}$ an ideal of definition of $C$ (such as $p C$ ). The determinant ideal for the Lie algebra map of the relative Verschiebung of $A_{0}=\mathfrak{A} \bmod p R$ therefore has a principal generator in $C / p C$ that we may lift to some $H \in C$, and the closed immersion $\mathfrak{G} \hookrightarrow \widehat{\mathfrak{A}}$ is cut out by an ideal $I \subseteq C \llbracket X_{1}, \ldots, X_{g} \rrbracket$ that is contained in the augmentation ideal.

Since $C$ is noetherian, the ideal $I$ is finitely generated. Picking generators $f_{1}, \ldots, f_{N}$ of $I$, each is a formal power series with vanishing constant term and only finitely many coefficients for monomials $\prod X_{j}^{e_{j}}$ with $\max \left(e_{j}\right)<p^{n}$. Let $\left\{c_{j}\right\}_{j \in J}$ be the finitely many such coefficients that arise. For any $\lambda \in(0,1] \cap \mathbf{Q}$, the admissible open locus $M_{\lambda}=\left\{\left|c_{j}\right| \leq p^{-\lambda}\right\}_{j \in J}$ in $M$ consists of precisely the points $m=\mathfrak{m}^{\text {rig }} \in M$ for which the specialization $\mathfrak{m}^{*}(\mathfrak{G})$ of $G_{m} \subseteq A_{m}\left[p^{n}\right]$ in $\mathfrak{A}_{m}$ has reduction modulo $p^{\lambda} R$ that is killed by the $n$-fold relative Frobenius and so (for order reasons) $\mathfrak{m}^{*}(\mathfrak{G})$ is the kernel of this iterated Frobenius map on $\mathfrak{A}_{m} \bmod p^{\lambda} R$. This description clearly persists after any extension of the base field, and so we now drop the discreteness hypothesis on $k / \mathbf{Q}_{p}$.

Given $\lambda \in(0,1) \cap \mathbf{Q}$, our problem is precisely to construct $h(\lambda) \in(0,1) \cap p^{\mathbf{Q}}$ so that $\{|H| \geq h(\lambda)\} \subseteq M_{\lambda}$. Note that $\{|H|=1\}$ is the ordinary locus, and 
$\left|c_{j}(m)\right| \geq 1 / p$ for all $\underline{j}$ at ordinary points $m \in M$. Hence, by letting $f=H$ and $g=c_{j} / p$ for each of the finitely many $\underline{j}$ (considered separately) we are reduced to the following assertion in rigid geometry: if $f$ and $g$ are analytic functions on a quasi-compact rigid space $X$ and $\{|f| \geq 1\} \subseteq\{|g| \geq 1\}$ then for any $\eta \in$ $(0,1) \cap \sqrt{ }\left|k^{\times}\right|$there exists $\eta^{\prime} \in(0,1) \cap \sqrt{ }\left|k^{\times}\right|$such that $\left\{|f(x)|>\eta^{\prime}\right\} \subseteq\{|g|>\eta\}$. We fix $\eta$, and we may assume $X$ is affinoid. The restriction of $|f|$ to the affinoid $U=\{|g| \leq \eta\}$ has a finite supremum $\sigma$. (We can and do assume $U \neq \emptyset$.) The maximum modulus theorem for $\left.f\right|_{U}$ provides $u \in U$ such that $|f(u)|=\sigma$, so $\sigma<1$ since $\eta<1$. Thus, $\eta^{\prime}=\sigma$ works.

\section{Appendix A. Some Properties of Morphisms}

A.1. Properties of finite morphisms. There are two properties of finite morphisms that we require, the topological invariance of finiteness and a fibral criterion for finiteness. We do not know references in the generality used in this paper (no discreteness hypothesis on the absolute value), so we provide proofs below. Here is the topological invariance.

Theorem A.1.1. A rigid space $X$ is affinoid if and only if $X_{\mathrm{red}}$ is affinoid. In particular, a morphism $f: Y \rightarrow Z$ between rigid spaces is finite if and only if the induced morphism $Y_{\text {red }} \rightarrow Z_{\text {red }}$ is finite.

This result is well-known to experts, but we do not know a published reference with a proof. The assertion would be trivial if there were a rigid-analytic cohomological criterion for a quasi-compact and separated rigid space to be affinoid, akin to Serre's cohomological criterion for a quasi-compact and separated scheme to be affine. However, there is no such criterion because over any non-archimedean field $k$ there exists a quasi-compact separated surface that has vanishing higher coherent cohomology but is not affinoid [Liu].

Proof. We first indicate why, for any rigid space $Y$, the natural map $i: Y_{\text {red }} \rightarrow Y$ induces a "homeomorphism" of Grothendieck topologies. That is, every admissible open $U \subseteq Y_{\text {red }}$ (resp. admissible covering $\left\{U_{j}\right\}$ of such a $U$ ) has the form $i^{-1}(V)$ (resp. $\left\{i^{-1}\left(V_{j}\right)\right\}$ ) for a unique admissible open $V \subseteq Y$ (resp. admissible covering $\left\{V_{j}\right\}$ of $V$ ). This is proved by reduction to the case of affinoid $Y$ and using the definition of admissibility and the Gerritzen-Grauert description of affinoid subdomains of an affinoid in terms of rational subdomains and Weierstrass subdomains [BGR, 7.3.5/3]; the point is that the assertions to be proved are trivial for admissible opens that are rational or Weierstrass subdomains (one merely lifts the defining inequalities through the nil-thickening of affinoid algebras).

With this "homeomorphism" point settled, we may conclude that the property of a map being quasi-compact is insensitive to passing to underlying reduced spaces. Thus, if $X_{\text {red }}$ is affinoid, then $X$ is at least quasi-compact and 
quasi-separated (i.e., the diagonal morphism $X \rightarrow X \times X$ is quasi-compact). In particular, the coherent ideal sheaf $\mathscr{I}$ defining $X_{\text {red }}$ in $X$ is nilpotent. Thus, by induction on the order of nilpotence it suffices to prove that if $X$ is a quasicompact and quasi-separated rigid space and $\mathscr{I}$ is a coherent ideal on $X$ with $\mathscr{I}^{2}=0$ such that the zero space $X_{0} \hookrightarrow X$ defined by $\mathscr{I}$ is an affinoid $\operatorname{Sp}\left(A_{0}\right)$ then $X$ is affinoid.

Let $\left\{U_{i}\right\}$ be a finite admissible affinoid open covering of $X$ and let $\left\{V_{i i^{\prime} j}\right\}_{j \in J_{i, i^{\prime}}}$ be a finite admissible affinoid open covering of the quasi-compact overlap $U_{i} \cap U_{i^{\prime}}$. Consider the left exact sequence

$$
0 \rightarrow \mathscr{O}_{X}(X) \rightarrow \prod \mathscr{O}_{X}\left(U_{i}\right) \rightarrow \prod \mathscr{O}_{X}\left(V_{i i^{\prime} j}\right)
$$

The two terms on the right are naturally $k$-Banach algebras and have underlying $k$-Banach spaces of countable type. By continuity, it follows that (A.1.1) gives $A=\mathscr{O}_{X}(X)$ a structure of $k$-Banach algebra having countable type as a $k$-Banach space. We will now show that $A$ is $k$-affinoid (and hence the $k$-Banach algebra structure is the unique one on $A$ ).

Using the hypothesis that $X_{0}=\operatorname{Sp}\left(A_{0}\right)$ is $k$-affinoid and the fact that the square-zero coherent ideal sheaf $\mathscr{I}$ on $X$ may be viewed as a coherent sheaf on $X_{0}$, the ideal $I=\mathrm{H}^{0}(X, \mathscr{I})$ in $A$ is naturally a finite $A_{0}$-module and we have an exact sequence $0 \rightarrow I \rightarrow A \rightarrow A_{0} \rightarrow 0$. Choose a surjection of $k$-algebras

$$
k\left\langle\left\langle t_{1}, \ldots, t_{n}\right\rangle\right\rangle \rightarrow A_{0}
$$

with $t_{r} \mapsto \bar{a}_{r}$, where $\bar{a}_{r} \in A_{0}$ is power-bounded. Choose any $a_{r} \in A$ lifting $\bar{a}_{r}$. We claim that $a_{r}$ is power-bounded (for the above $k$-Banach algebra structure on $A$ ). By definition of the $k$-Banach structure on $A$ it suffices to check powerboundedness of each $\left.a_{r}\right|_{U_{i}} \in \mathscr{O}_{X}\left(U_{i}\right)$, so we reduce to showing that for a $k$-affinoid $B$ and a square-zero ideal $J \subseteq B$, the preimage of a power-bounded element under $B \rightarrow B / J$ is power-bounded. Power-boundedness in an affinoid is equivalent to having absolute value at most 1 at every maximal ideal, and this property is insensitive to passing to the quotient by a nilpotent ideal.

Due to the power-boundedness of the $a_{r}$ 's, we may use [BGR, 6.1.1/4] to construct a unique continuous $k$-algebra morphism $k\left\langle\left\langle t_{1}, \ldots, t_{n}\right\rangle\right\rangle \rightarrow A$ that satisfies $t_{r} \mapsto a_{r}$. This map induces the surjection (A.1.2) onto $A / I=A_{0}$. Recall that $I$ is a finite module over $A / I=A_{0}$. For $A_{0}$-module generators $b_{1}, \ldots, b_{m}$ of $I$ we have $b_{s}^{2}=0$ for all $s$, so there is a unique continuous $k$-algebra map

$$
k\left\langle\left\langle t_{1}, \ldots, t_{m}, u_{1}, \ldots, u_{m}\right\rangle\right\rangle \rightarrow A
$$

satisfying $t_{r} \mapsto a_{r}, u_{s} \mapsto b_{s}$. This map is visibly surjective, so $A=\mathscr{O}_{X}(X)$ is indeed $k$-affinoid. We conclude that there is a natural map of rigid spaces $\iota: X \rightarrow \operatorname{Sp}(A)$, but we do not yet know that it is an isomorphism. 
Since $\iota$ is compatible with the isomorphism $X_{0} \simeq \operatorname{Sp}\left(A_{0}\right)=\operatorname{Sp}(A / I)$, we conclude that $\iota$ is a homeomorphism of Grothendieck topologies and that for all $x \in X$ there is a commutative diagram of local $k$-algebras

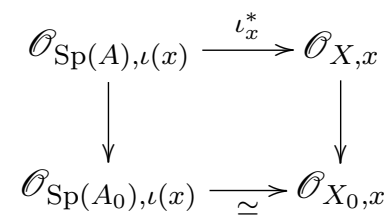

with the bottom row an isomorphism, the vertical maps surjective, and the right column having square-zero kernel generated by $I$ (as $\mathscr{I}$ may be viewed as a coherent sheaf on the affinoid $X_{0}$ and hence has its stalk modules over the stalks of $\mathscr{O}_{X_{0}}$ or $\mathscr{O}_{X}$ generated by its global sections). We conclude that the top row is surjective. Moreover, for any open affinoid $W \subseteq \iota\left(U_{i}\right)$, the preimage $\iota^{-1}(W) \simeq$ $U_{i} \times_{\mathrm{Sp}(A)} W$ is affinoid. The map $\iota$ therefore satisfies all of the criteria in [BGR, 9.5.3/5] to be a closed immersion. That is, $X$ admits a closed immersion into an affinoid and hence must be affinoid (and $\iota$ is therefore an isomorphism).

The following fibral criterion for finiteness is inspired by some results in the complex-analytic and scheme-theoretic cases.

Theorem A.1.2. Let $f: X \rightarrow Y$ be a flat map between rigid spaces. The map $f$ is finite if and only if it is quasi-compact and separated with finite fibers and its fiber-rank is locally constant on $Y$.

Remark A.1.3. This finiteness criterion was independently proved by AbbesMokrane [AM, Lemme A.1] when $k$ is discretely-valued. (The quasi-separatedness hypothesis in [AM, Lemme A.1] must be strengthened to separatedness, as this is used in the proof and the result is false without it.) Their argument follows a strategy that is similar to ours, except that they use complete local rings on formal models and we cannot use such completions because local rings on formal models are not noetherian when $k$ is not discretely-valued.

Before we begin the proof of Theorem A.1.2, we note that the complex-analytic analogue (without a quasi-compactness condition) is an immediate consequence of the structure theorem for locally quasi-finite maps in the complex-analytic case. Due to the admissibility condition for open covers in rigid geometry, we will need to work a little harder in the rigid case. The scheme-theoretic analogue of Theorem A.1.2 is given in [DR, II, 1.19] with noetherian hypotheses, proved via Zariski's Main Theorem and the valuative criterion for properness. Our proof in the rigid case will use this scheme-theoretic version via the theory of formal models, but we need to drop the noetherian condition (for applications to the case in which $k$ has non-discrete valuation). Thus, we first prove a generalization of [DR, II, 1.19]: 
Lemma A.1.4. Let $f: X \rightarrow Y$ be a flat morphism of schemes that is locally of finite presentation. The map $f$ is finite if and only if it is separated with finite fibers and its fiber-rank is locally constant on $Y$.

The application of this lemma in the proof of Theorem A.1.2 will be in the case of a map that is already known to be quasi-compact, but it is curious that this lemma does not require an assumption of quasi-compactness. In contrast, the quasi-compactness assumption in Theorem A.1.2 is necessary, as is seen by the standard example of the bijective local isomorphism $\{|t|=1\} \coprod\{|t|<1\} \rightarrow$ $\{|t| \leq 1\}$.

Proof. The "only if" implication is trivial, since a finite flat $Y$-scheme that is locally of finite presentation is classified by a quasi-coherent sheaf of $\mathscr{O}_{Y}$-algebras whose underlying $\mathscr{O}_{Y}$-module is locally free of finite rank. Thus, we focus on the "if" direction. By working locally on $Y$, we may suppose that all fibers of $f$ have the same rank, say $d$.

We first settle the case of local $Y$. By fpqc base change, we may assume that $Y$ is henselian. The structure theorem for locally quasi-finite separated maps [EGA, $\left.\mathrm{IV}_{4}, 18.5 .11(c)\right]$ ensures that we can write $X=X^{\prime} \amalg X^{\prime \prime}$ with $X^{\prime \prime}$ having empty closed fiber and $X^{\prime} \rightarrow Y$ finite. Since $X^{\prime} \rightarrow Y$ is visibly finite flat and locally of finite presentation, it is finite free of some constant rank $d^{\prime}$. Looking over the closed fiber, $d^{\prime}=d$. The open immersion $X^{\prime} \hookrightarrow X$ must therefore be an isomorphism on fibers over $Y$, and hence an isomorphism. This settles the case of local $Y$. In general, choose $y \in Y$. The map $X \times_{Y} \operatorname{Spec} \mathscr{O}_{Y, y} \rightarrow \operatorname{Spec} \mathscr{O}_{Y, y}$ is finite flat of rank $d$ by the local case, and we want to smear out this property over a neighborhood of $y$. We may assume $Y$ is affine and we may find a quasi-compact open $U \subseteq X$ that contains $X \times_{Y}$ Spec $\mathscr{O}_{Y, y} \subseteq X$. In particular, $U \times_{Y}$ Spec $\mathscr{O}_{Y, y}$ is finite locally free of rank $d$ over $\operatorname{Spec} \mathscr{O}_{Y, y}$. Since $U \rightarrow Y$ is of finite presentation, by shrinking $Y$ around $y$ we may assume that $U \rightarrow Y$ is finite locally free of rank $d$. The open immersion $U \hookrightarrow X$ is therefore an isomorphism on fibers over $Y$, and hence an isomorphism.

Lemma A.1.5. Let $R$ be the valuation ring of $k$ and let $\mathfrak{Y}$ be a formal scheme over $\operatorname{Spf}(R)$ that is flat and locally topologically of finite presentation. Every point $y: \operatorname{Sp}\left(k^{\prime}\right) \hookrightarrow \mathfrak{Y}^{\text {rig }}$ has the form $y=\mathfrak{y}^{\text {rig }}$ for a unique closed immersion

$$
\mathfrak{y}: \operatorname{Spf}(B) \hookrightarrow \mathfrak{Y},
$$

with $B$ a finite flat domain over $R$. Conversely, every closed point $y_{0} \in \mathfrak{Y}$ underlies such a morphism $\mathfrak{y}$.

Proof. This follows from the theory of rig-points as developed in [BL1], particularly [BL1, 3.5]. 
Proof. (of Theorem A.1.2). The "only if" implication is trivial, since rigid spaces equipped with a finite morphism to $Y$ are classified by coherent sheaves of $\mathscr{O}_{Y^{-}}$ algebras, so we focus on the "if" direction. Working locally on $Y$, we may assume $Y$ is affinoid, or more conceptually that $X$ and $Y$ are both quasi-compact and separated. We may likewise assume that $f$ has constant fiber rank, say $d$.

Let $\mathfrak{f}: \mathfrak{X} \rightarrow \mathfrak{Y}$ be a formal model of $f$. By [BL1, Prop. 4.7], the map $\mathfrak{f}$ is automatically separated. By [BL2, Cor. 5.3], we may choose $\mathfrak{f}$ to be quasi-finite. Since the operations of base change by any $\mathfrak{Y}^{\prime} \rightarrow \mathfrak{Y}$ and replacing $\mathfrak{X}$ by a closed formal subscheme do not destroy the quasi-finiteness or separatedness conditions on the formal side, by [BL2, Thm. 5.2] we may also assume $\mathfrak{f}$ is flat. It suffices to prove that the formal scheme map $f$ is finite, and for this it suffices to prove that the ordinary scheme maps $\mathfrak{f}_{n}=\mathfrak{f} \bmod \mathfrak{I}^{n+1}$ are finite for a fixed ideal of definition $\mathfrak{I}$ of the valuation ring $R$ of $k$ and any $n \geq 0$. A priori each $\mathfrak{f}_{n}$ is quasi-finite, separated, flat, and finitely presented. Thus, by Lemma A.1.4 it is enough to prove that each $\mathfrak{f}_{n}$ has fibers of constant rank $d$.

Let $\bar{f}$ denote the induced map of finite type schemes over the residue field $\widetilde{k}$ of $R$. It is necessary and sufficient to prove that the quasi-finite, flat, separated map $\bar{f}$ has all fibers of constant rank $d$. By [EGA, $\mathrm{IV}_{3}, 9.8 .8$ ] the locus of fibers with a specified rank is constructible, so it suffices to look at fibers over closed points. Choose a closed point $y_{0} \in \mathfrak{Y}$. By Lemma A.1.5, there exists a finite flat local $R$-algebra domain $B$ and a locally closed immersion $y: \operatorname{Spf}(B) \rightarrow \mathfrak{Y}$ that hits $y_{0}$. Let $y_{k} \in Y$ denote the corresponding rigid point, with residue field equal to the fraction field of $B$. The fibers of the $\mathfrak{f}_{n}$ 's (or, equivalently, of $\overline{\mathfrak{f}}$ ) over $y_{0}$ are identified with the fiber of

$$
\mathfrak{T} \stackrel{\text { def }}{=} \mathfrak{X} \times \mathfrak{Y} \operatorname{Spf}(B)
$$

over the residue field of $B$. The formal scheme $\mathfrak{T}$ is quasi-finite flat over $\operatorname{Spf}(B)$, and $\operatorname{Spec}(B / \mathfrak{I} B)$ consists of a single point, so by topological reasons we conclude that the $R$-flat $\mathfrak{T}$ is affine and finite over $\operatorname{Spf}(B)$, say $\mathfrak{T}=\operatorname{Spf}(C)$. Thus, $C$ is finite flat over $B$. Since $B$ is local, $C$ is finite free over $B$ of some rank $d^{\prime}$. We just need to show $d^{\prime}=d$. Since $C \otimes_{R} k$ is finite free of rank $d^{\prime}$ over the fraction field $k\left(y_{k}\right)=B \otimes_{R} k$ of $B$ and it is also the affinoid algebra of $f^{-1}\left(y_{k}\right)$, we conclude that indeed $d^{\prime}=d$.

A.2. Change of base field. Since change of the base field in rigid geometry often creates points that are not seen on the original base (due to the lack of "enough physical points" in the rigid site), it is not obvious that reasonable fibral properties of a morphism, such as having equidimensional fibers of a fixed dimension $d$, are preserved by arbitrary change of the base field. We need to know that for many reasonable properties of morphisms of quasi-separated rigid spaces there is good behavior with respect to change in the base field. Raynaud's theory of formal models is well-suited to settling such questions because base 
change for formal models is closely related to the better-behaved case of schemes (where there are enough points).

Theorem A.2.1. Let $\mathfrak{X}$ and $\mathfrak{Y}$ be formal schemes over $\operatorname{Spf}(R)$ that are locally topologically finitely presented and $R$-flat. Let $\mathfrak{f}: \mathfrak{X} \rightarrow \mathfrak{Y}$ be an $R$-morphism, and let $f=f^{\text {rig }}: X \rightarrow Y$ be the induced map between quasi-separated rigid spaces over $k$.

(1) If all fibers of $\mathfrak{f}$ have dimension $\leq n$ then all fibers of $f$ have dimension $\leq n$, and the converse holds when $\mathfrak{f}$ is flat.

(2) If $\mathfrak{f}$ is flat and surjective with all fibers geometrically reduced of pure dimension $d$, then the same holds for $f$.

Proof. We begin by proving (1). First consider the implication " $\Rightarrow$." Let $y$ : $\operatorname{Sp}\left(k^{\prime}\right) \rightarrow Y$ be a point. By Lemma A.1.5, we have $y=\mathfrak{y}^{\text {rig }}$ for a morphism $\mathfrak{y}: \operatorname{Spf}(B) \rightarrow \mathfrak{Y}$, where $B$ is a finite flat domain over $R$ with fraction field $k^{\prime}$. Using base change by $\mathfrak{y}$, we may assume $\mathfrak{Y}=\operatorname{Spf}(B)$. We may compose $\mathfrak{f}$ with the finite flat map $\operatorname{Spf}(B) \rightarrow \operatorname{Spf}(R)$ in order to reduce to the case $\mathfrak{Y}=\operatorname{Spf}(R)$. That is, for a topologically finitely presented (and not necessarily flat) formal scheme $\mathfrak{X}$ over $\operatorname{Spf}(R)$, we need to show that the "generic fiber" $X=\mathfrak{X}^{\text {rig }}$ has dimension $\leq n$ if $\mathfrak{X}$ has dimension $\leq n$.

Working locally, without loss of generality $\mathfrak{X}=\operatorname{Spf}(A)$ is affine. The fiber $\widetilde{A}=A / \mathfrak{m}_{R} A$ of $A$ over the residue field $\widetilde{k}$ of $R$ is a finite type $\widetilde{k}$-algebra of dimension $\leq n$. By Noether normalization for finitely generated algebras over a field, there is a finite map of $\widetilde{k}$-algebras $\widetilde{k}\left[x_{1}, \ldots, x_{n}\right] \rightarrow \widetilde{A}$. Let $a_{i} \in A$ lift the image of $x_{i}$ in $\widetilde{A}$. Consider the unique continuous $R$-algebra morphism

$$
R\left\langle\left\langle t_{1}, \ldots, t_{n}\right\rangle\right\rangle \rightarrow A
$$

that satisfies $t_{i} \mapsto a_{i}$. We claim that (A.2.1) is finite, in which case the $k$-affinoid $k \otimes_{R} A$ is finite over an $n$-variable Tate algebra and hence has dimension $\leq n$, as desired.

Somewhat more generally, if $\varphi: B \rightarrow A$ is a continuous $R$-algebra map between topologically finitely presented $R$-algebras, then we claim that $\varphi$ is finite if the corresponding $\widetilde{k}$-algebra map $\widetilde{\varphi}$ is finite (the converse is trivial). Let $t_{1}, \ldots, t_{n}$ be topological $R$-algebra generators of $A$. Pick $a_{1}, \ldots, a_{m} \in A$ lifting $\widetilde{B}$-module generators of $\widetilde{A}$, so there exist monic polynomials $f_{i} \in B[T]$ and an ideal of definition $\mathfrak{I}$ of $R$ such that $f_{i}\left(a_{i}\right) \in \mathfrak{I} A$ and $t_{j} \in \sum \varphi(B) a_{i}+\mathfrak{I} A$. Since $A / \mathfrak{I} A$ is generated by the $t_{j}$ 's as an $R / \mathfrak{I}$-algebra we conclude that $A / \mathfrak{I} A$ is finite over $B / \mathfrak{I} B$, but $A$ is $\mathfrak{I}$-adically separated and complete [BL1, $§ 1]$ so it follows that $A$ is finite over $B$. This proves " $\Rightarrow$."

Now we prove the converse, assuming $\mathfrak{f}$ to be flat. Working locally on $\mathfrak{X}$ and $\mathfrak{Y}$, we can assume both of these formal schemes are quasi-compact. Thus, $\mathfrak{f}$ can 
be topologically identified with a map between $\widetilde{k}$-schemes of finite type, and so by $\left[\mathrm{EGA}, \mathrm{IV}_{3}, 9.9 .5\right]$ the non-empty fibers of $\mathfrak{f}$ have dimension $\leq n$ if and only if this is true for the non-empty fibers of $\mathfrak{f}$ over closed points. By Lemma A.1.5, we reduce to the case $\mathfrak{Y}=\operatorname{Spf}(B)$ for a domain $B$ that is finite flat over $R$. The assertion in question is insensitive to composing $\mathfrak{f}$ with the finite flat map $\operatorname{Spf}(B) \rightarrow \operatorname{Spf}(R)$. Since $\mathfrak{f}$ is flat, so any non-empty open in $\mathfrak{X}$ gives rise to a non-empty open in $X$ under Raynaud's functor, we therefore just need to prove

$$
\operatorname{dim}\left(A \otimes_{R} k\right)=\operatorname{dim}\left(A / \mathfrak{m}_{R} A\right)
$$

for a flat topologically finitely presented $R$-algebra $A$.

The inequality $\leq$ in (A.2.2) was proved above, even without $R$-flatness conditions on $A$. For the reverse inequality, we need $R$-flatness. Let $d=\operatorname{dim}\left(A \otimes_{R} k\right)$. We will construct a finite $R$-algebra map $R\left\langle\left\langle t_{1}, \ldots, t_{d}\right\rangle\right\rangle \rightarrow A$, from which it follows that $A / \mathfrak{m}_{R} A$ is finite over a $d$-variable polynomial ring over the residue field of $R$ and hence has dimension at most $d$. To construct such a finite $R$-algebra map, choose a surjection

$$
R\left\langle\left\langle t_{1}, \ldots, t_{n}\right\rangle\right\rangle \rightarrow A .
$$

Since the change of variables in [BGR, 5.2.4/1] is of the form $X_{i} \mapsto X_{i}+X_{n}^{c_{i}}$, it follows from the proof of Noether normalization for affinoid algebras in [BGR, $6.1 .2 / 1]$ that we may make a change of variables over $R$ so that $R\left\langle\left\langle t_{1}, \ldots, t_{d^{\prime}}\right\rangle\right\rangle$ injects into $A \subseteq A \otimes_{R} k$ for some $d^{\prime} \geq 0$ (the $R$-flatness ensures that $A$ injects into $A \otimes_{R} k$ ) such that this injection into $A$ is a finite ring map. Applying the functor $(\cdot) \otimes_{R} k$, we deduce that $A \otimes_{R} k$ is a finite extension ring of a Tate algebra in $d^{\prime}$ variables, so $d^{\prime}=d$. This completes the proof of (A.2.2), and so proves (1).

Now consider (2). It is obvious that $f$ must be flat, and Lemma A.1.5 provides the surjectivity (since $\mathfrak{f}$ is flat). We may also use Lemma A.1.5 to reduce the fibral properties of geometric reducedness and equidimensionality of dimension $d$ to the special case when $\mathfrak{Y}=\operatorname{Spf} R$ and $\mathfrak{X}=\operatorname{Spf} A$ is affine. By hypothesis, $A / \mathfrak{m}_{R} A$ is geometrically reduced over the residue field $R / \mathfrak{m}_{R}$ and it has pure dimension $d$. We need to prove that $\operatorname{Sp}\left(k \otimes_{R} A\right)$ is geometrically reduced over $k$ and has pure dimension $d$. Since geometric reducedness is a property that may be checked over a single perfect extension of $k$ and the initial assumptions on $A$ are preserved under arbitrary analytic extension on $k$, we can assume $k$ is algebraically closed. More specifically, allowing $k$ to be arbitrary, it suffices to prove that $k \otimes_{R} A$ is reduced and has pure dimension $d$ if $A / \mathfrak{m}_{R} A$ is reduced with pure dimension $d$.

To prove that $k \otimes_{R} A$ is reduced, it is enough to show that $A$ is reduced. If $a \in A$ is nilpotent then the reduction of $a$ into $A / \mathfrak{m}_{R} A$ vanishes, so $a=c a^{\prime}$ for $a^{\prime} \in A$ and $c \in R$ with $|c|<1$. Since $A$ is $R$-flat, clearly $a^{\prime} \neq 0$ if both $c$ and $a$ are nonzero, and also $a^{\prime}$ is nilpotent if $c \neq 0$. Thus, to prove $a=0$ it suffices to prove that for any nonzero $\alpha \in A$ there exists $c \in R-\{0\}$ such that $\alpha=c \alpha^{\prime}$ 
with $\alpha^{\prime} \in A$ and $\alpha^{\prime} \notin \mathfrak{m}_{R} A$. This general claim will also be used in our proof that $k \otimes_{R} A$ has pure dimension $d$, so we record it as a separate lemma:

Lemma A.2.2. For any topologically finitely presented and flat $R$-algebra $A$ and any non-zero $a \in A \otimes_{R} k$, there is some $k^{\times}$-multiple of a that is in $A$ but is not in $\mathfrak{m}_{R} A$.

I am grateful to L. Lipshitz for the following proof that is much simpler than my original argument.

Proof. We can write $A=R\left\langle\left\langle t_{1}, \ldots, t_{n}\right\rangle\right\rangle / I$ with $I=\left(f_{1}, \ldots, f_{m}\right)$ a finitely generated ideal, and we may suppose that $a \in A$. Let $I^{\prime}$ denote the ideal generated by the $f_{j}$ 's in the Tate algebra $k\left\langle\left\langle t_{1}, \ldots, t_{n}\right\rangle\right\rangle$. Since $A$ is $R$-flat, it follows that $I=I^{\prime} \cap R\left\langle\left\langle t_{1}, \ldots, t_{n}\right\rangle\right\rangle$. Let $F \in R\left\langle\left\langle t_{1}, \ldots, t_{n}\right\rangle\right\rangle$ be a representative for our nonzero $a$, so $F \notin I^{\prime}$. Working in the $n$-variable Tate algebra over $k$, viewed as a Banach algebra with respect to the usual sup norm, it follows from the closedness of ideals that

$$
\inf _{h \in I^{\prime}}\|F-h\|>0 .
$$

By [BGR, 5.2.7/8], this infimum is attained and has the form $|c|$ for some $c \in k$. Since $|c| \leq\|F\| \leq 1$ and $|c|=\left\|F-h_{0}\right\|$ for some $h_{0} \in I^{\prime}$, we deduce that $\left\|h_{0}\right\| \leq 1$. In particular, $h_{0} \in I^{\prime} \cap R\left\langle\left\langle t_{1}, \ldots, t_{n}\right\rangle\right\rangle=I$. Replacing $F$ with $F-h_{0}$ reduces us to the case $|c|=\|F\|$. Thus, $F / c$ has integral coefficients, so $a / c \in A$. Replacing $a$ with $a / c$ reduces us to the case $|c|=1$, so $\|F-h\|=1$ for all $h \in I$. Since such elements $F-h$ constitute exactly the representatives of $a$ relative to the given presentation of $A$, it follows that this $a$ cannot lie in $\mathfrak{m}_{R} A$.

The preceding lemma settles the (geometric) reducedness aspect, and we now turn to the proof that $k \otimes_{R} A$ has pure dimension $d$. We know by (1) that $\operatorname{dim} \operatorname{Sp}\left(k \otimes_{R} A\right) \leq d$ since $\mathfrak{f}: \operatorname{Spf} A \rightarrow \operatorname{Spf} R$ is assumed to have fibers with pure dimension $d$. The problem is therefore one of giving lower bounds to dimensions of irreducible components. If $A$ is a domain, then so is $A \otimes_{R} k$. In this case, all maximal ideals of the $k$-affinoid $A \otimes_{R} k$ have the same height [C1, 2.1.5], so (A.2.2) proves what we need when $A$ is a domain.

More generally, let $\mathfrak{p}$ be a minimal prime of $A$. We claim that $\mathfrak{p}$ cannot contain an ideal of definition of $R$ (that is, $\mathfrak{p} \cap \mathfrak{m}_{R}=(0)$ ), and hence the set of minimal primes of the possibly non-noetherian $A$ is naturally in bijection with the finite set of minimal primes of the noetherian ring $A \otimes_{R} k$. Suppose otherwise, so there exists some non-zero $\pi \in \mathfrak{m}_{R} \cap \mathfrak{p}$. All elements in the maximal ideal $\mathfrak{p} A_{\mathfrak{p}}$ of the local ring $A_{\mathfrak{p}}$ are nilpotent. Since $R$-flatness of $A$ implies that $\pi \in \mathfrak{p} A_{\mathfrak{p}}$ is not a zero divisor, we have a contradiction. By [BL1, 1.1(c)], it follows that $\mathfrak{p}$ is finitely generated, so $A / \mathfrak{p}$ is an $R$-flat topologically finitely presented $R$-algebra. 
Applying (A.2.2) to the $R$-flat domain $A / \mathfrak{p}$, the quotient domain

$$
\left(A \otimes_{R} k\right) /\left(\mathfrak{p} \otimes_{R} k\right) \simeq(A / \mathfrak{p}) \otimes_{R} k
$$

has dimension equal to that of $A /\left(\mathfrak{m}_{R} A+\mathfrak{p}\right)$. Thus, it suffices to check that $\operatorname{dim}\left(A /\left(\mathfrak{m}_{R} A+\mathfrak{p}\right)\right)=d$, and we have already proved the inequality $\leq$ holds.

Let $\overline{\mathfrak{q}}_{1}, \ldots, \overline{\mathfrak{q}}_{m}$ be the finite set of minimal primes of $A / \mathfrak{m}_{R} A$, and let $\mathfrak{q}_{j}$ be the preimage of $\overline{\mathfrak{q}}_{j}$ under $A \rightarrow A / \mathfrak{m}_{R} A$. We claim that $\mathfrak{p}$ lies in some $\mathfrak{q}_{j}$. If this is shown then $A /\left(\mathfrak{m}_{R} A+\mathfrak{p}\right)$ has a $d$-dimensional quotient $A /\left(\mathfrak{m}_{R} A+\mathfrak{q}_{j}\right)=$ $\left(A / \mathfrak{m}_{R} A\right) / \overline{\mathfrak{q}}_{j}$, so by $\left(\right.$ A.2.2) we get $\operatorname{dim}\left(A /\left(\mathfrak{m}_{R} A+\mathfrak{p}\right)\right) \geq d$, giving the desired reverse inequality. To find a $\mathfrak{q}_{j}$ containing $\mathfrak{p}$, it is equivalent to show

$$
\mathfrak{p} \subseteq \bigcup \mathfrak{q}_{j} .
$$

Since $\mathfrak{m}_{R} \subseteq \mathfrak{q}_{j}$ for all $j$, it is necessary and sufficient to show that the image of $\mathfrak{p}$ in the noetherian ring $A / \mathfrak{m}_{R} A$ lies inside of the union of the minimal primes $\overline{\mathfrak{q}}_{j}$. But $\cup \overline{\mathfrak{q}}_{j}$ is exactly the set of zero divisors in $A / \mathfrak{m}_{R} A$ because $A / \mathfrak{m}_{R} A$ is assumed to be reduced, so it suffices for $\mathfrak{p}$ to map into the annihilator of some non-zero element of $A / \mathfrak{m}_{R} A$.

Since $\mathfrak{p} \otimes_{R} k$ is a minimal prime of the noetherian $\operatorname{ring} A \otimes_{R} k$, there exists a non-zero $a \in A \otimes_{R} k$ such that $\mathfrak{p} \otimes_{R} k$ annhilates $a$. By Lemma A.2.2, there exists a $k^{\times}$-scaling $c a$ of $a$ that lies in $A$ and not in $\mathfrak{m}_{R} A$, so $\mathfrak{p}$ maps into the annihilator of a non-zero element in $A / \mathfrak{m}_{R} A$, thereby completing the proof.

Corollary A.2.3. Let $\mathfrak{f}: \mathfrak{X} \rightarrow \mathfrak{Y}$ be a topologically flat map between locally topologically finitely presented formal schemes over $\operatorname{Spf} R$. If the non-empty fibers of $\mathfrak{f}$ are geometrically integral then the non-empty fibers of $f=f^{\text {rig }}$ are geometrically integral (with $f$ surjective when $\mathfrak{f}$ is surjective). If in addition $\mathfrak{f}$ is formally smooth, then so is $f$.

Proof. Since Raynaud's functor is well-behaved with respect to the formation of the sheaf of relative 1-forms, the smoothness aspect follows from the rest. By Theorem A.2.1(2), the only issue that we need to address is the geometric irreducibility of the non-empty fibers of $f$, and since $\mathfrak{f}$ has open image we may suppose it is surjective. By Lemma A.1.5 we may easily reduce to the case when $k$ is algebraically closed and $\mathfrak{Y}=\operatorname{Spf} R$, so (Theorem A.2.1(2)) $X=\mathfrak{X}^{\text {rig }}$ is geometrically reduced and we just have to show that $X$ is irreducible. The hypothesis on the $R$-flat $\mathfrak{X}$ is that the ordinary scheme $\mathfrak{X} \bmod \mathfrak{m}_{R}$ over the residue field is integral, so this hypothesis is inherited by every non-empty open in $\mathfrak{X}$.

We first claim that $X$ is connected. Let $\left\{\mathfrak{X}_{i}\right\}$ be a covering of $\mathfrak{X}$ by non-empty open affines, so each $X_{i}=\mathfrak{X}_{i}^{\text {rig }}$ is a non-empty open affinoid in $X$. Since each open overlap $\mathfrak{X}_{i} \cap \mathfrak{X}_{j}$ is non-empty and $R$-flat, each overlap $X_{i} \cap X_{j}$ is non-empty. Thus, connectivity of $X$ is reduced to that of each $X_{i}$. Hence, by renaming $\mathfrak{X}_{i}$ as $\mathfrak{X}$ we may assume that $\mathfrak{X}=\operatorname{Spf} A$ is affine. Let $e \in \mathscr{O}_{X}(X)=k \otimes_{R} A$ be a nonzero 
idempotent. We want to prove $e=1$. By Lemma A.2.2, if $e \notin A$ then there exists a nonzero $\pi \in \mathfrak{m}_{R}$ such that $e^{\prime}=\pi e \in A$ with $e^{\prime} \notin \mathfrak{m}_{R} A$. However, $e^{\prime 2}=\pi e^{\prime}$ and $A / \mathfrak{m}_{R} A$ is reduced, so we have a contradiction. Thus, $e \in A$. Since $\operatorname{Spec}(A / \pi A)$ is irreducible for every nonzero $\pi \in \mathfrak{m}_{R}$, we must have $e \bmod \pi A \in\{0,1\}$ for all $\pi$. The non-vanishing of $e$ therefore forces $e=1$. Hence, in our initial setup $X$ is indeed connected.

Since $X$ is connected (and non-empty), it follows from the global irreducible decomposition of rigid spaces that if $X$ is not irreducible then there exists $x \in X$ that lies on two distinct irreducible components of $X$. Letting $\mathfrak{U}$ be an open affine in $\mathfrak{X}$ such that the affinoid open $U \stackrel{\text { def }}{=} \mathfrak{U}^{\text {rig }} \subseteq X$ contains $x$, it follows from the relationship between global and local irreducible components (see [C1, 2.2.9]) that $x$ lies on two distinct irreducible components of $U$. Hence, we may rename $\mathfrak{U}$ as $\mathfrak{X}$ to reduce to the affine case $\mathfrak{X}=\operatorname{Spf} A$. Since $X=\operatorname{Sp}\left(k \otimes_{R} A\right)$ is reduced, irreducibility of $X$ is equivalent to $k \otimes_{R} A$ being a domain. To prove that $X$ is irreducible it is therefore equivalent to prove that $A$ is a domain. Choose nonzero $a, a^{\prime} \in A$. By Lemma A.2.2 there exist non-zero $c, c^{\prime} \in R$ such that $a / c, a^{\prime} / c^{\prime} \in A-\mathfrak{m}_{R} A$. For the purposes of proving $a a^{\prime} \neq 0$, we may replace $a$ and $a^{\prime}$ with $a / c$ and $a^{\prime} / c^{\prime}$ respectively to get to the case when $a$ and $a^{\prime}$ have nonzero images $\bar{a}$ and $\bar{a}^{\prime}$ in $A / \mathfrak{m}_{R} A$. The assumptions on $\mathfrak{X}$ imply that $A / \mathfrak{m}_{R} A$ is a domain, so $\bar{a} \cdot \bar{a}^{\prime} \neq 0$. Hence, $a a^{\prime} \neq 0$.

Theorem A.2.4. Let $f: X \rightarrow Y$ be a quasi-separated map of rigid spaces, with $Y$ quasi-separated. Let $k^{\prime} / k$ be an analytic extension field. Let $f^{\prime}: X^{\prime} \rightarrow Y^{\prime}$ be the morphism of rigid spaces over $k^{\prime}$ induced by extension of scalars. Let $\mathbf{P}$ denote one of the following properties of a morphism of rigid spaces:

(a) flat,

(b) all fibers have dimension $\leq n$,

(c) each non-empty fiber is equidimensional with dimension n,

(d) étale,

(e) smooth,

(f) closed immersion,

(g) monomorphism,

(h) separated,

(i) isomorphism,

(j) quasi-compact open immersion,

(k) quasi-compact,

(l) finite,

(m) proper,

(n) faithfully flat and quasi-compact,

(o) flat with geometrically reduced fibers,

(p) flat and quasi-compact with geometrically reduced and geometrically connected (possibly empty) fibers. 
Then $f$ satisfies the property $\mathbf{P}$ if and only if $f^{\prime}$ does.

The analogue of Theorem A.2.4 with respect to fpqc base change is given in [C3, Thm. 4.2.7], where the analogue of (j) does not require the quasi-compactness restriction.

Proof. For (a), (b), and (c), we may assume $X$ and $Y$ are affinoid, say $X=\operatorname{Sp}(A)$, $Y=\operatorname{Sp}(B)$. By $[\mathrm{C} 1,1.1 .5(1)]$, the natural map $A \rightarrow A^{\prime}=k^{\prime} \widehat{\otimes}_{k} A$ is faithfully flat, and likewise for $B \rightarrow B^{\prime}$. If $f^{\prime}$ is flat then it follows formally that $f$ must be flat. Thus, (a) descends. Noether normalization ensures that for any extension $K^{\prime} / K$ of non-archimedean fields we have $\operatorname{dim}(C)=\operatorname{dim}\left(K^{\prime} \widehat{\otimes}_{K} C\right)$ for any $K$ affinoid algebra $C$. Related elementary methods (see $[\mathrm{C} 1,2.1 .5]$ ) show that $C$ is equidimensional with dimension $d$ if and only if $K^{\prime} \widehat{\otimes}_{K} C$ is equidimensional with dimension $d$. Thus, (b) and (c) descend (since every fiber of $f$ becomes a fiber of $f^{\prime}$ upon suitable extension of the base field of the fiber).

To go in reverse and deduce flatness and fiber-dimension conditions for $f^{\prime}$ from the analogous property for $f$, we will use Raynaud's theory of formal models for (a) and (b), and we will use Berkovich spaces for (c). Since $f$ has quasi-compact and quasi-separated (even affinoid) source and target, if $f$ is flat (resp. has fiber dimension $\leq n$ ) then by $\left[\right.$ BL2, 5.10] we have $f=\mathfrak{f}^{\text {rig }}$ for some morphism $\mathfrak{f}$ between quasi-compact flat formal models, with $\mathfrak{f}$ flat (resp. having fiber dimension $\leq n$ ). Thus, the base change $\mathfrak{f}^{\prime}$ is flat (resp. has fiber dimension $\leq n$ ). Hence, $f^{\prime}=\mathfrak{f}^{\text {rig }}$ is flat (resp. has fiber dimension $\leq n$, by Theorem A.2.1(1)). This settles (a) and (b).

To handle preservation of (c) under extension of the base field, recall Kiehl's theorem [K, 3.7] that for any map $f: X \rightarrow Y$ between rigid-analytic spaces, the loci $F_{m}(f)=\left\{x \in X \mid \operatorname{dim}_{x} f^{-1}(f(x)) \geq m\right\}$ are Zariski-closed in $X$. Since we assume that all non-empty fibers of $f$ are equidimensional of dimension $n$, we conclude that $F_{n}(f)=X$ and (by (b)) all fibers of $f^{\prime}$ have dimension $\leq n$. Our problem is therefore to prove that $F_{n}\left(f^{\prime}\right)=X^{\prime}$, so it suffices to prove:

Lemma A.2.5. For an arbitrary map $f: X \rightarrow Y$ between quasi-separated rigid spaces over $k$ and for an arbitrary integer $m$, the analytic sets $k^{\prime} \widehat{\otimes}_{k} F_{m}(f)$ and $F_{m}\left(f^{\prime}\right)$ in $X^{\prime}$ coincide.

Proof. We may (and do) assume $X=\mathrm{Sp}(A)$ and $Y=\operatorname{Sp}(B)$. Kiehl's method of proof of analyticity of the loci $F_{m}(f)$ is given in terms of commutative-algebra constructions whose interaction with analytic change in the base field is rather difficult to understand. Thus, to relate $F_{m}(f)$ and $F_{m}\left(f^{\prime}\right)$ we use a different point of view: Berkovich spaces. 
Let $\mathscr{M}(A)$ and $\mathscr{M}(B)$ be the strictly $k$-affinoid Berkovich spaces associated to $A$ and $B$, and let

$$
\varphi: \mathscr{M}(A) \rightarrow \mathscr{M}(B)
$$

be the $k$-morphism corresponding to $f: \operatorname{Sp}(A) \rightarrow \operatorname{Sp}(B)$. Finally, write $A^{\prime}, f^{\prime}$, etc. to denote the base change over $k^{\prime}$. For any point $y \in \mathscr{M}(B)$ with associated completed residue field $k(y)$, the fiber $\varphi^{-1}(y)$ in the sense of Berkovich is the strictly $k(y)$-affinoid space

$$
\varphi^{-1}(y)=\mathscr{M}\left(k(y) \widehat{\otimes}_{B} A\right)=\mathscr{M}\left(k(y) \widehat{\otimes}_{k(y) \widehat{\otimes}_{k} B}\left(k(y) \widehat{\otimes}_{k} A\right)\right),
$$

where the map $k(y) \widehat{\otimes}_{k} B \rightarrow k(y)$ is the rational point defined by the continuous $k$-algebra map $y: B \rightarrow k(y)$.

Let us now briefly digress to discuss pointwise dimension on certain Berkovich spaces. In general, for any non-archimedean field $K$, strictly $K$-affinoid algebra $C$, and point $x \in \mathscr{M}(C)$, we define $\operatorname{dim}_{x} \mathscr{M}(C)$ to be the maximum of the dimensions $\operatorname{dim} C / \mathfrak{p}$ for minimal primes $\mathfrak{p}$ of $C$ such that the subset $\mathscr{M}(C / \mathfrak{p}) \subseteq \mathscr{M}(C)$ contains the point $x$. This notion of pointwise dimension is well-behaved with respect to change in the ground field in the sense that if $K^{\prime} / K$ is an analytic extension field and $C^{\prime}=K^{\prime} \widehat{\otimes}_{K} C$ then for any $x^{\prime} \in \mathscr{M}\left(C^{\prime}\right)$ lying over $x$ under the canonical map $\mathscr{M}\left(C^{\prime}\right) \rightarrow \mathscr{M}(C)$, we have

$$
\operatorname{dim}_{x^{\prime}} \mathscr{M}\left(C^{\prime}\right)=\operatorname{dim}_{x} \mathscr{M}(C) .
$$

Indeed, in view of the definition, it suffices to to recall the elementary fact [C1, 2.1.5] that if $C$ is a strictly $K$-affinoid domain then the strictly $K^{\prime}$-affinoid algebra $K^{\prime} \widehat{\otimes}_{K} C$ is equidimensional of dimension $\operatorname{dim} C$.

In [Ber2, 1.1.6], Berkovich adapts Kiehl's methods to Berkovich spaces, and he thereby proves that the loci $F_{m}(\varphi)=\left\{x \in \mathscr{M}(A) \mid \operatorname{dim}_{x} \varphi^{-1}(\varphi(x)) \geq m\right\}$ are analytic sets in $\mathscr{M}(A)$. Since $F_{m}(\varphi)$ is an analytic set, its preimage under the morphism of Berkovich spaces

$$
k^{\prime} \widehat{\otimes}_{k} \mathscr{M}(A) \simeq \mathscr{M}\left(A^{\prime}\right) \rightarrow \mathscr{M}(A)
$$

is the analytic set $k^{\prime} \widehat{\otimes}_{k} F_{m}(\varphi)$. For any analytic set $Z \subseteq \mathscr{M}(A), k^{\prime} \widehat{\otimes}_{k} Z \subseteq$ $\mathscr{M}\left(A^{\prime}\right)$ meets the set of "classical points" $\operatorname{Sp}\left(A^{\prime}\right) \subseteq \mathscr{M}\left(A^{\prime}\right)$ in exactly the locus $k^{\prime} \widehat{\otimes}_{k}(\operatorname{Sp}(A) \cap Z)$. Since $(\operatorname{Sp} A) \cap F_{m}(\varphi)=F_{m}(f)$, we conclude that the preimage of $F_{m}(\varphi)$ in $\mathscr{M}\left(A^{\prime}\right)$ meets $\operatorname{Sp} A^{\prime}$ in $k^{\prime} \widehat{\otimes}_{k} F_{m}(f)$. The equality $\left(\operatorname{Sp} A^{\prime}\right) \cap$ $F_{m}\left(\varphi^{\prime}\right)=F_{m}\left(f^{\prime}\right)$ therefore reduces the desired equality $k \widehat{\otimes}_{k} F_{m}(f)=F_{m}\left(f^{\prime}\right)$ inside $\operatorname{Sp} A^{\prime}$ to the assertion that the preimage of $F_{m}(\varphi)$ in $\mathscr{M}\left(A^{\prime}\right)$ is $F_{m}\left(\varphi^{\prime}\right)$. For $x^{\prime} \in \mathscr{M}\left(A^{\prime}\right)$ over $x \in \mathscr{M}(A)$, we have $\varphi^{\prime-1}\left(y^{\prime}\right)=k\left(y^{\prime}\right) \widehat{\otimes}_{k(y)} \varphi^{-1}(y)$ for the point $y^{\prime}=\varphi^{\prime}\left(x^{\prime}\right)$ over the point $y=\varphi(x)$, so by (A.2.3) it follows that $\operatorname{dim}_{x^{\prime}} \varphi^{\prime-1}\left(\varphi^{\prime}(y)\right)=\operatorname{dim}_{x} \varphi^{-1}(\varphi(x))$ for $x=\varphi\left(x^{\prime}\right)$. Hence, $F_{m}\left(\varphi^{\prime}\right)$ is indeed the preimage of $F_{m}(\varphi)$. 
Turning to (d), since étale maps are flat maps whose $\Omega^{1}$ vanishes, we deduce (d) from (a). For (e), we first note that, as in algebraic geometry, a smooth morphism can be described locally (for the Tate topology) as an étale morphism to a relative ball. Such a description is preserved under extension of the ground field, by (d), so smoothness is preserved under extension of the ground field. To go in the reverse direction and descend smoothness, suppose $f^{\prime}$ is smooth. Thus, $f^{\prime}$ is flat and $\Omega_{X^{\prime} / Y^{\prime}}^{1}$ is locally free, so by (a) we conclude that $f$ is flat and $\Omega_{X / Y}^{1}$ is locally free. By working locally on $X$, we may assume that $\Omega_{X / Y}^{1}$ has constant rank $d$, and hence the same holds for $\Omega_{X^{\prime} / Y^{\prime}}^{1}$. The map $f^{\prime}$ must therefore have all fibers with pure dimension $d$, so the same holds for $f$ (by the trivial direction in (c)). The flatness of $f$ reduces the smoothness of $f$ to a fibral problem, and so it remains to check that a pure $d$-dimensional rigid space $Z$ over a non-archimedean field $k$ is $k$-smooth if $\Omega_{Z / k}^{1}$ is locally free of rank $d$. Smoothness is equivalent to "geometric regularity", and so since the property of having pure dimension $d$ is preserved by change of the base field [C1, 2.1.5], we may assume $k$ is algebraically closed. In this case all points are $k$-rational and hence we can deduce regularity from the properties of $\Omega_{Z / k}^{1}$ as in the algebraic case (using that a $k$-linear derivation $A \rightarrow M$ from a $k$-affinoid to a finite $A$-module is automatically continuous [BKKN, 2.1.5]). This finishes (e).

Cases $(\mathrm{k})-(\mathrm{p})$ will be treated shortly, but let us see how to settle everything else if we grant $(\mathrm{k})-(\mathrm{p})$. It is obvious that (f) reduces to (l). Since $f$ is a monomorphism (resp. separated) if and only if $\Delta_{f}$ is an isomorphism (resp. a closed immersion), (g) and (h) may also be reduced to (l) (here we use the faithful flatness of $A \rightarrow A^{\prime} \stackrel{\text { def }}{=} k^{\prime} \widehat{\otimes}_{k} A$ and the well-known canonical isomorphism $k^{\prime} \widehat{\otimes}_{k} M \simeq A^{\prime} \otimes_{A} M$ for finite $A$-modules, as in [C1, 1.1.5(1)]). Clearly (i) reduces to (f) and (n). By [BL2, 5.4(a)], quasi-compact open immersions are the same as quasi-compact flat monomorphisms. Thus, (j) follows from (a), (g), and (k).

It remains to consider the properties $(\mathrm{k})-(\mathrm{p})$. For $(\mathrm{k}),(\mathrm{l})$, and $(\mathrm{m})$, the preservation of the property under passage from $f$ to $f^{\prime}$ is trivial. We now consider the problem of descending such properties of $f^{\prime}$ to properties of $f$. Due to the quasicompactness condition in Raynaud's theory of formal models, we begin with (k): assume $f^{\prime}$ is quasi-compact. In order to prove that $f$ is quasi-compact, we may assume $Y$ is affinoid. Let $\left\{U_{i}\right\}$ be an admissible open affinoid covering of $X$, so $\left\{U_{i}^{\prime}\right\}$ is an admissible open affinoid covering of $X^{\prime}$. By quasi-compactness of $f^{\prime}$, a finite subcollection $\left\{U_{i_{1}}^{\prime}, \ldots, U_{i_{n}}^{\prime}\right\}$ is an admissible covering of $X^{\prime}$. Since $X$ is quasi-separated, the union $U=\cup U_{i_{j}} \subseteq X$ is an admissible open with the $U_{i_{j}}$ 's an admissible covering of $U$. It remains to check that $U=X$. If not, there is a morphism $\operatorname{Sp}\left(k_{1}\right) \rightarrow X$ with $k_{1} / k$ a finite extension and $\operatorname{Sp}\left(k_{1}\right) \times_{X} U=\emptyset$, so $\operatorname{Sp}\left(k_{1}\right) \times_{X} U_{i_{j}}=\emptyset$ for all $j$. Applying the base change functor $k^{\prime} \widehat{\otimes}_{k}(\cdot)$, the map $\operatorname{Sp}\left(k^{\prime} \otimes_{k} k_{1}\right) \rightarrow X^{\prime}$ has empty fiber over all $U_{i_{j}}^{\prime}$, an absurdity. This settles (k). 
To descend finiteness and thereby settle (l), suppose $f^{\prime}: X^{\prime} \rightarrow Y^{\prime}$ is finite. By (b) and (k), $f: X \rightarrow Y$ is quasi-compact and quasi-separated with finite fibers. We can assume $Y$ is affinoid, so both $X$ and $Y$ are quasi-compact and quasiseparated rigid spaces. By [BL1, 5.10], there exists a quasi-finite formal model $\mathfrak{f}: \mathfrak{X} \rightarrow \mathfrak{Y}$ for $f$, so $\mathfrak{f}^{\prime}$ is a formal model for the proper $f^{\prime}$. Since $f^{\prime}$ is proper, $\mathfrak{f}^{\prime}$ must be proper [L, 2.5, 2.6]. Hence, ordinary fpqc descent for schemes (over the residue fields) implies that $\mathfrak{f}$ is proper. By properness and quasi-finiteness of $\mathfrak{f}$, it follows (working modulo ideals of definition of $R$ ) that $\mathfrak{f}$ is a finite map of formal models and hence $f=f^{\text {rig }}$ is a finite map.

Now assume that $f^{\prime}$ is proper. By (h) we know that $f$ is quasi-compact and quasi-separated, and we must prove that $f$ is proper. By working locally we may assume that $Y$ is quasi-compact and quasi-separated (even affinoid), whence the same holds for $X$. To prove that $f$ is proper, we again use the theory of formal models. Let $\mathfrak{f}$ be a formal model of $f$. The base change $\mathfrak{f}^{\prime}$ is a formal model of the proper $f^{\prime}$, so $\mathfrak{f}^{\prime}$ is proper. As in the proof of (l), it follows that $\mathfrak{f}$ must be proper. By [Te, Cor. 4.4, Cor. 4.5], $f=f^{\text {rig }}$ is therefore proper. This settles (m).

Consider (n). From what has already been proved, we may assume $f$ and $f^{\prime}$ are both quasi-compact and flat, and we must show that $f$ is surjective if and only if $f^{\prime}$ is surjective. We may work locally on $Y$, so we may assume $X$ and $Y$ are quasi-compact (and quasi-separated, by taking $Y$ to be affinoid and then replacing $X$ with a finite disjoint union of affinoids). Using the theory of formal models, we just have to check that if $\mathfrak{f}$ is a flat formal model of $f$, then $f$ is surjective if and only if $\mathfrak{f}$ is surjective. Since $\mathfrak{f}$ is surjective if and only if it is surjective on closed points, it follows from Lemma A.1.5 that surjectivity of $f$ forces surjectivity of $\mathfrak{f}$. Conversely, suppose that the flat $\mathfrak{f}$ is surjective. To prove that $f$ is surjective, we may first reduce to the case where $\mathfrak{X}=\operatorname{Spf}(A)$ and $\mathfrak{Y}=\operatorname{Spf}(B)$ are affine. The flat map of topological $R$-algebras $B \rightarrow A$ is faithfully flat (as this can be checked by working with localizations at maximal ideals, all of which contain $\mathfrak{m}_{R}$ ), so $k \otimes_{R} B \rightarrow k \otimes_{R} A$ is faithfully flat. Thus, $f=\mathfrak{f}^{\text {rig }}=k \otimes_{R} \mathfrak{f}$ is surjective.

Next, we address (o). Descent from $f^{\prime}$ to $f$ is obvious, and for the converse we may work locally on both $X$ and $Y$. Thus, we may suppose that $X$ and $Y$ are quasi-compact and quasi-separated. It is permissible to also replace $Y$ with a quasi-compact (and quasi-separated) étale cover. By the Reduced Fiber Theorem [BLR2], since $f$ is flat with geometrically reduced fibers we can make a base change by a suitable quasi-compact étale cover of $Y$ in order to arrange that $f=\mathfrak{f}^{\text {rig }}$ for a flat map $\mathfrak{f}: \mathfrak{X} \rightarrow \mathfrak{Y}$ between $R$-flat quasi-compact formal models such that the fibers of $\mathfrak{f}$ (as ordinary schemes over residue fields on $\mathfrak{Y}$ ) are geometrically reduced. Using base change by $\operatorname{Spf} R^{\prime} \rightarrow \operatorname{Spf} R$, the map $\mathfrak{f}^{\prime}: \mathfrak{X}^{\prime} \rightarrow \mathfrak{Y}^{\prime}$ is also flat with geometrically reduced fibers. The generic fiber $f^{\prime}$ of $\mathfrak{f}^{\prime}$ is therefore flat and, 
by the methods in the proof of Theorem A.2.1, it must have have geometrically reduced fibers.

Finally, consider (p). As with (o), descent from $f^{\prime}$ to $f$ is trivial. For the converse, we may assume that $Y$ is affinoid, so $X$ is quasi-compact and quasiseparated. We may suppose that $Y$ is affinoid, say with a formal model $\mathfrak{Y}$. By suitable admissible blow-up on $\mathfrak{Y}$ we may arrange that there exists a flat formal model $\mathfrak{f}: \mathfrak{X} \rightarrow \mathfrak{Y}$ for $f$ with geometrically reduced fibers, and by passing to the projection $p_{1}: \mathfrak{X} \times_{\mathfrak{Y}} \mathfrak{X} \rightarrow \mathfrak{X}$ in the role of $\mathfrak{f}$ we may assume that there exists a section $\mathfrak{s}$. By [C1, 3.2.1], a connected quasi-separated rigid space with a rational point is geometrically connected. Thus, we need only show that $f^{\prime}$ has connected fibers.

Every point of $\mathfrak{Y}$ is the underlying point of a morphism $\mathfrak{y}: \operatorname{Spf} B \rightarrow \mathfrak{Y}$ with $B$ a local domain that is finite and flat over $R$ (Lemma A.1.5), and the base change $\mathfrak{X}_{\mathfrak{y}}$ is a flat and topologically finitely presented formal scheme over $\operatorname{Spf} B$ with generic fiber $X_{y}$ that is connected. Hence, $\mathfrak{X}_{\mathfrak{y}}$ cannot have non-trivial idempotents, and so it is connected. Thus, all fibers of $\mathfrak{X} \rightarrow \mathfrak{Y}$ over closed points are connected, and so the section $\mathfrak{s}$ ensures that all such fibers are geometrically connected. By [EGA, $\left.\mathrm{IV}_{3}, 9.7 .9\right]$, all fibers of $\mathfrak{X} \rightarrow \mathfrak{Y}$ are therefore geometrically connected. The same holds after the base change by $\operatorname{Spf}\left(R^{\prime}\right) \rightarrow \operatorname{Spf}(R)$, so to prove connectivity of the fibers of $f^{\prime}$ we are reduced to checking that if $B^{\prime}$ is a local finite flat domain over $R^{\prime}$ and $\mathfrak{X}^{\prime}$ is a connected formal scheme that is flat and topologically finitely presented over Spf $B^{\prime}$ with reduced fiber modulo $\mathfrak{m}_{B^{\prime}}$ then its generic fiber $X^{\prime}$ is connected. We may certainly suppose $B^{\prime}=R^{\prime}$, and then the connectivity is proved by arguing as in the proof of Corollary A.2.3 (via passage to the affine case and using Lemma A.2.2).

One further important property of flatness is the fibral criterion for a map to be flat or an isomorphism:

Corollary A.2.6. Let $X$ and $Y$ be rigid spaces over $S$, and $f: X \rightarrow Y$ an S-morphism.

(1) If $X$ and $Y$ are $S$-flat and $f_{s}: X_{s} \rightarrow Y_{s}$ is flat for all $s \in S$ then $f$ is flat. If moreover $f$ is quasi-compact then $f_{s}$ is an isomorphism for all $s \in S$ if and only if $f$ is an isomorphism. In both cases, it suffices to work with geometric fibers over $S$ when $X$ and $Y$ are quasi-separated over $S$.

(2) If $f$ induces a flat map on all infinitesimal fibers over $S$, then $f$ is flat. The same conclusion holds for the property of being an isomorphism if $f$ is quasi-compact. In both cases it suffices to work with infinitesimal geometric fibers over $S$ if $X$ and $Y$ are quasi-separated over $S$.

(3) Two $S$-maps $f, g: X \rightrightarrows Y$ that coincide on all infinitesimal fibers over $S$ are equal. It suffices to work with infinitesimal geometric fibers over $S$ if $X$ and $Y$ are quasi-separated over $S$. 
Remark A.2.7. The assertions concerning isomorphisms are false if we drop the quasi-compactness hypothesis. For example, let $Y=S=\operatorname{Sp} k\langle\langle t\rangle$ be the "closed" unit ball and let $X$ be the disjoint union of the "boundary" $\{|t|=1\}$ and the "open" unit ball $\{|t|<1\}$.

Proof. Theorem A.2.4 shows the equivalence of working with geometric and ordinary (infinitesimal) fibers. Thus, it suffices to consider ordinary fibers and infinitesimal fibers. This case follows by standard arguments ( $c f$. the proof of [C3, Thm. A.2.6]), using the local flatness criterion [Mat, 23.3] to handle flatness and the Krull intersection theorem to handle (3). The only delicate point is to show that if $f$ is flat and quasi-compact, then $f$ is an isomorphism if $f_{s}$ is an isomorphism for all $s \in S$. Since $f$ is bijective and quasi-compact, by [BGR, 9.5.3/5] it follows that $f$ is an isomorphism if and only if $f$ is a local isomorphism. It therefore suffices to show that the natural map $\mathscr{O}_{Y, f(x)} \rightarrow \mathscr{O}_{X, x}$ is an isomorphism for all $x \in X$. This map of rings is a priori finite and flat (since $f$ is quasi-finite and flat), so it is enough to check surjectivity of the ring map. By Nakayama's Lemma this can be checked modulo $\mathfrak{m}_{s}$, where it follows from the assumption that $f_{s}$ is an isomorphism.

\section{Appendix B. Algebraic theory of generalized elliptid CURVes}

B.1. Deligne-Rapoport semistable curves of genus 1. For the convenience of the reader, we begin by reviewing some basic algebraic notions, but we generally omit proofs.

For any $n \geq 2$, the standard (Néron) $n$-gon $C_{n}$ over a base scheme $S$ is the scheme obtained from $\mathbf{P}_{S}^{1} \times \mathbf{Z} / n \mathbf{Z}$ by gluing the section 0 on $\mathbf{P}_{S}^{1} \times\{i\}$ to the section $\infty$ on $\mathbf{P}_{S}^{1} \times\{i+1\}$ for all $i \in \mathbf{Z} / n \mathbf{Z}$. The formation of this gluing is naturally compatible with base change (for flatness reasons) and it is proper over $S$.

The map " $t \mapsto\left(t^{2}+1, t\left(t^{2}+1\right)\right)$ " from $\mathbf{P}^{1}$ to the nodal plane curve $C_{1}: y^{2} z=$ $x^{3}-x^{2} z$ gives $C_{1}$ the universal property to be the gluing of $\mathbf{P}^{1}$ to itself along the sections 0 and $\infty$. The relative smooth locus $C_{1}^{\mathrm{sm}}$ of $C_{1}$ is canonically identified with $\mathbf{G}_{m}=\mathbf{P}^{1}-\{0, \infty\}$, and the $C_{n}$ 's form a compatible tower of finite étale covers of $C_{1}$. Over a base scheme $S$ we call $C_{1}$ the standard (Néron) 1-gon.

Definition B.1.1. A Deligne-Rapoport $(D R)$ semistable genus-1 curve over a scheme $S$ is a proper, flat, finitely presented map $f: C \rightarrow S$ such that every fiber of $f$ is a geometrically connected and geometrically reduced curve with at worst ordinary double point singularities and with trivial dualizing sheaf.

In [DR] such curves are called stable genus-1 curves; we prefer the terminology in Definition B.1.1 because such marked curves are generally semistable but not 
stable in the sense now commonly used in algebraic geometry. In [DR, II, 1.2, 1.3] it is shown that the DR semistable genus- 1 curves over an algebraically closed field are exactly the smooth genus- 1 curves and the standard $n$-gons $C_{n}$ for $n \geq 1$ (this classification also holds over separably closed fields). $1.2]$.

The following is an analogue of a higher-genus theorem for stable curves [DM,

Theorem B.1.2. Let $f: C \rightarrow S$ be a DR semistable genus-1 curve, and let $D \hookrightarrow C$ be a relative effective Cartier divisor with degree $d \geq 1$ and support in $C^{\mathrm{sm}}$. Assume that $D$ meets all irreducible components of all geometric fibers of $C \rightarrow S$. For all $r \geq 1, \mathscr{O}(r D)$ is $S$-ample and $\mathrm{R}^{1} f_{*}(\mathscr{O}(r D))=0$. Moreover, $f_{*}(\mathscr{O}(r D))$ is locally free of rank $r d$ and its formation is compatible with base change.

The natural map $f^{*} f_{*}(\mathscr{O}(r D)) \rightarrow \mathscr{O}(r D)$ is surjective for $r \geq 3$, so the natural map $C \rightarrow \mathbf{P}\left(f_{*}(\mathscr{O}(r D))\right)$ is a closed immersion for $r \geq 3$.

In the study of families, a basic fact is:

Lemma B.1.3. [DR, II, 1.5] Let $f: C \rightarrow S$ be a proper flat map of finite presentation. The set of $s \in S$ over which the fiber is a DR semistable genus-1 curve is Zariski-open.

Let $C$ be a DR semistable genus- 1 curve and let $D$ be a relative effective Cartier divisor on $C^{\mathrm{sm}}$ that is finite over $S$ and meets every fiber $C_{s}$. By [DR, IV, 1.2] there is a DR semistable genus-1 curve $\bar{C}_{/ S}$ and a proper $S$-morphism

$$
u: C \rightarrow \bar{C}
$$

such that on geometric fibers over $S$ the map $u$ contracts precisely the irreducible components disjoint from $D$. This "contraction of $C$ away from $D$ " is unique up to unique isomorphism (though $C$ can have non-trivial automorphisms over $\bar{C}$ ), so its formation is naturally compatible with base change. Since the open subscheme $\bar{C}^{\mathrm{sm}} \subseteq \bar{C}$ is universally schematically dense over $S$ [EGA, $\left.\mathrm{IV}_{3}, 11.10 .4,11.10 .10\right]$, it follows from the uniqueness that for any $S$-automorphism $\alpha$ of $C$ taking $D$ into $D$ there is a unique $S$-automorphism $\bar{\alpha}$ of $\bar{C}$ such that $\bar{\alpha} \circ u=u \circ \alpha$. In more precise terms, the contraction construction (B.1.1) is functorial with respect to isomorphisms in the pair $(C, D)$.

Remark B.1.4. Since the contraction $\bar{C}$ is constructed as Proj of a suitable finitely generated and graded quasi-coherent sheaf of $\mathscr{O}_{S}$-algebras that is chosen by means of the theory of Hilbert schemes, the rigid-analytic analogue of contractions in Theorem 2.1.6 requires the Proj construction in the rigid-analytic category [C3, $2.3]$. 
Let $f: C \rightarrow S$ be a proper, flat, and finitely presented map whose fibers have pure dimension 1. The first Fitting-ideal sheaf of $\Omega_{C / S}^{1}$ defines a canonical closed subscheme structure on $C^{\text {sing }}=C-C^{\mathrm{sm}}$ whose formation is compatible with base change over $S$. We call $C^{\text {sing }}$ the non-smooth locus on the curve $C$. In case the fibers are semistable curves, one sees by étale-local calculation that this Fitting ideal is the annihilator ideal of $\Omega_{C / S}^{2}$.

Definition B.1.5. Let $f: C \rightarrow S$ be a DR semistable genus-1 curve. The locus of non-smoothness of $f$ is the scheme-theoretic image $S^{\infty, f}$ of the $S$-finite $C^{\text {sing }}$ in $S$.

Example B.1.6. By direct calculation, $S^{\infty, f}=S$ when $f: C \rightarrow S$ is the standard $n$-gon over a scheme $S$ for any $n \geq 1$.

\section{B.2. Generalized elliptic curves over schemes.}

Definition B.2.1. A generalized elliptic curve over a scheme $S$ is a triple $(E,+, e)$ where $E$ is a DR semistable genus-1 curve, $+: E^{\mathrm{sm}} \times_{S} E \rightarrow E$ is an $S$-morphism (with $E^{\mathrm{sm}}$ the relative smooth locus of $E$ over $S$ ), and $e \in E^{\mathrm{sm}}(S)$ is a section such that the following properties hold:

- + restricts to a commutative group scheme structure on $E^{\mathrm{sm}}$ with identity section $e$,

- + is an action of $E^{\mathrm{sm}}$ on $E$ such that on each $n$-gon geometric fiber, the translation action by each rational point in the smooth locus induces a rotation on the graph of irreducible components.

By [EGA, $\left.\mathrm{IV}_{3}, 11.10 .4,11.10 .10\right], C_{n}^{\mathrm{sm}}=\mathbf{G}_{m} \times \mathbf{Z} / n \mathbf{Z}$ is universally schematically dense in the standard $n$-gon $C_{n}$ (relative to $S$ ). Thus, there can be at most one action of the group scheme $C_{n}^{\mathrm{sm}}$ on $C_{n}$ compatible with the group law on $C_{n}^{\mathrm{sm}}$ (and this action must be compatible with base change). Such an action always exists: the compatibility of gluing with respect to flat base change (such as $\left.C_{n}^{\text {sm }} \rightarrow S\right)$ ensures that for all $n \geq 1$ the action $\left(\mathbf{G}_{m} \times \mathbf{Z} / n \mathbf{Z}\right) \times_{S}\left(\mathbf{P}_{S}^{1} \times \mathbf{Z} / n \mathbf{Z}\right) \rightarrow$ $\left(\mathbf{P}_{S}^{1} \times \mathbf{Z} / n \mathbf{Z}\right)$ uniquely descends to a morphism

$$
+: C_{n}^{\mathrm{sm}} \times{ }_{S} C_{n} \rightarrow C_{n}
$$

by universal relative schematic denseness, this is an action extending the $S$-group law on $C_{n}^{\mathrm{sm}}$.

Over an algebraically closed field, up to isomorphism the generalized elliptic curves are exactly the smooth elliptic curves and standard $n$-gons with the structure (B.2.1) [DR, II, 1.15]. Whenever we speak of standard polygons over $S$ as generalized elliptic curves, it is always understood that we use the structure (B.2.1). 
Lemma B.2.2. [DR, II, 1.15] Let $f: E \rightarrow S$ be a generalized elliptic curve. The locus of non-smoothness $S^{\infty, f} \hookrightarrow S$ for $f$ is a locally finite (in $S$ ) disjoint union of open subschemes $S_{n}^{\infty, f} \subseteq S^{\infty, f}$ such that the generalized elliptic curve $E$ is isomorphic to the standard n-gon fppf-locally over $S_{n}^{\infty, f}$.

If $n$ is a unit on $S$ then these fppf coverings of $S_{n}^{\infty, f}$ can be taken to be étale coverings.

Remark B.2.3. The cohomological method in the proof of Theorem B.3.2 below shows that if $k$ is an imperfect field of characteristic $p$ then for any positive $n \in p \mathbf{Z}$ there exists a generalized elliptic over $k$ that becomes a standard $n$-gon over a finite extension of $k$ but not over any finite separable extension of $k$.

The following important fact is implicit (but not stated) in $[\mathrm{DR}]$ and underlies the definition of closed substacks "at infinity" in moduli stacks of generalized elliptic curves.

Theorem B.2.4. Let $f: E \rightarrow S$ be a generalized elliptic curve. The formation of the closed subscheme $S^{\infty, f} \hookrightarrow S$ is compatible with base change on $S$.

Proof. See [C2, Thm. 2.1.12] for a proof, and see [C2, Ex. 2.1.11] for a counterexample if "generalized elliptic curve" is weakened to "DR semistable genus-1 curve."

We conclude our review of the algebraic theory with a discussion of how to construct generalized elliptic curve structures on a DR semistable genus-1 curve $C$ without the explicit use of the non-proper curve $C^{\mathrm{sm}}$.

Consider a proper flat semistable curve $C \rightarrow S$ over a scheme $S$ and let $e \in$ $C^{\mathrm{sm}}(S)$ be a section of the relative smooth locus. Since the geometric fibers are reduced and connected, we have $\mathscr{O}_{S} \simeq f_{*} \mathscr{O}_{C}$ universally and so the functor $\mathrm{Pic}_{C / S}$ classifies line bundles on $C$ rigidified along the section $e$. This is a smooth locally separated algebraic space group locally of finite presentation over $S$, and the union $\mathrm{Pic}_{C / S}^{0}=\mathrm{Pic}_{C / S}^{\tau}$ of the fibral identity components is an open subspace that is a semi-abelian algebraic space and classifies line bundles with degree-0 restriction to each irreducible component of a geometric fiber of $C \rightarrow S$.

Let $E \rightarrow S$ be a generalized elliptic curve over a scheme $S$, and consider the natural action map of algebraic spaces

$$
E^{\mathrm{sm}} \times \mathrm{Pic}_{E / S}^{0} \rightarrow \mathrm{Pic}_{E / S}^{0}
$$

arising from the $E^{\mathrm{sm}}$-action on $E$. By [DR, II, 1.13], this action must be trivial. This motivates the relevance of the following powerful result that provides the key to circumventing problems caused by the non-properness of $E^{\mathrm{sm}}$ in the rigidanalytic case. 
Theorem B.2.5. [DR, II, 3.2] Let $f: C \rightarrow S$ be a DR semistable genus-1 curve over a scheme $S$, and let $e \in C^{\mathrm{sm}}(S)$ be a section of the relative smooth locus. Let $G$ be a commutative flat $S$-group scheme locally of finite presentation, and let $\rho: G \times C \rightarrow C$ be an action of $G$ on $C$. Assume that $G$ acts trivially on the algebraic space $\mathrm{Pic}_{C / S}^{0}$ and that $G(\bar{s})$ acts transitively on the set of irreducible components of $C_{\bar{s}}$ for all geometric points $\bar{s}$ of $S$.

There exists a unique generalized elliptic curve structure on $C$ with identity section e such that each $g \in G(T)$ acts on $C_{/ T}$ via translation by $g(e) \in C^{\mathrm{sm}}(T)$ for all $S$-schemes $T$. Moreover, an automorphism $\alpha$ of $C$ commuting with the $G$-action must be translation by $\alpha(e) \in C^{\mathrm{sm}}(S)$.

The case $G=\mathbf{Z}$ in Theorem B.2.5 will be useful in our study of the rigidanalytic case, but for most purposes the following special case will suffice.

Theorem B.2.6. Let $f: C \rightarrow S$ be a DR semistable genus-1 curve over a scheme $S$, and let $D \hookrightarrow C$ be an $S$-ample relative effective Cartier divisor supported in $C^{\mathrm{sm}}$. Assume that $D$ is endowed with a structure of commutative $S$-group scheme and that there is given an action of $D$ on $C$ that extends the group scheme structure on $D$.

This extends to a generalized elliptic curve structure on $C$ if and only if the natural induced action of $D$ on the semi-abelian algebraic space $\mathrm{Pic}_{C / S}^{0}$ is trivial, in which case such a generalized elliptic curve structure on $C_{/ S}$ is unique. The locus of fibers with trivial action is Zariski-open and Zariski-closed in $S$, and over this locus the action is trivial.

Proof. The $S$-ampleness of $D \subseteq C^{\mathrm{sm}}$ ensures that (on geometric fibers) the action of $D(\bar{s})$ on $C_{\bar{s}}$ is transitive on irreducible components of $C_{\bar{s}}$. Thus, the theorem is essentially a special case of Theorem B.2.5 except for the assertions concerning the locus of fibers with trivial action. These are addressed in [C2, Cor. 2.2.3].

When working with the constant group $G=\mathbf{Z}$ in Theorem B.2.5, the triviality condition for the action on $\mathrm{Pic}_{C / S}^{0}$ may again be checked on fibers, due to:

Lemma B.2.7 (Deligne-Rapoport). Let $A \rightarrow S$ be a semi-abelian algebraic space over a scheme $S$. Let $g$ and $h$ be two endomorphisms of $A_{/ S}$. There exists a unique Zariski-open and Zariski-closed $U \subseteq S$ such that for any $S$-scheme $S^{\prime}$, the pullback endomorphisms $g^{\prime}$ and $h^{\prime}$ of $A^{\prime}=A_{/ S^{\prime}}$ coincide if and only if $S^{\prime} \rightarrow S$ factors through $U$.

Proof. This is essentially [DR, II, 1.14], except that we allow $A$ to be an algebraic space rather than a scheme. The proof carries over to this more general situation. 
By étale descent, Lemma B.2.7 is valid with $S$ and $S^{\prime}$ taken to be algebraic spaces.

B.3. Reduction-type and formal canonical subgroups. Let $k$ be a nonarchimedean field with valuation ring $R$. If $k$ is not discretely-valued then there is no theory of Néron models over $R$ for abelian varieties over $k$. However, in the 1-dimensional case we can easily push through some aspects of the theory. We shall explain how this works, and then link it up with canonical subgroups of 1-parameter commutative formal groups when the reduction type is "not too supersingular."

The starting point is:

Lemma B.3.1 (Faltings). The functor $A \rightsquigarrow k \otimes_{R} A$ from semi-abelian schemes over $R$ to semi-abelian varieties over $k$ is fully faithful.

Proof. Since $R$ is a normal ring, this is a special case of [F, $\S 2$, Lemma 1].

If $E_{1}$ and $E_{2}$ are generalized elliptic curves over Spec $R$ and $c\left(E_{1}\right)$ and $c\left(E_{2}\right)$ are the contractions away from fibral identity components then $c\left(E_{1}\right)^{\mathrm{sm}}$ and $c\left(E_{2}\right)^{\mathrm{sm}}$ are semi-abelian schemes over $\operatorname{Spec} R$. Thus, for any isomorphism $\varphi_{k}$ between the generic fibers of $E_{1}$ and $E_{2}$ there is a unique isomorphism $\varphi: c\left(E_{1}\right)^{\mathrm{sm}} \simeq c\left(E_{2}\right)^{\mathrm{sm}}$ extending the induced map $c\left(\varphi_{k}\right)^{\mathrm{sm}}$ between smooth loci on "contracted" generic fibers. In particular, if $E_{k}$ is an elliptic curve over Spec $k$ then for any generalized elliptic curve $E$ over Spec $R$ with generic fiber $E_{k}$, the property that $E$ has smooth (resp. non-smooth) closed fiber is independent of $E$; if $E_{k}$ is not smooth then $E$ must have non-smooth closed fiber. When there exists a generalized elliptic curve $E$ over Spec $R$ with generic fiber $E_{k}$, we shall say that $E_{k}$ has semi-stable reduction over $R$. Since $c(E)^{\mathrm{sm}}$ is canonical in such cases, the concepts of good reduction and toric reduction are well-defined for those generalized elliptic curves over Spec $k$ that have semi-stable reduction over $R$. In the case of good reduction and positive residue characteristic we may moreover distinguish the cases of ordinary reduction and supersingular reduction. The properties of toric, ordinary, and supersingular reduction for semi-stable generalized elliptic curves over Spec $k$ are unaffected by arbitrary analytic extension on $k$.

Theorem B.3.2. For any generalized elliptic curve $E_{k}$ over $\operatorname{Spec} k$, there exists a finite separable extension $k^{\prime} / k$ (with valuation ring $R^{\prime}$ ) such that $E_{k^{\prime}}$ has semistable reduction over $R^{\prime}$.

Proof. First assume that $E_{k}$ is smooth. Choose an integer $N \geq 5$ with $N \in R^{\times}$, so the proper moduli stack $\mathscr{M}_{\Gamma_{1}(N) / R}$ is a scheme [C2, Thm. 3.2.7, Thm. 4.2.1(2)]. By the valuative criterion for properness, it suffices to pick a finite separable extension $k^{\prime} / k$ that splits the finite étale $N$-torsion $E_{k}[N]$ because $E_{k^{\prime}}$ then admits a $\Gamma_{1}(N)$-structure (so we get a $k^{\prime}$-point of $\mathscr{M}_{\Gamma_{1}(N)}$, and it extends to an $R^{\prime}$-point). 
Now assume $E_{k}$ is non-smooth, so by Lemma B.3.1 it is an fppf form of a standard $n$-gon for some $n \geq 1$. Since the automorphism functor for the standard $n$-gon $C_{n}$ as a generalized elliptic curve over $\operatorname{Spec} \mathbf{Z}$ is represented by a finite semi-direct product $\mathbf{Z}$-group $G_{n}=\mu_{n} \rtimes\langle$ inv $\rangle$ (where "inv" denotes the unique involution extending inversion on the smooth locus), the set of isomorphism classes of $f p p f$-forms of $C_{n}$ over a scheme $S$ is classified (functorially in $S$ ) by the pointed cohomology set $\check{\mathrm{H}}_{\mathrm{fppf}}^{1}\left(S, G_{n}\right)$. By the usual procedure in non-abelian cohomology, there is an exact sequence of pointed sets

$$
\check{\mathrm{H}}_{\mathrm{fppf}}^{1}\left(S, \mu_{n}\right) \rightarrow \check{\mathrm{H}}_{\mathrm{fppf}}^{1}\left(S, G_{n}\right) \rightarrow \check{\mathrm{H}}_{\mathrm{fppf}}^{1}(S, \mathbf{Z} / 2 \mathbf{Z})
$$

and this is functorial in $S$. Since the final term coincides with the analogous étale cohomology set, for any $c \in \check{\mathrm{H}}_{\mathrm{fppf}}^{1}\left(S, G_{n}\right)$ there is a (finite) étale cover $S^{\prime} \rightarrow S$ such that the restriction $\left.c\right|_{S^{\prime}}$ comes from $\check{\mathrm{H}}_{\mathrm{fppf}}^{1}\left(S^{\prime}, \mu_{n}\right)$.

In case $S=\operatorname{Spec}(B)$ is local, Kummer theory functorially identifies $\check{\mathrm{H}}_{\mathrm{fppf}}^{1}\left(S, \mu_{n}\right)$ with $B^{\times} /\left(B^{\times}\right)^{n}$. Thus, in our setting we may replace $B=k$ with a finite separable extension so that $E_{k}$ is classified by an element coming from $k^{\times} /\left(k^{\times}\right)^{n}$, and hence it extends to a generalized elliptic curve over $k$ if this class comes from $R^{\times} /\left(R^{\times}\right)^{n}$. Our problem therefore reduces to showing that for any element $c \in k^{\times} /\left(k^{\times}\right)^{n}$ there is a finite separable extension $k^{\prime}$ of $k$ such that the image of $c$ in $k^{\prime \times} /\left(k^{\prime \times}\right)^{n}$ is in the image of $R^{\prime \times} /\left(R^{\prime \times}\right)^{n}$. This says that $|c| \in\left|k^{\times}\right|$is an $n$th power in $\left|k^{\prime \times}\right|$. Thus, it suffices to prove that $\left|k_{\text {sep }}^{\times}\right|$is a divisible group. But $k_{\text {sep }}$ is dense in an algebraic closure $\bar{k}$ of $k$ [BGR, 3.4.1/6], and so $\left|k_{\text {sep }}^{\times}\right|$coincides with the divisible group $\left|\bar{k}^{\times}\right|$

By this "semi-stable reduction theorem," in the obvious manner we can define the notions of potentially good and potentially toric reduction, and in the potentially good case with positive residue characteristic we have the dichotomy of potentially ordinary and potentially supersingular reduction. When $E_{k^{\prime}}$ extends to a generalized elliptic curve $E^{\prime}$ over $R^{\prime}$ then the preceding considerations show that the semi-abelian $R^{\prime}$-group $c\left(E^{\prime}\right)^{\mathrm{sm}}$ is independent of $E^{\prime}$ and is functorial in $E_{k}$. The formal completion of $c\left(E^{\prime}\right)^{\mathrm{sm}}$ along its identity section will be denoted $\widehat{E}^{\prime}$; this formal group is intrinsic to $E_{k}$ and $k^{\prime} / k$, and it has underlying pointed formal scheme of the form $\operatorname{Spf} R^{\prime} \llbracket X \rrbracket$ with the $X$-adic topology. The formation of this formal group commutes with any further analytic extension on $k^{\prime}$.

Now assume that $k$ is an analytic extension field of $\mathbf{Q}_{p}$, which is to say that $R$ has mixed characteristic $(0, p)$. We normalize the absolute value on $k$ so that $|p|=1 / p$. Consider a generalized elliptic curve $E_{k}$ over Spec $k$ and a finite separable extension $k^{\prime} / k$ such that $E_{k^{\prime}}$ has semi-stable reduction, say with $E^{\prime}$ a generalized elliptic curve over $R^{\prime}$ having generic fiber $E_{k^{\prime}}$. The formal group $\widehat{E}^{\prime}$ of $E^{\prime}$ over $R^{\prime}$ coincides with that of $c\left(E^{\prime}\right)^{\mathrm{sm}}$ and so it is canonically attached to $E_{k}$ (and the choice of $k^{\prime} / k$ ). This is a smooth 1-parameter commutative formal 
over $R^{\prime}$. Since $[p]=V \circ F$ over $R^{\prime} / p R^{\prime}$ [SGA3, VII $\left.\mathrm{A}, \S 4.2-4.3\right]$, if we choose a formal parameter $X$ then we may (non-uniquely) write

$$
[p]^{*}(X)=p X+a X^{p}+X^{2 p} h\left(X^{p}\right)+p X^{2} f(X)
$$

with power series $h$ and $f$ over $R^{\prime}$, so the reduction $\bar{a} \in R^{\prime} / p R^{\prime}$ is independent of $X$ up to $\left(R^{\prime} / p R^{\prime}\right)^{\times}$-multiple. More conceptually, $\bar{a}$ generates the annihilator ideal for the cokernel of $\operatorname{Lie}\left(V_{\widehat{E}^{\prime} \bmod p}\right)$ and $a \in R^{\prime}$ is a lift of $\bar{a}$. Hence, the following definition is intrinsic to $E_{k}$ :

Definition B.3.3. The Hasse invariant of $E_{k}$ is $h\left(E_{k}\right)=\max (|a|, 1 / p) \in[1 / p, 1]$ for any $a \in R^{\prime}$ lifting $\bar{a}$ as above.

Remark B.3.4. By [KM, 12.4.4] and the Serre-Tate theorem relating the infinitesimal deformation theory of an abelian scheme and its $p$-divisible group, if $E_{k}$ is an elliptic curve over $k$ with supersingular reduction $E_{0}$ then we can interpret $h\left(E_{k}\right)$ as the radius of a certain subdisc containing $E_{k}$ as a point on the Berthelot generic fiber (in the sense of Theorem 3.1.5) of the universal deformation ring of $E_{0}$ over $R$.

The reduction of $\widehat{E}^{\prime}$ over the residue field $\widetilde{k}^{\prime}=R^{\prime} / \mathfrak{m}_{R^{\prime}}$ is the identity component of the $p$-divisible group of $c\left(E^{\prime}\right)_{\widetilde{k}^{\prime}}^{\mathrm{sm}}$, so it has height 1 (resp. height 2) when the reduction is a torus or ordinary (resp. supersingular). That is, $h\left(E_{k}\right)=1$ (resp. $\left.h\left(E_{k}\right)<1\right)$ if and only if the reduction type for $E_{k}$ is potentially ordinary or potentially toric (resp. potentially supersingular). Making $h\left(E_{k}\right)$ closer to $1 / p$ makes the reduction-type of $E_{k}$ be "more supersingular".

Suppose that $E_{k}$ is a generalized elliptic curve over Spec $k$. Let $k^{\prime} / k$ be a finite separable extension such that $E_{k^{\prime}}$ has semi-stable reduction, say with $E_{k^{\prime}}$ extending to a generalized elliptic curve $E^{\prime}$ over $\operatorname{Spec} R^{\prime}$, so the formal group $\widehat{E}^{\prime}$ over $R^{\prime}$ is intrinsic to $E_{k}$ and the choice of $k^{\prime} / k$.

Definition B.3.5. The size of a point $x$ of $\widehat{E}^{\prime}$ with values in the maximal ideal of an analytic extension of $k^{\prime}$ is the common absolute value $|X(x)|$ for any formal parameter $X$ of $\widehat{E}^{\prime}$. This is denoted $\operatorname{size}(x)$.

It is clear that the notion of size is Galois-invariant with respect to $k$, and so in particular it is intrinsic to points of $E_{k}$ that extend to points of $\widehat{E}^{\prime}$. The $p$-torsion $\widehat{E}^{\prime}[p]$ is a finite flat $R^{\prime}$-group contained in the quasi-finite flat $R^{\prime}$-group $E^{\prime}[p]$, so $\widehat{E^{\prime}}[p]_{k^{\prime}}$ is a finite $k^{\prime}$-subgroup of $E_{k^{\prime}}^{\prime}[p]=E_{k^{\prime}}[p]$. Obviously $\widehat{E}^{\prime}[p]$ has order $p$ (resp. order $p^{2}$ ) if and only if $h\left(E_{k}\right)=1$ (resp. $h\left(E_{k}\right)<1$ ). Now assume $h\left(E_{k}\right)>p^{-p /(p+1)}$. By using a choice of formal parameter $X$ that linearizes the action of $\mu_{p-1}\left(\mathbf{Z}_{p}\right)$ on the formal group, it is proved in [Lu] (also see [Kz, 3.10.7(1)]) that there is a unique finite flat $R^{\prime}$-subgroup $G_{R^{\prime}}$ in $\widehat{E}^{\prime}[p]$ of order $p$ such that its nontrivial geometric points over $k^{\prime}$ have size $\left(p h\left(E_{k}\right)\right)^{-1 /(p-1)} \in$ 
$\left[p^{-1 /(p-1)}, p^{-1 /\left(p^{2}-1\right)}\right)$, and if $h\left(E_{k}\right)<1$ (so $\left.G_{R^{\prime}} \neq \widehat{E}^{\prime}[p]\right)$ then the other $p^{2}-p$ geometric points of $\widehat{E}^{\prime}[p]$ over $k^{\prime}$ have strictly larger size (in fact, size $h\left(E_{k}\right)^{1 /\left(p^{2}-p\right)}$ that is larger than $\left.p^{-1 /\left(p^{2}-1\right)}\right)$. The subgroup $G_{R^{\prime}}$ is the canonical subgroup of $\widehat{E}^{\prime}[p]$ (or of $\widehat{E}^{\prime}$ ). By construction, the canonical subgroup is compatible with extension on $k^{\prime}$. Hence, we can descend its generic fiber by using étale descent:

Definition B.3.6. Let $E_{k}$ be a generalized elliptic curve over Spec $k$, and assume that $h\left(E_{k}\right)>p^{-p /(p+1)}$. The canonical subgroup $G \subseteq E_{k}^{\mathrm{sm}}$ is the unique étale descent of the generic fiber of the canonical subgroup $G_{R^{\prime}}$ in $\widehat{E}^{\prime}$ for any finite separable $k^{\prime} / k$ as above.

By the definition, the formation of the canonical subgroup in $E_{k}^{\mathrm{sm}}$ commutes with arbitrary analytic extension on $k$. If $h\left(E_{k}\right) \leq p^{-p /(p+1)}$ then all $p^{2}-1$ nonzero geometric points in $\widehat{E}^{\prime}[p]$ have the same size (namely, $p^{-1 /\left(p^{2}-1\right)}$ ) and so there is no good notion of canonical subgroup for such $E_{k}$.

\section{REFERENCES}

[AM] A. Abbes, A. Mokrane, Sous-groupes canoniques et cycles évanescents p-adiques pour les variétes abéliennes, Publ. Math. IHES, 99 (2004), pp. 117-162.

[AG] F. Andreatta, C. Gasbarri, The canonical subgroup for families of abelian varieties, preprint, 2005.

[BKKN] R. Berger et. al., Differentialrechnung in der analytischen Geometrie, Lecture Notes in Math 38, Springer-Verlag, New York, 1967.

[Ber1] V. Berkovich, Spectral theory and analytic geometry over non-Archimedean fields, Mathematical Surveys and Monographs, vol. 33, American Mathematical Society, 1990.

[Ber2] V. Berkovich, Local properties of morphisms, unpublished notes.

[BG] S. Bosch, U. Görtz, Coherent modules and their descent on relative rigid spaces, J. Reine Angew. Math., 495 (1998), pp. 119-134.

[BGR] S. Bosch, U. Günzter, R. Remmert, Non-Archimedean analysis, Springer-Verlag, 1984.

[BL1] S. Bosch, W. Lütkebohmert, Formal and rigid geometry I, Math. Annalen, 295 (1993), pp. 291-317.

[BL2] S. Bosch, W. Lütkebohmert, Formal and rigid geometry II, Math. Annalen, 296 (1993), pp. 403-429.

[BLR1] S. Bosch, W. Lütkebohmert, M. Raynaud, Néron Models, Springer-Verlag, 1990.

[BLR2] S. Bosch, W. Lütkebohmert, M. Raynaud, Formal and rigid geometry IV. The reduced fiber theorem, Inventiones Math., 119 (1995), pp. 361-398.

[BT] K. Buzzard, R. Taylor, Companion forms and weight-1 forms, Annals of Math. 149 (1999), pp. 905-919.

[Bu] K. Buzzard, Analytic continuation of overconvergent eigenforms, Journal of the Amer. Math. Soc. 16 (2003), pp. 29-55.

[CF] C-L. Chai, G. Faltings, Degeneration of abelian varieties, Springer-Verlag, 1990.

[C1] B. Conrad, Irreducible components of rigid spaces, Annales de L'institut Fourier, 49 (1999), pp. 473-541.

[C2] B. Conrad, Arithmetic moduli of generalized elliptic curves, to appear in Journal of the Inst. of Math. of Jussieu.

[C3] B. Conrad, Relative ampleness in rigid-analytic geometry, to appear in Annales de L'institut Fourier. 
[C4] B. Conrad, Higher-level canonical subgroups in abelian varieties, in preparation, 2005.

[DM] P. Deligne, D. Mumford, The irreducibility of the space of curves of given genus, Publ. Math. IHES, 36 (1969), pp. 75-109.

[DR] P. Deligne, M. Rapoport, Les schémas de modules des courbes elliptiques in Modular Functions of One Variable II, Springer Lecture Notes in Mathematics 349 (1973), pp. 143-316.

[EGA] J. Dieudonné, A. Grothendieck, Éléments de géométrie algébrique, Publ. Math. IHES, 4, 8, 11, 17, 20, 24, 28, 32, (1960-7).

[F] G. Faltings, "Finiteness theorems for abelian varieties over number fields," Ch. II in Arithmetic geometry (Cornell/Silverman, ed.), Springer-Verlag, 1986.

[GK] E. Goren, P. Kassaei, The canonical subgroup: a "subgroup-free" approach, to appear in Commentarii Mathematicae Helvetici.

[G] F. Gouvea, Arithmetic of p-adic modular forms, Springer Lecture Notes in Mathematics 1304 (1988).

[SGA3] A. Grothendieck, Schémas en groupes I, Springer Lecture Notes in Mathematics 151, Springer-Verlag, New York (1970).

[deJ] A. J. de Jong, Crystalline Dieudonné module theory via formal and rigid geometry, Publ. Math. IHES, 82 (1995), pp. 5-96.

[Kz] N. Katz, p-adic properties of modular schemes and modular forms in Modular Functions of One Variable III, Springer Lecture Notes in Mathematics 350 (1973), pp. 69-190.

[KM] N. Katz, B. Mazur, Arithmetic moduli of elliptic curves, Annals of Mathematics Studies 108, Princetion University Press, 1985.

[K] R. Kiehl, Analytische familien affinoider algebren, S.-B. Heidelberger Akd. Wiss. Math.Natur. Kl. 1968 (1968), pp. 23-49.

[KL] M. Kisin, K.F. Lai, Overconvergent Hilbert modular forms, Amer. Journal of Math. 127 (2005), no. 4, pp. 735-783.

[Liu] Q. Liu, Un contre-exemple au "critére cohomologique d'affinoidicité", C.R. Acad. Sci. Paris Sér. I Math., 307 (1988), no. 2, pp. 83-86.

[Lu] J. Lubin, Finite subgroups and isogenies of one-parameter formal groups, Annals of Math 85 (1967), pp. 296-302.

[L] W. Lütkebohmert, Formal-algebraic and rigid-analytic geometry, Math. Annalen, 286 (1990), pp. 341-371.

[Mat] H. Matsumura, Commutative ring theory, Cambridge Univ. Press, 1986.

[Mum] D. Mumford, Abelian varieties, Oxford University Press, Bombay, 1970.

[Te] M. Temkin, On local properties of non-Archimedean analytic spaces, Math. Annalen, 318 (2000), pp. 585-607.

Brian Conrad

Department of Mathematics

University of Michigan

Ann Arbor, MI 48109, USA

E-mail: bdconrad@umich.edu 